\title{
Dynamic Assessment of Induced Stresses and In-situ Stress Reorientation during Multistage Hydraulic Fracturing in Unconventional Reservoirs
}

\author{
Liliia Reddy
}

Follow this and additional works at: https://researchrepository.wvu.edu/etd

\section{Recommended Citation}

Reddy, Liliia, "Dynamic Assessment of Induced Stresses and In-situ Stress Reorientation during Multistage Hydraulic Fracturing in Unconventional Reservoirs" (2015). Graduate Theses, Dissertations, and Problem Reports. 6487.

https://researchrepository.wvu.edu/etd/6487

This Thesis is protected by copyright and/or related rights. It has been brought to you by the The Research Repository @ WVU with permission from the rights-holder(s). You are free to use this Thesis in any way that is permitted by the copyright and related rights legislation that applies to your use. For other uses you must obtain permission from the rights-holder(s) directly, unless additional rights are indicated by a Creative Commons license in the record and/ or on the work itself. This Thesis has been accepted for inclusion in WVU Graduate Theses, Dissertations, and Problem Reports collection by an authorized administrator of The Research Repository @ WVU. For more information, please contact researchrepository@mail.wvu.edu. 


\title{
Dynamic Assessment of Induced Stresses and In-situ Stress Reorientation during Multistage Hydraulic Fracturing in Unconventional Reservoirs
}

\author{
Liliia Reddy \\ Thesis submitted \\ to the Benjamin M. Statler College of Engineering and Mineral Resources \\ at West Virginia University \\ in partial fulfillment of the requirements for the degree of \\ Master of Science in \\ Petroleum and Natural Gas Engineering \\ Ebrahim Fathi, Ph. D., Committee Chairperson \\ Professor Samuel Ameri \\ Ali Takbiri, Ph. D. \\ Department of Petroleum and Natural Gas Engineering \\ Morgantown, West Virginia \\ 2015
}

Keywords: Multistage Hydraulic Fracturing, Dynamic Stress Assessment, Theory of Critical Distances, Optimum Fracture Spacing

CCopyright 2015 Liliia Reddy 


\begin{abstract}
Dynamic Assessment of Induced Stresses and In-situ Stress Reorientation during Multistage Hydraulic

Fracturing in Unconventional Reservoirs
\end{abstract}

\title{
Liliia Reddy
}

This research has been dedicated to the optimization of multi-stage hydraulic fracturing by providing a more thorough understanding of fracture and reservoir stress behavior. This work was accomplished by the use of a fully coupled fracture propagation simulator (HFWVU2D developed under RPSEA (Grant/Contract No. \#9122-06)) that couples fracture mechanics and fluid dynamics, unlike past studies, which observe only simple interactions. The underlying objective is to eliminate the misappropriated energy caused by inter-fracture stress interaction and stress reorientation surrounding the induced fracture network by assessing stress magnitude as a function of operation conditions.

This study is based upon an in-house numerical simulation model developed for hydraulic fracture propagation referring to the linear elastic fracture mechanics model "LEFM" using finite element method. The elastic response of the 2-D solid medium and the fluid flow within the fracture is coupled to provide a more realistic depiction of these interactions. The magnitude of stress variation and reorientation is calculated in surrounding areas of simultaneous and sequential hydraulic fracturing of a horizontal well for a wide range of hydraulic fracturing propagation regimes for both homogenous and compositional models.

The results clearly show that there is an optimum distance between hydraulic fractures below which, the change (variation) in magnitude and orientation of stresses leads to significant change in fracture geometry and propagation rate. This change has been impacted mainly through mechanical interaction that leads to higher compressive stress concentrations between fractures. The mechanical interaction becomes stronger by increasing the number of fractures or altering the fracture spacing. The effects have also been investigated in a composite reservoir model with different mechanical properties (i.e., Young's modulus and Poisson's ratio), and operational conditions, such as injection rate and volume. Mechanical properties of different layers in a composite reservoir model significantly impact the fracture geometry and propagation rate when the fracture intercepts different layer boundaries. The magnitude of change in stresses and stress reorientation is also quantified in cross sections with respect to the fracture plane as the fracture propagates.

This work provides an advanced understanding of multiple hydraulic fracturing stimulation and dynamics of fracture geometry, propagation rate and stress change in surrounding using our unique fully coupled hydraulic fracturing simulator. Moreover, it provides quantitative analysis of induced stresses and in-situ stress reorientation. The work is important for the optimization of multi-stage hydraulic fracturing in unconventional reservoirs. 


\section{Dedication}

This research work is dedicated to:

My parents for their love, support and continuous encouragement in achieving my goals. I also dedicate this work to my brother for supporting me in every possible way, and all my friends for their words of inspiration in the course of my graduate studies. 


\section{Acknowledgements}

I would also like to thank Professor Samuel Ameri for his continuous guidance and support throughout my Master's Degree at West Virginia University. Special thanks go to my advisor, Dr. Ebrahim Fathi, for

kindly accepting me as his research assistant and for his patience and direction throughout my research, and most of all the encouragement he has given me.

I would like to thank my committee members for generously dedicating their time on reviewing my thesis and giving me valuable recommendations. My gratitude goes to Professor Samuel Ameri and Dr. Ali

Takbiri, who graciously accepted to be a part of my advising committee.

I would like to thank the rest of the faculty at the Department of Petroleum and Natural Gas Engineering at West Virginia University for their expertise, dedication and contribution.

I would like to thank my colleagues from the Department of Petroleum and Natural Gas Engineering, and especially peers from my research group, for their teamwork and encouragement during my graduate studies.

Finally, I would like to thank RPSEA's Funding for this project. RPSEA (www.rpsea.org) is a multipurpose entity whose mission is to provide stewardship in ensuring the focused research, development, and deployment of safe and environmentally responsible technology that can effectively deliver hydrocarbons from domestic resources to the citizens of the United States. 


\section{Table of Contents}

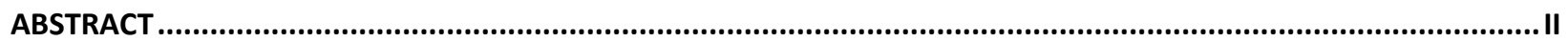

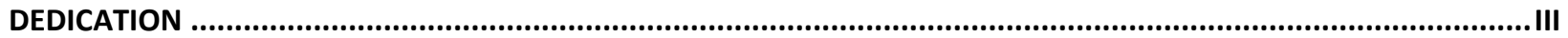

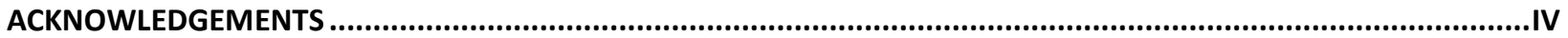

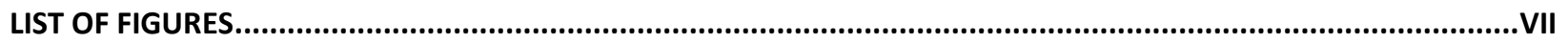

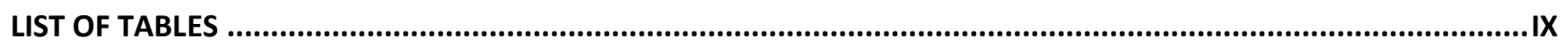

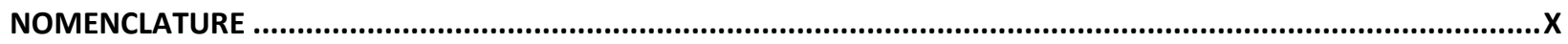

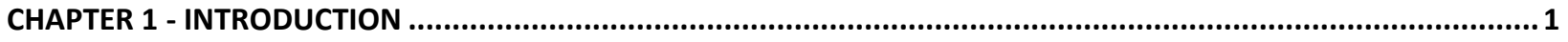

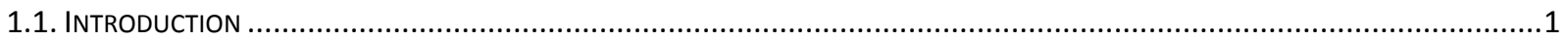

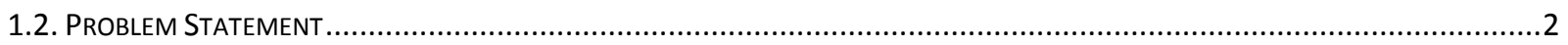

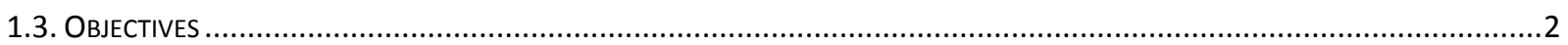

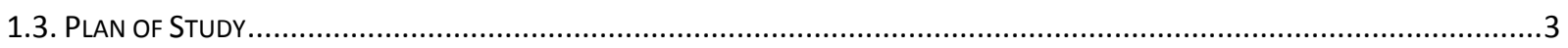

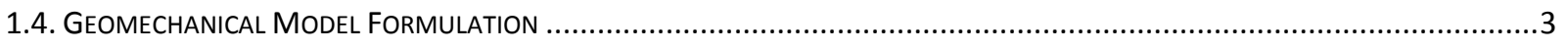

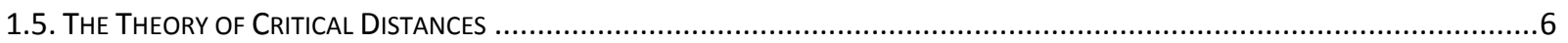

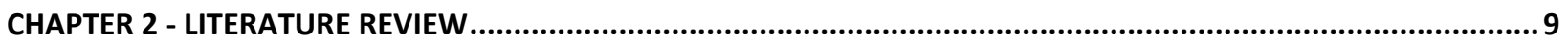

2.1. Application of Theory of Critical Distances in Hydraulic Fracturing .......................................................

2.2. Previous Work in Optimization Fracture Spacing and Sequencing in Horizontal Well Fracturing ......................10

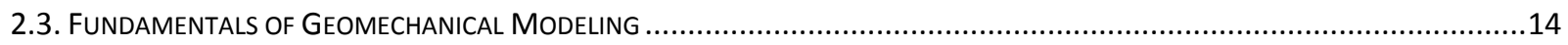

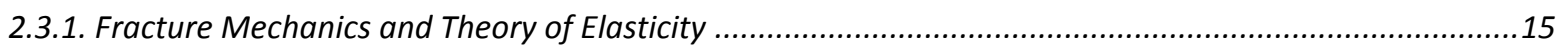

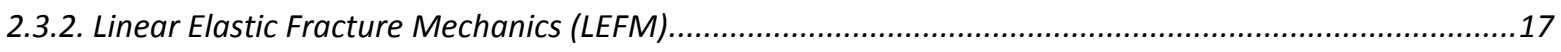

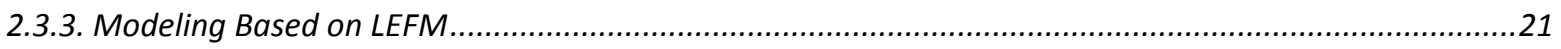

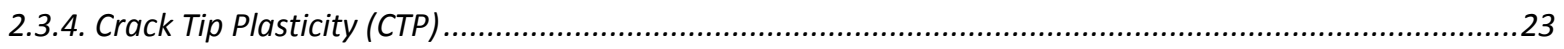

2.4. 2D, PSEUdo-3D, Planar 3D and Fully 3D Hydraulic Fracturing Simulators ................................................26

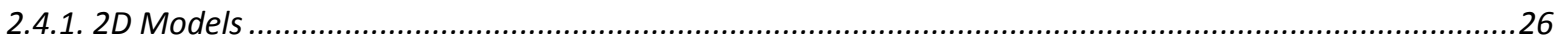

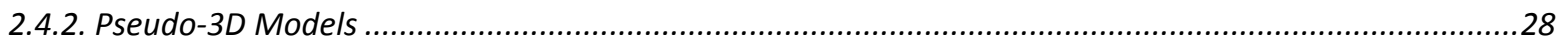

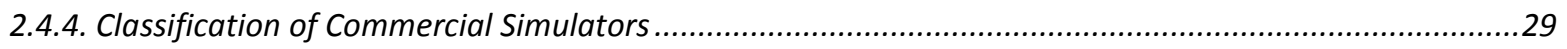

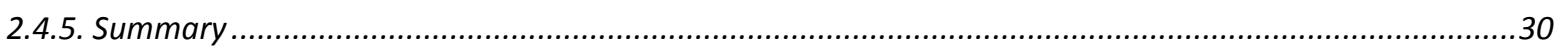

CHAPTER 3 - HFWVU-2D AND MFRAC COMPARISON AND LIMITATIONS ......................................................31

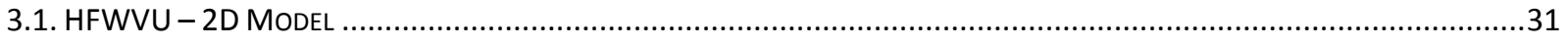

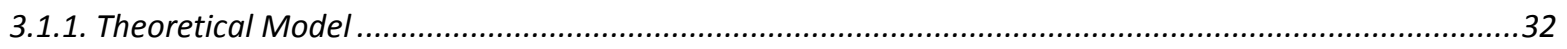

3.1.2. Energy Dissipation Regimes and Asymptotic Solutions...................................................................33

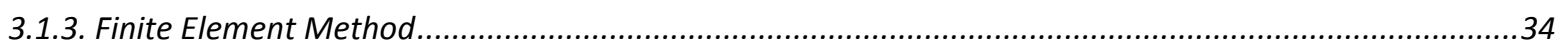

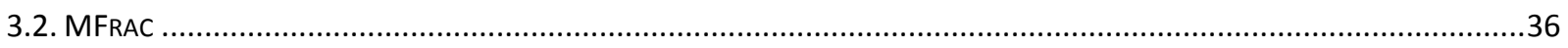

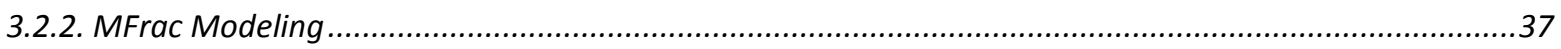

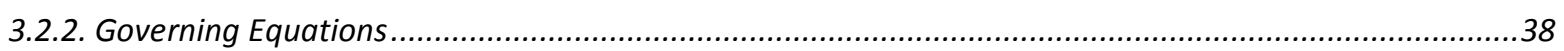

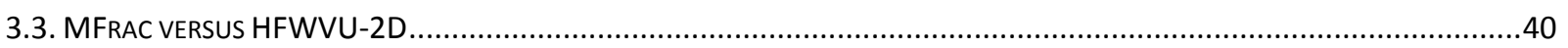

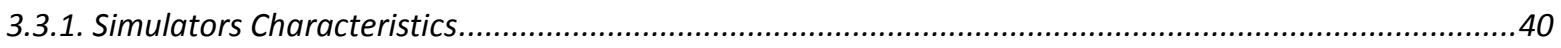

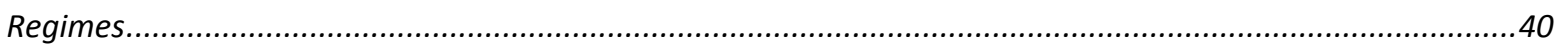

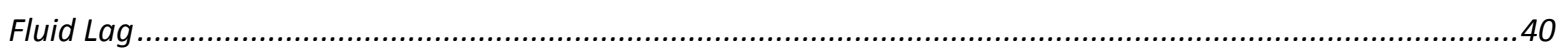

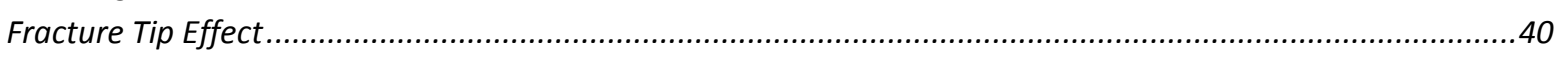




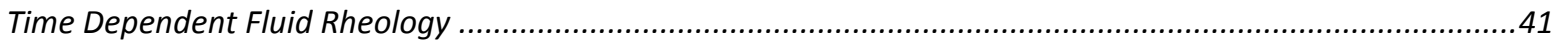

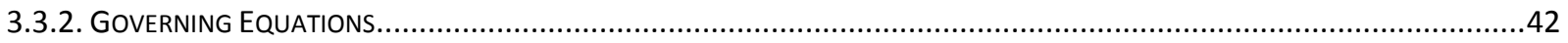

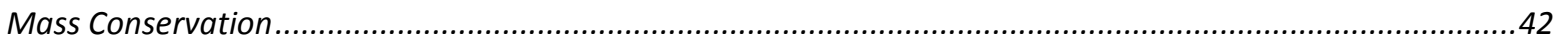

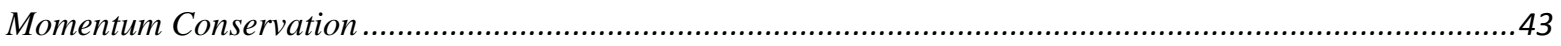

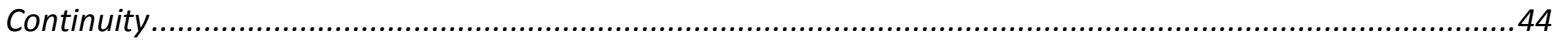

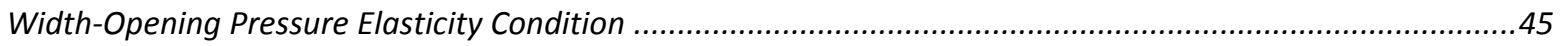

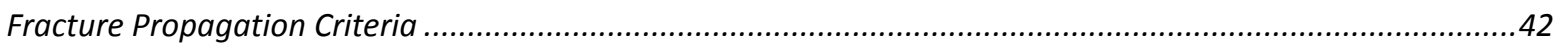

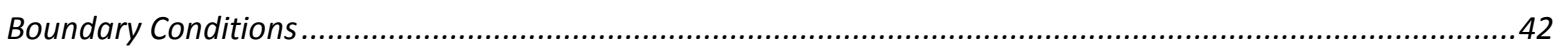

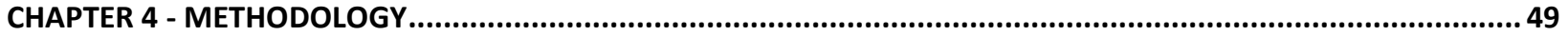

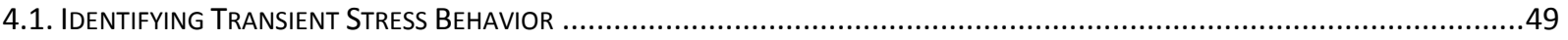

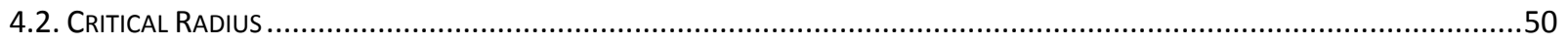

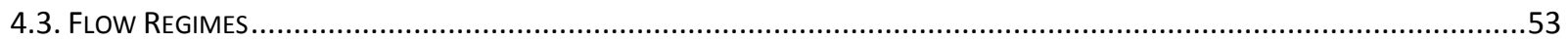

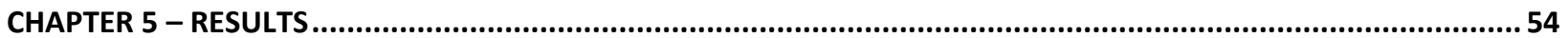

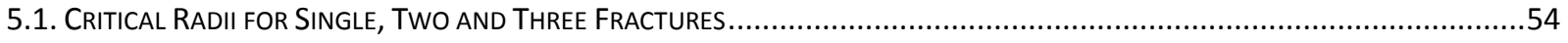

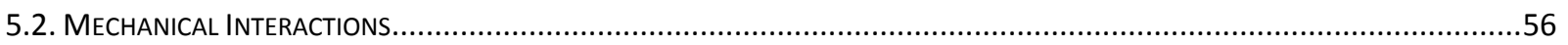

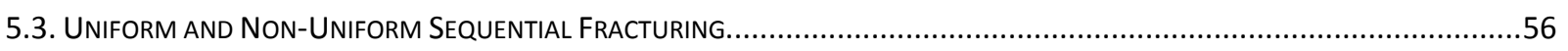

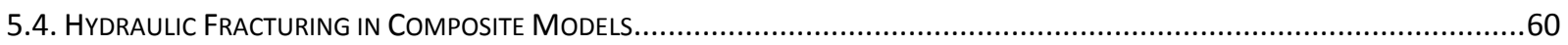

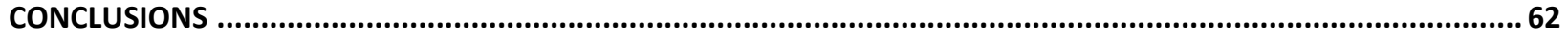

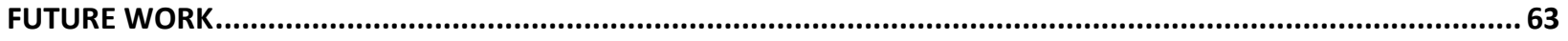

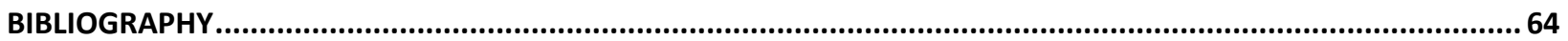




\section{List of Figures}

Figure 1 - (a) Single fracture model with 100 elements on pre-defined fracture path and (b) close view of the fracture area

Figure 2 - Discretization of the solid medium with finite elements in HFWVU ……...............................................4

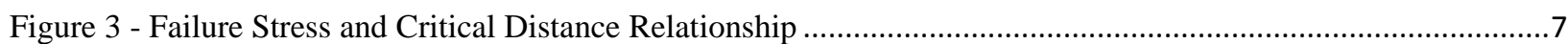

Figure 4 - Tensile stress intensity at a distance beyond the periphery of a circular void. In contrast to 'maximum stress concentration', stress intensity at a constant distance is influenced by the absolute size of the void

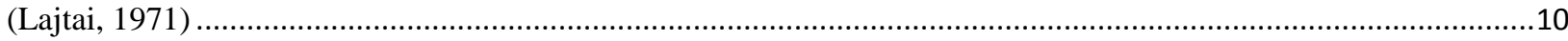

Figure 5 - 3-D model of multiple horizontal fractures in a reservoir with bounding layers ......................................11 Figure 6 - Direction of maximum horizontal stress and angle of stress orientation from multiple transverse fractures

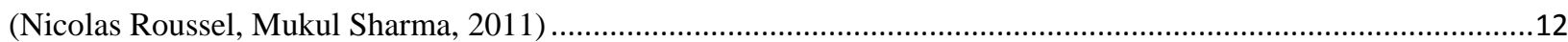

Figure 7 - Maximum horizontal stress reorientation caused by (a) mechanical effects and

(b) poroelastic effects (Nicholas Roussel, Mukul Sharma, 2011)

Figure 8 - Direction of maximum horizontal stress for the case of minimum/maximum stress ratio of 0.99 .

The recommended fracture spacing is 483 feet, where the reorientation of maximum horizontal stress is down to

five degrees (J. Morrill, J. Miskimins, 2012).

Figure 9 - Direction of maximum horizontal stress for net pressure of 3,500 psi. The direction of maximum horizontal stress has reoriented 90 degrees near the fracture. The recommended fracture spacing is 267 feet, where the reorientation of maximum horizontal stress is down to five degrees .............................................................14

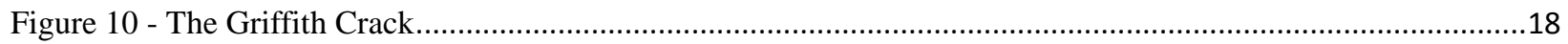

Figure 11 - Coordinate System for Stress Intensity Factor, K .......................................................................19

Figure 12 - Distribution of stresses near the tip of a through-thickness crack in a plate ..........................................20

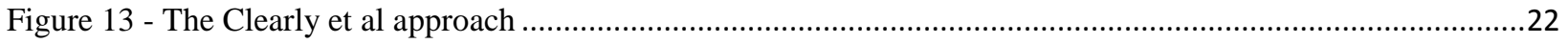

Figure 14 - The Effect of Plastic Yielding on the Stress at a Distance $r$ from the fracture tip..................................24

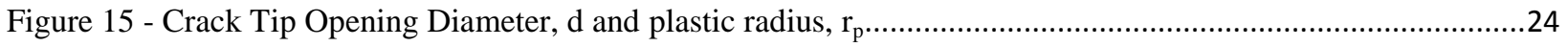

Figure 16 - Dugdale Plastic Zone Strip Model for non-strain hardening solids under plane....................................25

Figure 17 - Fracture geometry for a) PKN, b) GDK and c) Radial Models

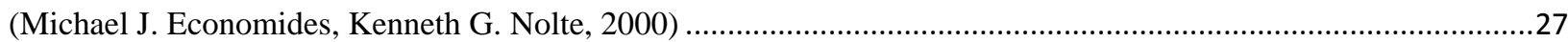

Figure 18 - Hydraulic fracturing regimes in the plane strain model ......................................................................33

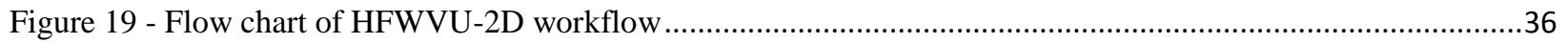

Figure 20 - Fracture tip width reduction due to non-linear elastic effects (Meyer \& Associates, 2015) .....................41

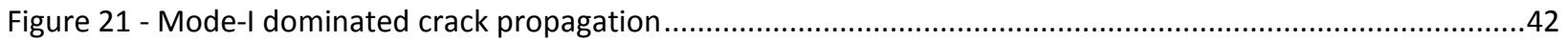

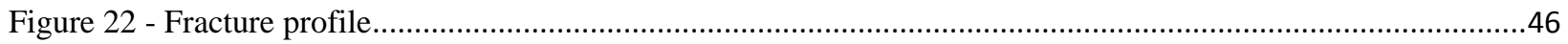

Figure 23 - The transient behavior of maximum principal and Von Mises stress variations surrounding a single

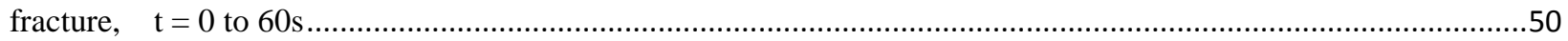


Figure 24 - Maximum Von Mises Stress with relation to the fracture length at distance from the fracture tip and (a) at early simulation time; (b) at late simulation time.

Figure 25 - Maximum Von Mises Stress with relation to the fracture length at distance from the fracture tip and at

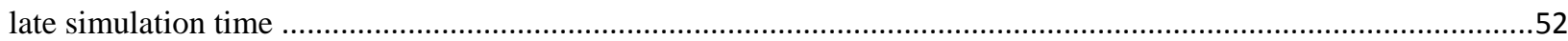

Figure 26 - Points of investigation for static Von Mises Stress Distribution............................................................52

Figure 27 - Von Mises stress along the fracture half-length at equal snapshots in time, $t=60$ s (a) large viscosity case,

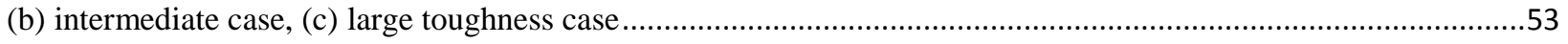

Figure 28 - Critical radius at the fracture tip after 90s of injection...........................................................................54

Figure 29 - Critical Radius at the fracture tip of single, two and three fractures after 10s of injection ....................55

Figure 30 - Development of (a) maximum and (b) minimum principal stresses for three sequential fractures with

time

Figure 31 - Sequential fracture propagation under uniform injection rate and a 5-meter spacing …........................57

Figure 32 - Sequential fracture propagation under non-uniform injection rate (40/60) and a 5-meter spacing ...........57

Figure 33 - Development of fracture half-length geometry of three concurrent fractures at a 5-meter spacing ..........58

Figure 34 - Development of the Von Mises stress surrounding three concurrent fractures at 5 meters spacing .........59

Figure 35 - Display of fracture interaction based upon central fracture ……............................................................59

Figure 36 - Von Mises stress distribution throughout fracture propagation in composite model .............................60

Figure 37 - Fracture geometry due to mechanical interaction in a composite model ................................................61 


\section{List of Tables}

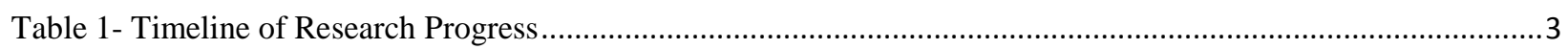

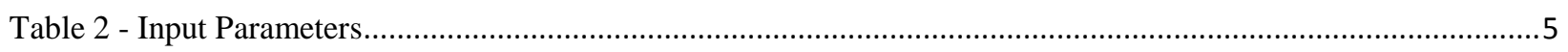

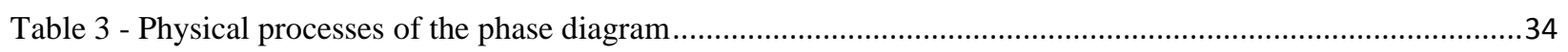

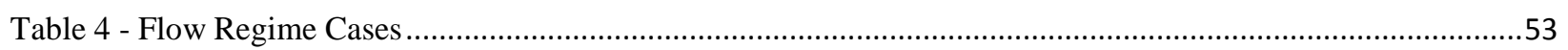

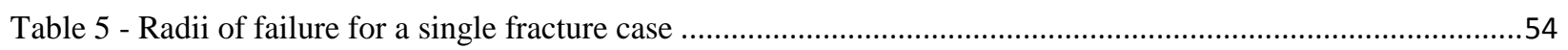

Table 6 - Summary of composite case simulation models .................................................................................60 


\section{Nomenclature}

1. $K_{I}$ stress intensity factor

2. $K_{I C}$ fracture toughness

3. $\nabla \cdot$ divergence operator

4. $\sigma \quad$ stress

5. $b$ gravity

6. D elastic stiffness tensor

7. : double dot product operator

8. $\varepsilon \quad$ strain

9. $u$ node displacement

10. $\nabla$ gradient operator

11. $w \quad$ fracture width

12. $q$ leak-off flux

13. $g$ leak-off term

14. $\mu^{\prime}$ modified viscosity

15. $p_{f} \quad$ fluid pressure

16. $C_{1}^{\prime}$ modified leak-off coefficient

17. $x$ fracture tip arrival time at point $(x)$

18. $\sigma_{0}$ materials related strength

19. $K_{I C}$ plain strain fracture toughness

20. $\sigma_{U T S}$ material Ultimate Tensile Strength

21. $L \quad$ critical distance

22. $\sigma_{d}$ maximum tensile stress

23. $\sigma_{m}$ maximum tensile stress at the boundary

24. $d$ distance along the fracture path

25. $v_{p} \quad$ Poisson's ratio in the pay zone

26. $E_{b} \quad$ Young's moduli of the bounding layers

27. $E_{p} \quad$ Young's moduli of the reservoir

28. $h_{f}$ fracture height

29. $h_{p}$ reservoir height

30. $P$ mechanical force

31. A original cross-sectional area

32. $d \mu \quad$ change of displacement

33. $E$ tensile modulus

34. $G$ shear modulus

35. $a_{0}$ equilibrium atomic distance 


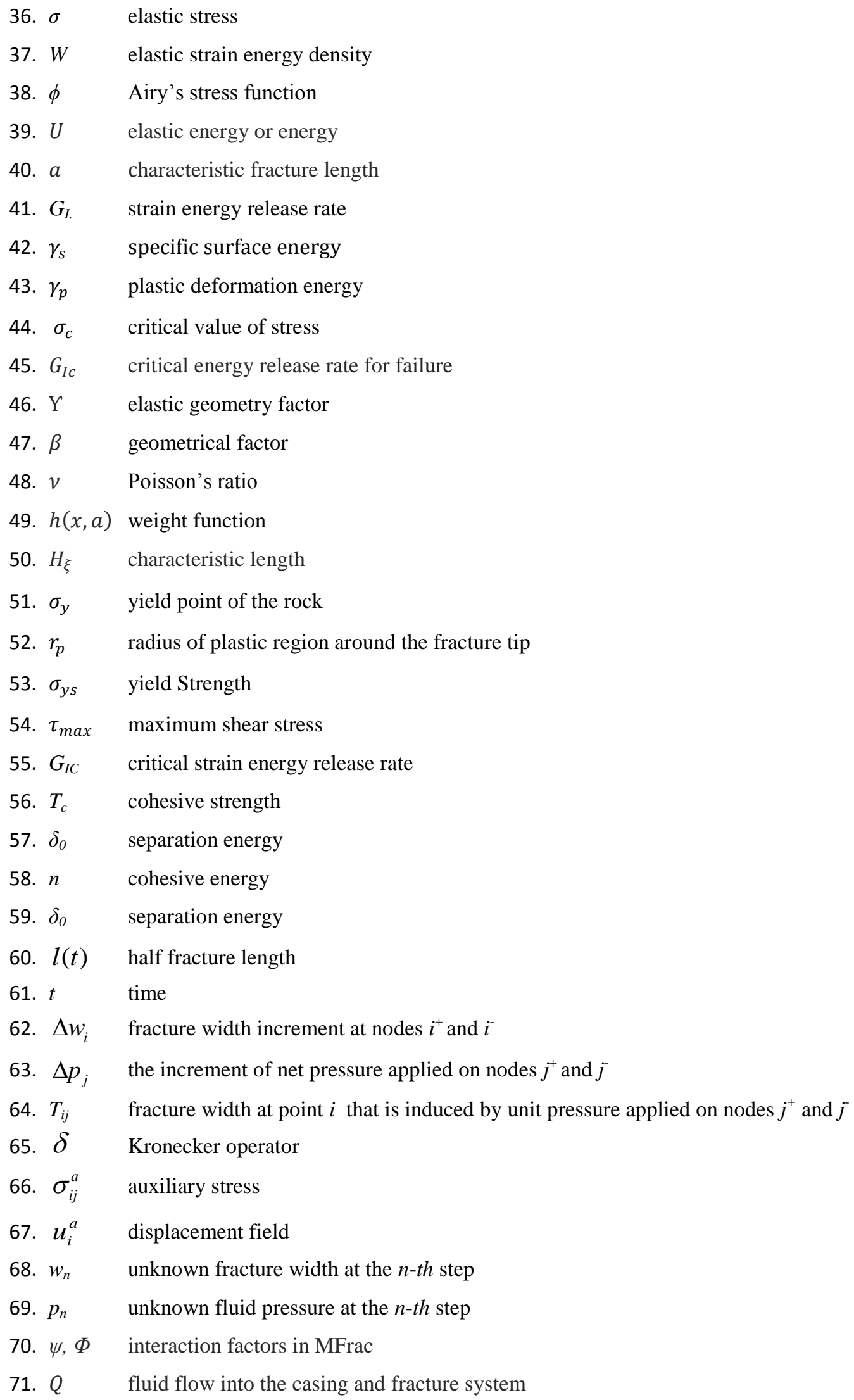


72. $Q_{t}$ fluid flow with consideration of wellbore storage and recirculation

73. $W$ fracture width in MFrac

74. $F \quad$ Darcy friction factor

75. Re Reynolds Number

76. $E$ relative wall roughness in MFrac

77. $x$ lateral coordinate along fracture length

78. $y$ coordinate perpendicular to frac face

79. $\mathrm{z}$ coordinate parallel to frac face

80. $\Gamma_{W}$ generalized influence function in MFrac

81. $\Delta P \quad$ net fracture pressure

82. $V_{f} \quad$ volume of slurry in the fracture

83. $V_{l} \quad$ volume of fluid loss to the formation by leak-off

84. $V_{s p} \quad$ spurt loss

85. $Q_{0} \quad$ injection rate

86. $n \quad$ unit normal of the fracture

87. $\sigma_{0} \quad$ confining stress

88. $v_{0}$ leak-off velocity

89. $\Delta p_{R} \quad$ pressure differential carried by the reservoir

90. $v_{c}$ leak-off velocity at closure

91. $F(\theta)$ dimensionless linear time function

92. $F_{L}\left(t, t_{c}\right)$ dimensionless fluid loss time function

93. $x t_{c} \quad$ apparent closure time defined by Nolte (1997)

94. $D$ distance to the point of investigation with the highest probability of failure

95. $\sigma_{V M}$ maximum Von Mises stress at the specific point of investigation

96. $R \quad$ critical radius with high probability of failure 


\section{Chapter 1 - Introduction}

\subsection{Introduction}

Optimization of multistage hydraulic fracturing has an evident impact on well production in unconventional oil and gas reservoirs. The shift towards developing unconventional resources, accordingly, has carried along a number of studies related to multistage hydraulic fracturing, particularly, optimization of a number of fractures and fracture spacing. However, simplified models used for multistage hydraulic fracturing design resulted in significant practical problems including merging hydraulic fracturing stages and reducing the efficiency of the stimulation. Thus, quantitative analysis of the magnitude of the induced stress and in-situ stress reorientation with dynamics of net fluid pressure is of a particular significance.

Hydraulic stimulation first triggered the interest of engineers and scientists a couple of decades ago, when hydraulic fracturing was recognized as an important technology for extracting hydrocarbons. At that time, the inconsistency in results from different hydraulic fracturing simulators was primarily captured by Warpinski, who developed a comparative study of twelve fracture models and acknowledged the discrepancy in results for up to 50\% amongst the selected models (Warpinski et al., 1993). More advanced model alternatives have been developed ever since, offering a design of a complex fracture network in the reservoir, such as simul-frac or zipper frac (Mutalik and Gibson, 2008). When creating better conditions to increase a production rate and deplete a reservoir more effectively, it is important to understand the dynamic assessment of induced stresses and in-situ stress reorientation during multi-stage hydraulic fracturing in order to forecast and avoid stress interference and fracture merging.

Over the years, efforts have been directed towards studying hydraulic fracture reorientation as a result of stress interference (Wright et al., 1995; Roussel and Sharma 2010; Morrill and Miskimins, 2012). However, there are still more concerns needed to be addressed and incorporated in one study to reduce limitations of the complex design. They include identifying stress patterns in a real time for fractures with different sequencing, propagating through various boundary layers, without discounting complex model features. Previous studies in the literature mostly focused on mentioned above aspects in a separate order (Siebrits et al., 1998; Soliman and Adams 2004).

In this work, our in-house numerical simulation model for hydraulic fracture propagation is used to provide a more realistic representation of general stress field characterization in combination with the modified theory of critical distances. The new theory was used, which allows predicting the critical radius around the fracture tip (i.e, highest possibility of failure) for any time step during the stimulation. The theory was examined for a single and multiple fractures, which provides a tool to predict the possibility of fracture merging during simultaneous and sequential hydraulic fracturing.

Chapter 1 serves an introductory part of this research work, offering a geomechanical model formulation and describing the theory of critical distances. Chapter 2 provides an overview of the application of the theory of critical distances in hydraulic fracturing, previous work in optimizing fracture spacing and sequencing in horizontal well fracturing, and fundamentals of geomechanical modeling. A comparison summary between our in-house model HFWVU-2D and commercial software MFrac can be found in Chapter 3. This chapter also includes governing equations and boundary conditions of the simulators, as 
well as a profound analysis of their advantages and limitations. Chapter 4 covers a methodology part of this thesis, identifying transient stress behavior in the reservoir, and further extending a theory of critical distances, applied for defining optimum fracture spacing in a real time. Also, various hydraulic fracturing flow regimes, showing the different impact on magnitude and orientation of stress around hydraulic fractures, are described in this chapter. The results of this research work are displayed in Chapter 5, including critical radii for single, two, and three fractures and optimized fracture spacing; mechanical interactions; uniform and non-uniform sequential fracturing; and hydraulic fracturing in composite models. Conclusions chapter highlights the summary of this research, and also offers recommendations for a future work.

\subsection{Problem Statement}

A comprehensive analysis of stress-field variation needs to be undertaken for a better understanding of the design with multiple transverse fractures. The purpose of this study is to provide a quantitative analysis of induced stresses and in-situ stress reorientation as a function of net fluid pressure on the fracture surface for cases with simultaneous and sequential hydraulic fracturing of a horizontal well for a wide range of hydraulic fracturing propagation regimes for both homogenous and composite reservoir models. As was noticed, fracture geometry is dependent on fracture spacing and a number of fractures (Cheng, 2009; Zhong et al., 2014; Gao et al., 2013). Therefore, our study investigates the effect of mechanical interaction in a homogeneous and composite reservoir models with various mechanical properties and operational conditions. The details of numerical scheme and validation of our in-house simulator with asymptotic solutions can be found in Bao et al., 2014 and 2015. The model has also been used earlier to investigate the impact of proppant distribution in hydraulic fracturing optimization in Marcellus shale by Kong et al., 2015.

\subsection{Objectives}

This research has been dedicated to the optimization of multi-stage hydraulic fracturing by providing a more advanced understanding of fracture geometry and reservoir stress behavior. Models with an assumption of stress remaining static during fracture propagation do not account for a change in local stress and is the main reason of stress interference and fracture merging.

Transient stress development is commonly disregarded during solutions in commercially available software, and it has been an inefficient use of fracturing fluids injected downhole. Applying a fully coupled hydromechanical model with a dynamic assessment of stress behavior is a key solution for a hydraulic fracturing spacing optimization. Investigating in-situ stress changes surrounding single and multiple hydraulic fractures and evaluating and characterizing the stress state that occurs throughout the reservoir, and developing a relationship based upon the stresses so that we can predict the behaviors of propagating fractures helps to optimize multi-stage hydraulic fracturing design and prevent fracture merging.

An underline objective of this thesis is to eliminate the misappropriated energy caused by inter-fracture stress interaction and stress reorientation surrounding the induced fracture network by assessing stress magnitude as a function of operation conditions. This goal is accomplished by applying our fully coupled 
hydromechanical finite element hydraulic fracture model and a theory of critical distances, which was further extended in my study.

\subsection{Plan of Study}

Table 1- Timeline of Research Progress

\begin{tabular}{|c|l|l|l|l|l|l|}
\hline \multirow{2}{*}{ Task } & \multicolumn{3}{|c|}{2014} & \multicolumn{2}{c|}{2015} \\
\cline { 2 - 7 } & Spring & Summer & Fall & Spring & Summer & Fall \\
\hline Coursework & & & & & & \\
\hline Literature Review & & & & & & \\
\hline Developing the Code & & & & & & \\
\hline Pre-Processing Data & & & & & & \\
\hline Processing Data & & & & & & \\
\hline Preliminary Results & & & & & & \\
\hline Extension of TCD & & & & & & \\
\hline
\end{tabular}

\subsection{Geomechanical Model Formulation}

A recently amplified effort directed towards providing a more advanced study of stress behavior and investigating stress profiles around the fracture in real time, requires more emphasis to be made on model features. Our in-house 2-D numerical simulator (HFWVU) fully couples solid medium with fluid flow within the fracture and is capable of providing a realistic representation of stress interference. The simulator is time adaptive and is based on the plain strain PKN model. The coupling method incorporates the following complex physical processes: the non-local relationship between fracture width and net pressure during fracture propagation time; linear dependence of fluid flow on pressure gradient and its non-linear dependence on fracture width; history-dependent fluid leak-off into the formation; and configuration variation of fracture propagation. The dynamics of fluid lag are also captured using our model. The major assumptions of the model include a half-infinite space due to symmetry and elastic response of the solid medium.

The elastic effect is achieved by discretization of the model using finite elements (Figure 1), where the fracture propagates when $\left(K_{I}\right)$, stress intensity factor (SIF), exceeds $\left(K_{I C}\right)$ (fracture toughness) at the fracture tip, and satisfies the following propagation criterion for each time step:

$$
K_{I} \leq\left(1+\varepsilon^{\prime}\right) K_{I C}
$$

The small value of allowable tolerance for SIF $\left(\varepsilon^{\prime}=0.001\right)$ and time adaptive nature of the simulator assures the completely grid independent simulation results (Bao et al., 2015). Computation cost is a major problem in using finite element methods to simulate hydraulic fracturing. In this research work a nodesplit method (Johnson and Carrigan, 2012) and condenstation technique is used to address this issue (Figure 2). 
The elastic response at any point $(x)$ is governed by the equillibrium condition and the constitutive law, written as:

$$
\nabla \cdot \sigma+b=0
$$

and

$$
\sigma(x)=D: \varepsilon(x)
$$

In Equation (2) $(\nabla \cdot)$ is a divergence operator, $(\sigma)$ is stress, and $(b)$ is gravity. $(D)$ in the above equation is known as elastic stiffness tensor, $(:)$ is double dot product operator, and $(\varepsilon)$ is strain.

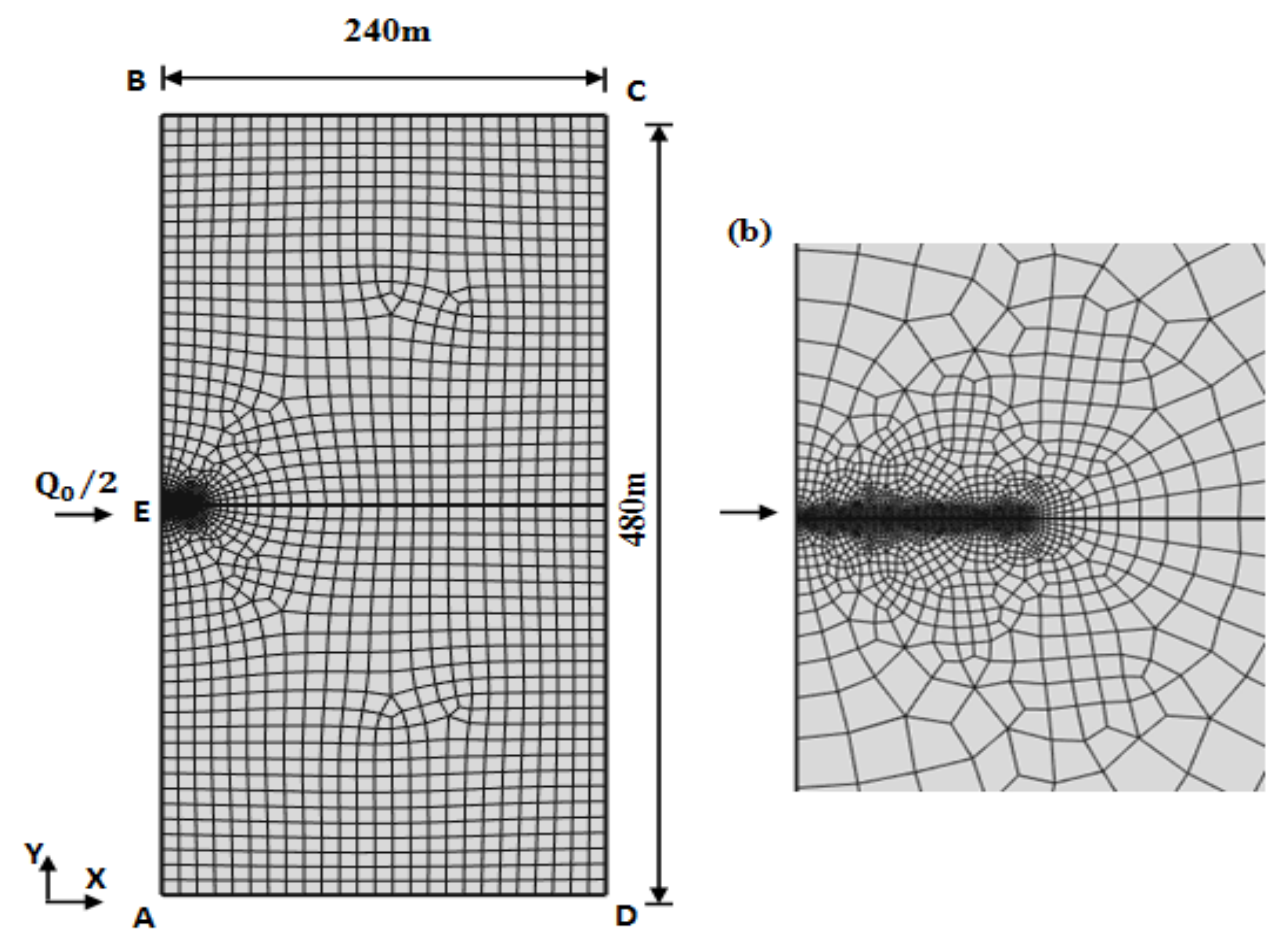

Figure 1 - (a) Single fracture model with 100 elements on pre-defined fracture path and (b) close view of the fracture area

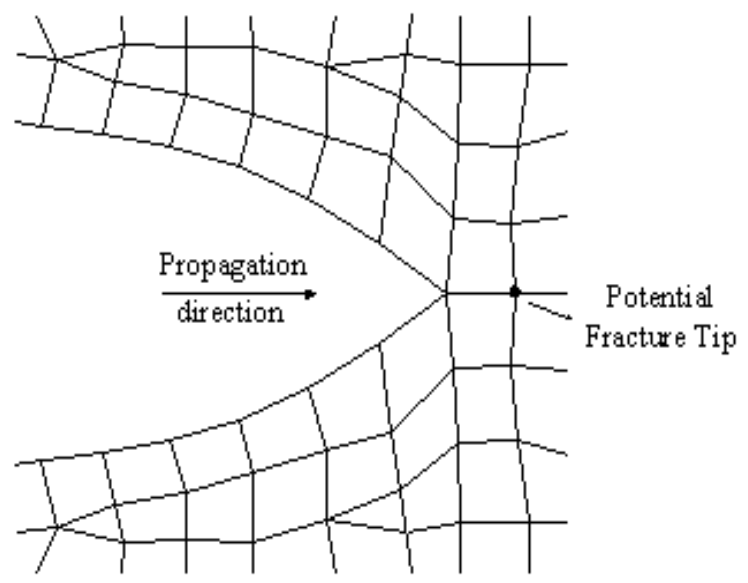

Figure 2 - Discretization of the solid medium with finite elements in HFWVU 
Fracture geometry is computed based on the node displacement $(u)$ :

$$
\varepsilon(x)=[\nabla(u)+(u) \nabla] / 2
$$

Here $(\nabla)$ is known as a gradient operator.

The governing equations in the fluid computation part include mass conservation, fluid flow in the fracture, leak-off and previously discussed fracture propagation Equation (1). The mass conservation for incompressible fluid flow in the fracture has the following expression:

$$
\frac{\partial w}{\partial t}+\frac{\partial q}{\partial x}+g=0
$$

Where $(w)$ represents fracture width, $(q)$ is leak-off flux, and $(g)$ is a leak-off term, which will be explained in Equation (7). The fluid flow in the model is computed based on the lubrication theory, where the fluid flow in the fracture is governed by Poiseuille's Law:

$$
q=-\frac{w^{3}}{\mu^{\prime}} \frac{\partial p_{f}}{\partial x}
$$

Here $\left(\mu^{\prime}\right)$ is modified viscosity $\left(\mu^{\prime}=12 \mu\right)$ and $\left(p_{f}\right)$ is fluid pressure applying on the fracture surface. The fluid leak-off to the medium is characterized by Carter's model:

$$
g(x, t)=\frac{C_{1}^{\prime}}{\sqrt{t-t_{0}(x)}}
$$

In Equation (7), $\left(C_{1}^{\prime}\right)$ is modified leak-off coefficient $\left(C_{1}^{\prime}=12 C_{1}\right)$ and $(x)$ is fracture tip arrival time at point $(x)$.

The model verification was accomplished by comparing numerically computed mass conservation, fluid flow and leak-off values with semi-analytical solutions (Adachi, J. and Detournay E., 2008).

The input parameters used for running various simulation cases are summarized in Table 2.

Table 2 - Input Parameters

\begin{tabular}{|lll|}
\hline Parameter & Value & Unit \\
\hline Poisson's Ratio & 0.209 & $\mathrm{GPa}$ \\
Young's Modulus & 17 & $\mathrm{~m}^{2} / \mathrm{s}$ \\
Injection Rate & 0.0005 & $\mathrm{~m} / \sqrt{\mathrm{s}}$ \\
Leak-Off Rate & $1.47 \mathrm{e}-5$ & $\mathrm{~m}$ \\
Fracture Spacing & 5 & $\mathrm{KPa} / \mathrm{s}$ \\
Dynamic Viscosity & $9 \mathrm{e}-7$ & $\mathrm{~K}$ \\
\hline
\end{tabular}




\subsection{The Theory of Critical Distances}

The theory of critical distances (TCD) has been established as a method for predicting fatigue and fracture in a wide range of materials and components, and it is valid both for research and engineering applications. The TCD can be described as a set of theories that introduced a critical distance, L, to measure the local stress ahead of the stress concentration feature, also known as a stress peak. The TCD includes four methods of application, such as the Point Method (PM), the Line Method (LM), the Area Method (AM), and the Volume Method (VM) (Louks et al., 2014).

The history of studies goes back to 1930s when critical distance analysis was first introduced, and by 1950s, Neuber has first published a method that replicates the concept of the TCD LM. Neuber averaged the elastic stress ahead of the highest stress accumulation point over the distance. A few years later, Peterson invented similar to the TCD PM, however, a more simplified method. Peterson developed a theory of a component failure when the elastic stress at a material dependent distance from the stress accumulation point reached a critical value. Neuber and Peterson focused their research on investigating the fatigue failure of notched metallic components. During their developmental study related to critical distance methods, both researchers experienced the following issues:

1. Both Neuber and Peterson selected an empirical approach in measuring a material dependent length, fitting predictions to data. Peterson's idea was based on defining the critical distance in correlation to the grain size. However, he faced a few challenges during developmental process of this technique.

2. Obtaining correct stress-distance plots in real components was not manageable to achieve. With a variety of solutions that Neuber suggested for standard notch geometries, they only produced approximations when applied to real components.

In 1974, Whitney and Nuismer established a relationship between Continuum Mechanics and Linear Elastic Fracture Mechanics (LEFM), and they described the critical distance as a function of fracture toughness, $K_{I C}$. Moreover, due to the lack of available information about previous work in the theory of critical distance, they reproduced the theories identical to the LM and PM, but named them differently (Whitney and Nuismer, 1974).

A material dependent length $L$ was defined with the following equation:

$$
L=\frac{1}{\pi}\left(\frac{K_{I C}}{\sigma_{0}}\right)
$$

where $\left(\sigma_{0}\right)$ is the materials related strength and $\left(K_{I C}\right)$ is the plain strain fracture toughness. Both parameters from this equation, $\sigma_{0}$ and $K_{I C}$, have to be determined from experimental measurements for each material. When material related strength $\sigma_{0}$ becomes greater than the material Ultimate Tensile Strength (UTS), TCD predicts material failure with a large error.

The first book about TCD was published in 2007 by David Taylor "The Theories of Critical Distances, $a$ New Perspective in Fracture Mechanics", and it was based on the previous work in this area of study. In his book, Taylor first mentioned that the stress and strain information at the notch surface, where the stress peak is located, also known as "hot spot", is not sufficient to predict failure arising from a stress 
concentrator apex. In his book, Taylor elaborated on the influence of the change in local stress on fracture initiation and propagation process, and he introduced a non-local solution approach for predicting cracktype material failures. He assured that the stress field away from the crack notch also carries important aspects, such as the gradient of stress or the absolute volume of material that is experiencing high stress, and it could affect crack propagation.

Taylor hypothesized that some very brittle engineering ceramics have a characteristic strength $\sigma_{0}$ equal to the material UTS, $\sigma_{U T S}$. Nevertheless, in 2008 Taylor collaborated with Susmel, and they have proven that the assumption of $\sigma_{0}=\sigma_{U T S}$ was incorrect and not able to deliver accurate results, unlike the original TCD. A special concern was of investigating the failure of those materials that are capable to experience limited plasticity in the vicinity of the stress raiser peak. Previous studies have been applied mostly for solving linear-elastic problems, where any non-linear material behavior due to plasticity or damage is restricted to a small process zone. TCD has been used to predict brittle fatigue and failure in all types of materials: metals, polymers, ceramics and composites. Comparing to the earlier studies in this area, Taylor's work has shown this approach to be applicable for problems involving plastic behavior, for example low and medium-cycle fatigue and the static fracture of tough metallic materials (Taylor, 2007).

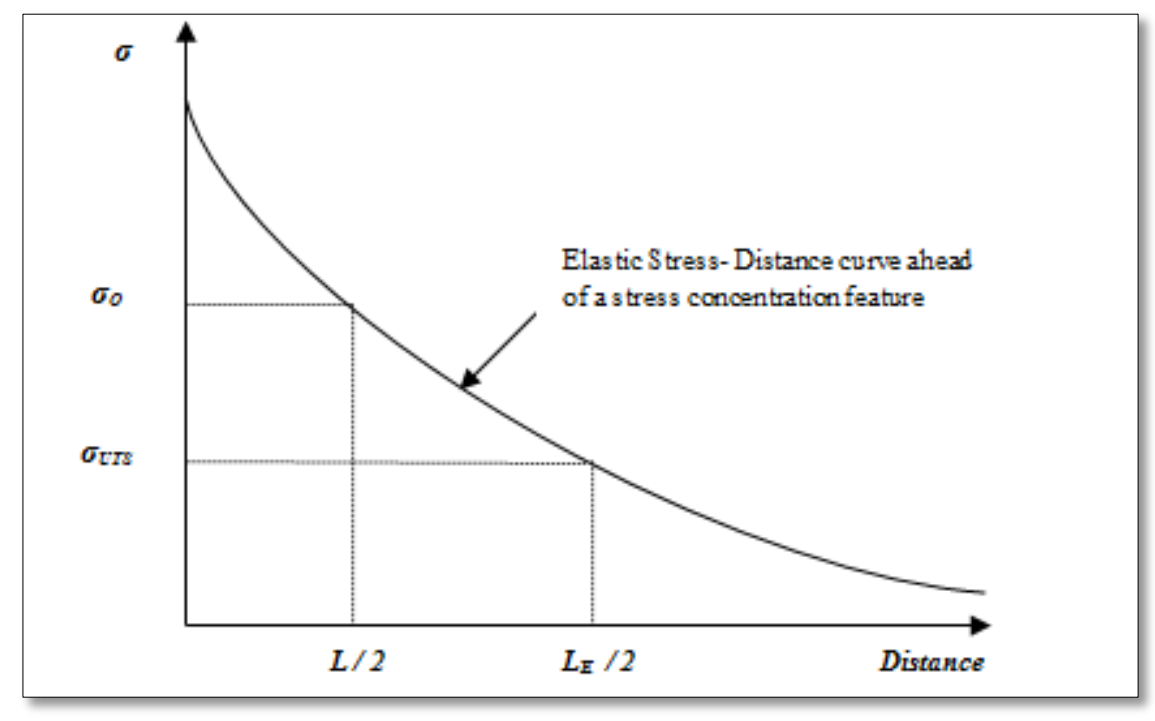

Figure 3 - Failure Stress and Critical Distance Relationship

David Taylor has further expanded his theories in 2009, and, in collaboration with Saeid Kasiri and Emma Brazel, he investigated how TCD could be applied to problems in the medical field, in particular for investigating fracture and fatigue of bone. Taylor discovered that a more complex TCD LM does not provide more accurate results, and he used a PM method and finite element analysis (FEA) to obtain the stress field information (Taylor, Kasiri, and Brazel, 2009).

The most recent studies regarding TCD were made by R. Louks et al. in 2014, where TCD has been applied as a method for static assessment of brittle/ductile notched materials. He reformulated the TCD for Mode I fracture assessment in brittle, quasi-brittle and ductile notched materials by measuring the required critical distance through standard mechanical properties. Based on the previous work, he proposed the following equation for calculating an engineering value for the critical distance: 


$$
L=\frac{1}{\pi}\left(\frac{K_{I C}}{\sigma_{u t s}}\right)
$$

According to the TCD PM, the component failure was assumed to happen when the effective stress at $\frac{L_{E}}{2}$ along the notch bisector reaches the UTS (Figure 3). Prediction error was calculated for each data set in Luoks et al. study, and the conventional method was proven to predict static failures with an accuracy of $\pm 15 \%$ error (Luoks et al., 2014). 


\section{Chapter 2 - Literature Review}

\subsection{Application of Theory of Critical Distances in Hydraulic Fracturing}

The theory of critical distances (TCD) can be inclined towards predicting the fracture of rocks and minerals because of their classification as brittle, ceramic materials, and their similarity with the previously studied specimens (Taylor, The theory of critical distances. A new perspective in fracture mechanics, 2007). As a matter of fact, these geological materials have low strengths and grained structure, and, alike concrete and other earlier studied building materials, they might experience large $L$ values. For example, there is an evidence of $\mathrm{L}$ value reaching several meters in sea ice (Dempsey, 1999). This is the largest specimen ever made, where the square sheets of floating ice ranged from 0.5 to 80 meters long.

In 1971 Lajtai introduced the first application of the theory of critical distances in hydraulic fracturing (Lajtai, 1971), when he incorporated both the maximum stress concentration and the stress gradient in his theory. In his work, Lajtai studied the problem of fracture initiation of a rock and determined that the fracture initiation depends not only on the maximum stress concentration, but also on the stress gradient along the fracture path. His paper was limited to the description of a few test sets, which involve the visual effect of stress gradient. They further lead towards the development of a theoretical model to comprehend the experiments.

Lajtai used the concept of the Griffith theory (Griffith, 1921) and his extensions and modifications in his study, which state that the fracture is initiated when the maximum tensile stress concentration at the boundary of critically oriented crack $\left(\sigma_{m}\right)$ reaches the uniaxial tensile strength of the material near the crack:

$$
\sigma_{m}=T_{o}
$$

Lajtai included stress gradient in his study by claiming that the fracture initiation occurs when the maximum tensile stress $\left(\sigma_{d}\right)$ at a certain distance $d$ along the fracture path reaches the uniaxial tensile strength. The distance $d$ at which the critical stress $\sigma_{d}$ occurs has been randomly selected. The critical distance $d$ was considered as a material constant, and its main function is to demonstrate the 'size effect'. Based on experimental work, size effect is important, and it may be a most significant parameter for small cracks. However, the issue of 'size effect' was rather complicated in this study, because the same crosssectional area of a crack could have different fracture orientations at different stress levels. The Figure 4 shows that the intensity of the stress field at a given distance $d$, in contrast to the maximum stress concentration $\sigma_{m}$, is a function of flaw size. The value of $d$ indicates the brittleness of a material. Brittle materials, which have high critical stress concentration $\sigma_{d}$, are very close to the boundary (Figure 4).

Ito and Hayashi carried on the previous discovery made by Lajtai in their work, where they applied the theory of critical distances point method (TCD PM) towards studies on hydraulic fracturing of rocks (Ito and Hayashi, 1991). They presented a new theory on the critical wellbore pressure at fracture initiation in the hydraulic fracturing tectonic stress measurements. Their study explained that the fracture initiation depends not only on the maximum stress concentration but also on the stress gradient along the path. This work introduced the dependency of the pressure on the wellbore diameter and pressure rate, and it 
extended the previous study to the fracture initiation of the porous permeable rock with pore fluid. The results showed that experimental breakdown pressures decrease with the increasing wellbore diameter.

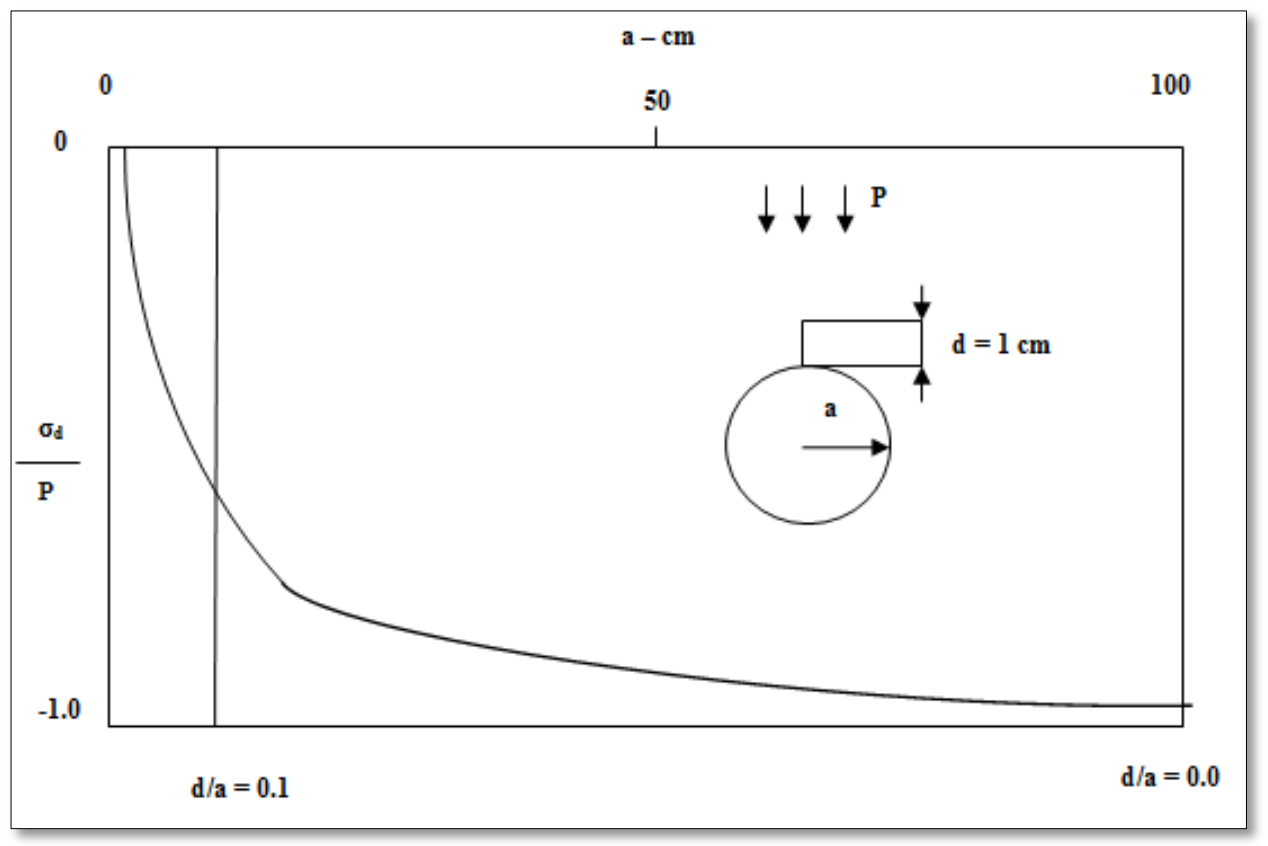

Figure 4 - Tensile stress intensity at a distance beyond the periphery of a circular void. In contrast to 'maximum stress concentration', stress intensity at a constant distance is influenced by the absolute size of the void

(Lajtai, 1971)

\subsection{Previous Work in Optimization Fracture Spacing and Sequencing in Horizontal Well Fracturing}

Optimization of multistage hydraulic fracturing has an evident impact on well production in unconventional oil and gas reservoirs. The shift towards developing unconventional resources, accordingly, has carried along some studies related to multistage hydraulic fracturing, particularly, optimization of a number of fractures and fracture spacing. However, simplified models used for multistage hydraulic fracturing design resulted in significant practical problems including merging hydraulic fracturing stages and reducing the efficiency of the stimulation. Thus, quantitative analysis of the magnitude of the induced stress and in-situ stress reorientation with dynamics of net fluid pressure is of particular significance.

Previous studies in the literature, related to induced stresses and in-situ stress reorientation, mostly focused on the effect of a single fracture (Siebrits et al., 1998). Soliman and Adams first calculated the effect of multiple fractures on stress magnitude and net pressure by using analytical solutions (Soliman and Adams, 2004). Both studies showed a growth in stress values with increased number of sequenced fractures and decreased fracture spacing. The stress field and the fracture geometries were calculated by using static numerical model based on the displacement discontinuity method. The studies were performed on three fractures of the homogeneous single-layer formation (Cheng, 2009). 
"Stress shadowing" effect has been discussed in the literature since early 90's. However, all cases were restricted for tightly-spaced wellbores and were mostly due to long-term production / injection operations. Fisher et al. (2004) first introduced "stress shadowing" as a phenomenon where induced by hydraulic fracture stress changes the local maximum/minimum horizontal stress ratios and the direction of subsequent hydraulic fracture in horizontal well fracturing. He showed stress shadow effect on transverse fracture growth, and displayed the rotation of preferred fracture plane inside stress shadow.

Nicholas Roussel and Mukul Sharma (2011) quantified the effect of reservoir's mechanical properties on the 3-D magnitude of stress reorientation caused by an open crack. Their study took into account the presence of the bounding layers with mechanical properties different from pay zone (Figure 5). They also investigated the fracture vertical growth into the bounding layers.

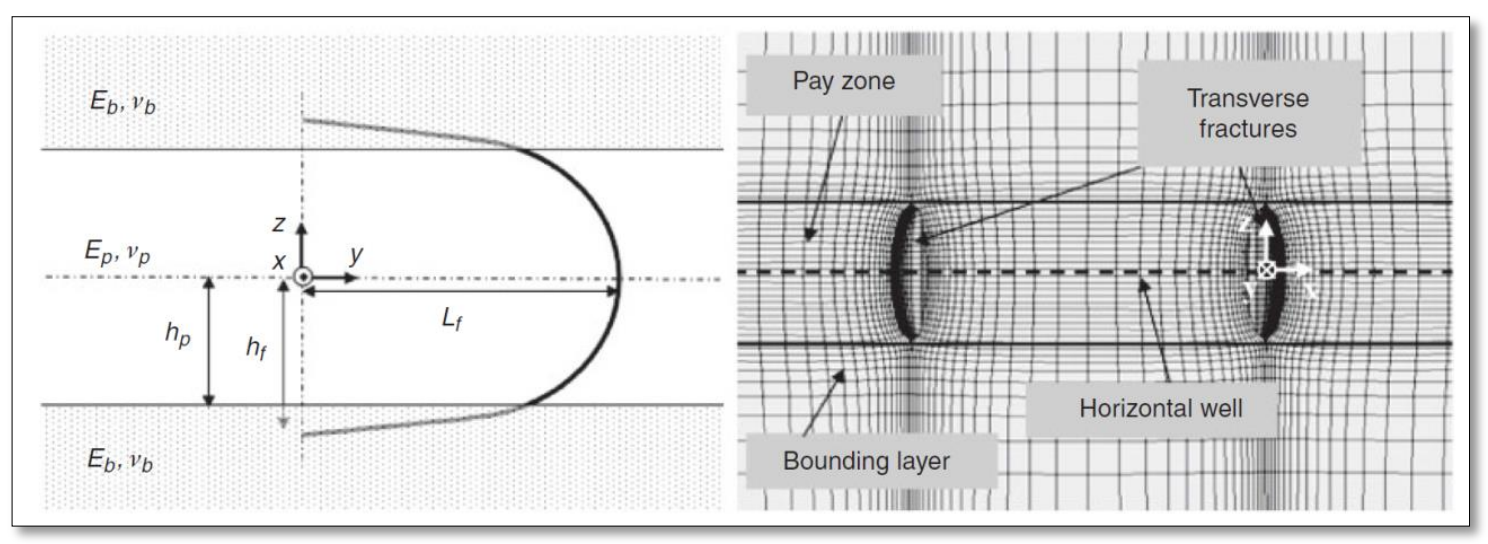

Figure 5 - 3-D model of multiple horizontal fractures in a reservoir with bounding layers

(Nicholas Roussel, Mukul Sharma, 2011)

The magnitude of stresses generated with fracture growth is a function of fracture dimensions $\left(w_{o}, h_{f}, L_{f}\right)$, Poisson's ratio in the pay zone $\left(v_{p}\right)$, the ratio of Young's moduli of the bounding layers and the reservoir $\left(E_{b} / E_{p}\right)$, and fracture dispersion into the bounding layers $\left(h_{f} / h_{p}\right)$. To avoid fracture merging, fracture spacing should be larger than the distance between fracture and the end of $5^{\circ}$ stress-reorientation region that can be calculated in the model (Figure 6). In other words, optimum fracture spacing is defined as a distance between fracture and the end of the stress-reversal region, known as 'isotropic point'. In this work, stress reversal region is described as an area where the direction of maximum horizontal stress is parallel to the horizontal well. Fracture initiation within stress reorientation region may either lead to fracture longitudinal growth or to the well screen out, caused by the accelerated change of fracture orientation in this area.

However, an introduced above study still has the following limitations:

- The pay zone of the model is homogeneous, isotropic and elastic

- After the first fracture has propagated, its geometry is fixed, and no displacement is allowed

- Net pressure is assumed to be constant along the fracture. Therefore, the model used for this research is not fully coupled

- Natural fracture network has not been incorporated in this study 


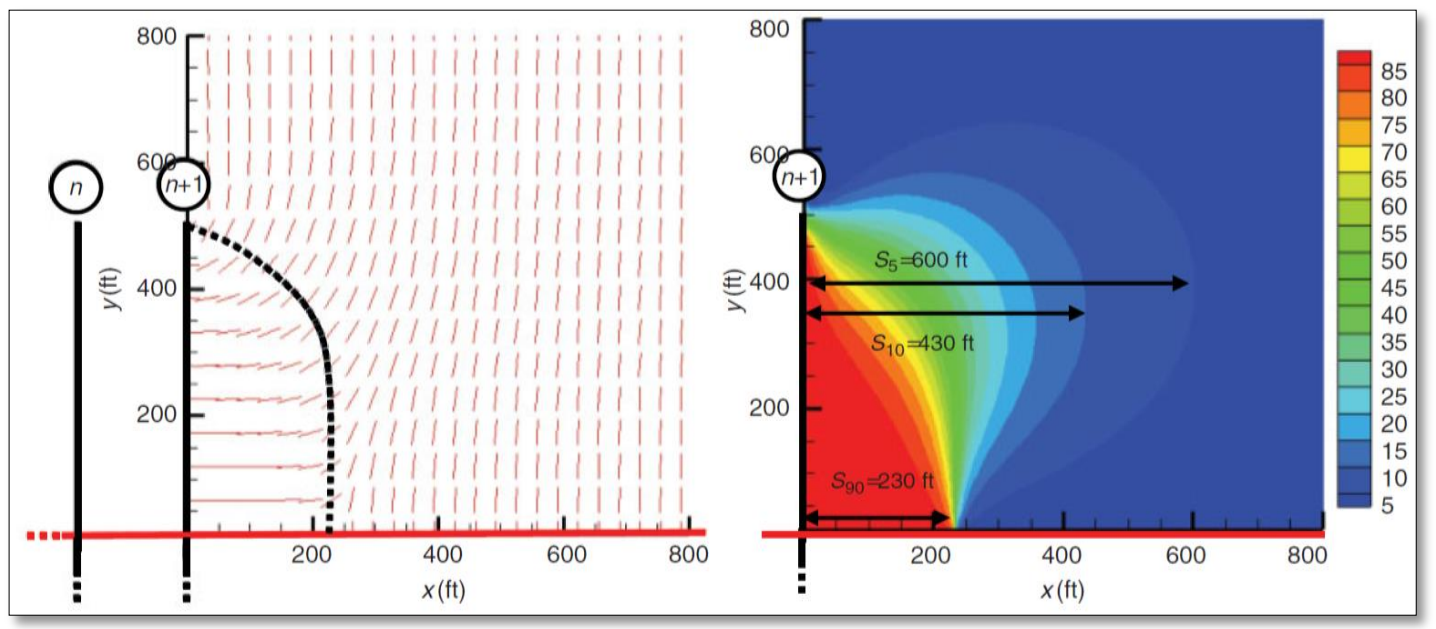

Figure 6 - Direction of maximum horizontal stress and angle of stress orientation from multiple transverse fractures (Nicolas Roussel, Mukul Sharma, 2011)

Roussel and Sharma have made a comparison of stress reorientation due to poroelastic effect and mechanical effects in their study, and they came to the conclusion that poroelastic effect can be neglected in fracturing of horizontal wells because the production and injection of fluids are minimal (Roussel and Sharma, 2011). However, in cases when large volumes of fluid have been produced from the well, poroelastic effect dominates mechanical effects. Figure 7 illustrates how stress distribution affected by fracture opening differs from the one caused by poroelastic stresses. In the wells with a significant volume of produced fluid, the orientation of maximum horizontal stress is rotated $90^{\circ}$ from its in-situ stress direction. Also, stress orientation is not limited to the stress-reversal region. However, outside the stress reversal region, the direction of maximum horizontal stress is in the orthoradial direction towards the fracture. In contrast, when mechanical effects are dominant, stress reorientation is limited with a stress-reversal region, and the direction of maximum horizontal stress outside of this region is pointing towards the fracture. In addition, the angle of stress orientation in the vicinity of the fracture is relatively smooth.

Jonathan Morrill and Jennifer Miskimins performed sensitivities on different mechanical and reservoir properties in 2011, and they discovered which properties are the most important in the determination of the minimum fracture spacing without stress interference by studying how and why the stress field changed. They found the ratio of minimum to maximum horizontal stress to be a key parameter in defining the fracture spacing. The stress reversal region did not appear in this sensitivity until their model had a stress ratio of 0.94. However, in the area surrounding fracture tip, even low-stress ratios led to significant change in the direction of maximum horizontal stress (Figure 8). The other important parameters were Poisson's ratio, Biot's parameter, and net fracture pressure. The simulator, coupling porous media flow with geomechanical stress analysis, was applied in this study, and it was setup to generate dynamically far-field stresses in the reservoir during the simulation run. The major component of the sensitivity analysis is an assumption that the stress shadow effect is limited to the distance from the first hydraulic fracture to the part of the reservoir where the direction of maximum horizontal stress is altered by five degrees $\left(5^{\circ}\right)$ or less along the length of the fracture. This assumption was made arbitrary. However, having a criterion of the altered direction of maximum horizontal stress to be less than five 
degrees would lead to the similar results. As a matter of fact, the five-degree assumption was also made by Nicholas Roussel and Mukul Sharma in the previously mentioned study.

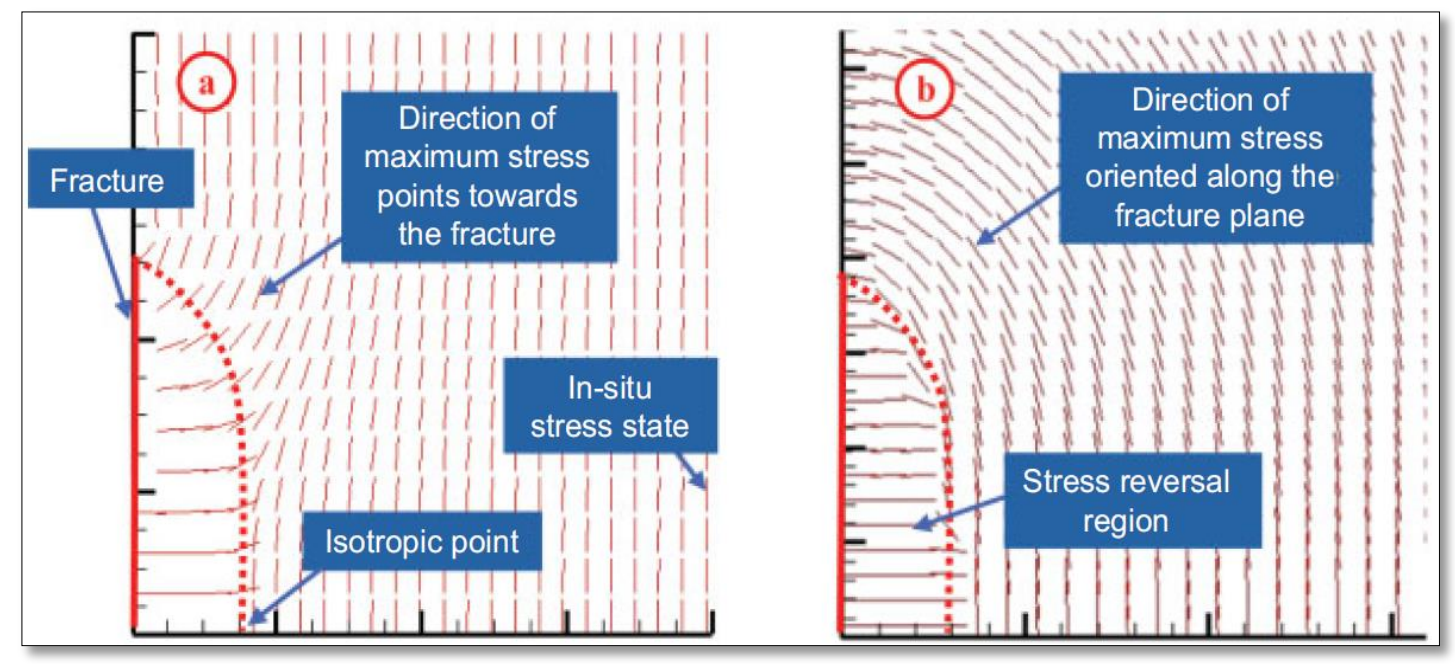

Figure 7 - Maximum horizontal stress reorientation caused by (a) mechanical effects and (b) poroelastic effects (Nicholas Roussel, Mukul Sharma, 2011)

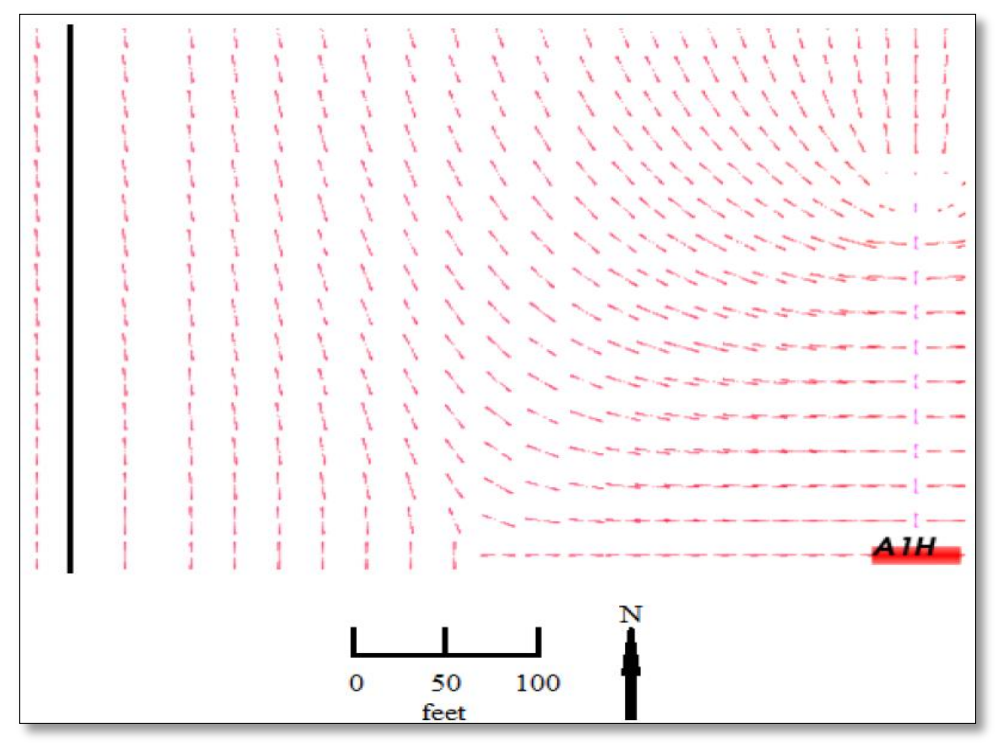

Figure 8 - Direction of maximum horizontal stress for the case of minimum/maximum stress ratio of 0.99 . The recommended fracture spacing is $\mathbf{4 8 3}$ feet, where the reorientation of maximum horizontal stress is down to five degrees (J. Morrill, J. Miskimins, 2012)

The sensitivity analysis showed the following observations:

- The minimum fracture spacing is increased with the increase of the pressure in the fracture (Figure 9).

- The higher value of Young's modulus causes the higher stress field. However, small changes in Young's modulus do not cause any effect on fracture spacing. 
- The higher value of Poisson's ratio, the larger fracture spacing has to be assigned to avoid fracture interaction. Even minor change in Poisson's ratio value may lead to the significant modification of the stress field around the hydraulic fracture.

- The change in the ratio of minimum to maximum horizontal stress shows an exponential change in fracture spacing. In the case of the high value of their ratio (0.99), the stress reversal region becomes almost circular (Figure 8).

- The lower value of Biot's number translates into a smaller distance from the fracture, needed to fit the five-degree criteria.

The described above research has been conducted based on a single fracture in the homogeneous model. (Morrill and Miskimins, 2012).

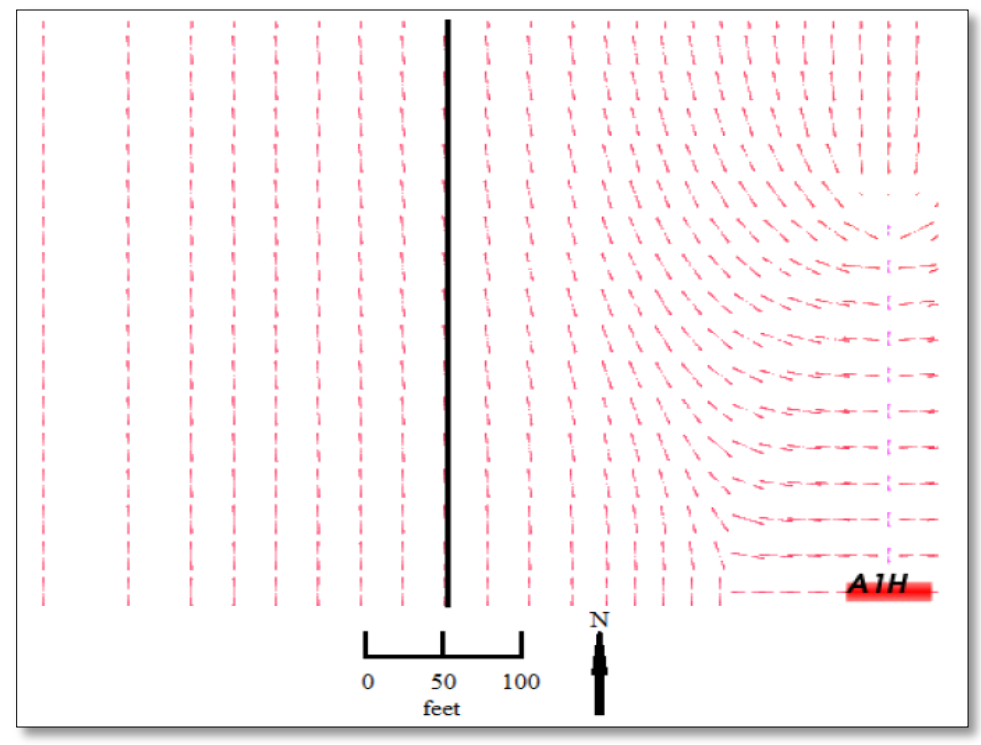

Figure 9 - Direction of maximum horizontal stress for the net pressure of 3,500 psi. The direction of maximum horizontal stress has reoriented 90 degrees near the fracture. The recommended fracture spacing is $\mathbf{2 6 7}$ feet, where the reorientation of maximum horizontal stress is down to five degrees

(J. Morrill, J. Miskimins, 2012)

The previous study, however, mentioned that because stress ratios in shale can be close to one (Roussel and Sharma, 2011), the significance of this parameter in fracture design can be neglected, and the sequenced fractures might be placed in zones of reoriented maximum horizontal stress. Therefore, it is important to study the stress ratios and their impact on fracture spacing.

\subsection{Fundamentals of Geomechanical Modeling}

Despite all developments that occurred in numerical simulation of hydraulic fracturing for the past 15 years, the same basic theory of fracture propagation has been applied since 1920's and used in the original 2-D fracture models - known as Linear Elastic Fracture Mechanics, or LEFM. The small modifications of LEFM theory were made mainly to allow for the effects of non-linearity at the crack tip. The major assumption of the theory still stayed true and stated that failure is by brittle fracture, with no significant deformation of the rock, including at the fracture tip. This assumption can be valid for the majority of 
formations, and is applicable in nowadays industry. However, very soft formations would demonstrate non-linear strain with significant plastic deformation, and LEFM theory would not be reliable. Fortunately, there is another way to model fracture propagation called Crack Tip Plasticity (CTP) method. CTP assumes a fracture tip of finite radius, with a zone of plastically deformed material around it. Fractures with substantial plastic deformation are harder to propagate since the plastic zone acts to absorb extra energy from the fracturing fluid. In other words, for a given ductile material, the fractures will be smaller and less conductive comparing to those predicted by LEFM. Therefore, CTP helps to define how much smaller the fractures are. Besides, the major differences in results achieved from LEFM and CTP models, indicate that LEFM-based fracture simulators are not capable of providing accurate models for very soft formations. The concepts of CTP have been applied in fracture mechanics so far, but they seem to be never practically used in hydraulic fracturing due to its complexity.

\subsubsection{Fracture Mechanics and Theory of Elasticity}

Fracture mechanics studies state properties of solid materials and the mechanical behavior of crack-free or cracked solids. Force, load, strain, and displacement are the main variables, and they are essential parameters for characterization of the mechanical behavior of the solids. There are two areas of fracture mechanics: Linear Elastic Fracture Mechanics (LEFM), based on linear elasticity theory, and Plastic Fracture Mechanics (PFM), covering plastic behavior of ductile solids.

An important engineering parameter in theory of elasticity is elastic stress:

$$
\sigma=\frac{P}{A}
$$

where $P$ - mechanical force, analogous to load, and $A$ - original cross-sectional area.

The strain is caused by stress and it is defined as a change of displacement in the $\mathrm{x}, \mathrm{y}$ and $\mathrm{y}$-directions:

$$
\begin{gathered}
\epsilon=d \mu / d x, \\
\epsilon=d \mu_{x} / d y \\
\epsilon_{z z}=d \mu_{z} / d z
\end{gathered}
$$

where $d \mu$ - change of displacement.

One of the main purposes of the theory of elasticity is to determine a point of stress when the material will start fracture, known as Tensile Strength (Fracture Stress). An observation of 'crack propagation', when the fracture initiates, indicate a brittle material. However, a point of stress when a material deforms permanently is defined as Yield Strength (Yield Stress).

Hook's Law of elasticity is a basis of the theory of elasticity. It states that the load is in direct proportion with the displacement of material as long as it does not exceed elastic limit, and can be applied to linear elastic materials: 


$$
F=k X
$$

For an elastic deformation process, Hook's law defines the tensile and shear modulus as:

$$
\begin{gathered}
E=\frac{\text { Tensile Stress }}{\text { Strain }}=\frac{\sigma}{x / a_{0}} \\
G=\frac{\text { Shear Stress }}{\text { Strain }}=\frac{\tau}{x / a_{0}}
\end{gathered}
$$

where $a_{0}=\lambda$ - equilibrium atomic distance.

Young's modulus represents the stiffness of a material, and it displays how much a material will stretch as a result of a caused stress:

$$
E=\frac{\sigma}{\epsilon}
$$

where $E$ - elastic modulus of elasticity

$$
\begin{aligned}
& \sigma-\text { elastic stress } \\
& \epsilon-\text { strain. }
\end{aligned}
$$

The stress intensity factor $K_{l}$ leads to crack growth, and its critical value is fracture toughness (material property), which is the resistance force to crack propagation:

$$
K_{I}=\sigma \sqrt{\pi a}
$$

The critical value of $K_{I}$ (fracture toughness) can be calculated at a fracture stress when the crack length reaches a critical or maximum value before the rapid crack growth occurs. Fracture toughness is also known as the elastic strain energy density $W\left(\right.$ Joules $\left./ \mathrm{m}^{3}\right)$ and can be defined as:

$$
W=\int_{0}^{\epsilon} \sigma \mathrm{d} \epsilon
$$

An ideal tough material demonstrates high strength and ductility. The yield strength and the fracture toughness of crack-free materials can be compared with the following inequalities:

$$
\begin{gathered}
\sigma_{y S}{ }^{\text {ductile }}<\sigma_{y s}^{\text {tough }}<\sigma_{y s}{ }^{\text {brittle }} \\
W_{\text {brittle }}<W_{\text {tough }}<W_{\text {ductile }}
\end{gathered}
$$

However, the above expressions can be applied only for solid materials. They indicate that increasing strength and decreasing strain to failure cause the yield strength decrease and the total strain energy density increase. Practical engineering applications do not require a high ductility most of the time. Thus the above inequalities should be modified accordingly:

$$
\sigma_{y s}{ }^{\text {ductile }}<\sigma_{y s}^{\text {tough }} \approx \sigma_{y s}^{\text {brittle }}
$$




$$
W_{\text {brittle }}<W_{\text {tough }} \approx W_{\text {ductile }}
$$

Equilibrium Equations in Cartesian coordinates are the sum of forces at equilibrium. They can be utilized for deriving analytical solutions for the unknown stresses $\sigma_{x x} \sigma_{y y}$ and $\tau_{x y}$ :

$$
\begin{aligned}
& \frac{\partial \sigma_{x x}}{\partial x}+\frac{\partial \tau_{x y}}{\partial y}+F_{X}=0 \\
& \frac{\partial \sigma_{y y}}{\partial y}+\frac{\partial \tau_{x y}}{\partial x}+F y=0
\end{aligned}
$$

For the solution of elasticity problem and determination of the stresses we use Airy's stress function $\phi$, and base further calculations on the fact that $\phi$ must satisfy a biharmonic equation in the order of $\Delta^{4} \phi=0$, the equilibrium and compatibility equations, and boundary conditions. The Airy's stress function can be used for analytical calculations of unknown stresses $\sigma_{x x}, \sigma_{y y \text { and }} \tau_{x y}$ in two-dimensional elasticity problem.

$$
\begin{gathered}
\sigma_{x x}=\frac{\partial^{2} \phi}{\partial y^{2}}+\Omega \\
\sigma_{y y}=\frac{\partial^{2} \phi}{\partial x^{2}}+\Omega \\
\tau_{x y}=-\frac{\partial^{2} \phi}{\partial x \partial y}
\end{gathered}
$$

Where $\Omega=\Omega(x, y)$ is the body force field.

The Airy's biharmonic equation is following:

$$
\Delta^{4} \phi=\frac{\partial^{4} \phi}{\partial x^{4}}+2 \frac{\partial^{4} \phi}{\partial x^{2} \partial y^{2}}+\frac{\partial^{4} \phi}{\partial y^{4}}=0
$$

\subsubsection{Linear Elastic Fracture Mechanics (LEFM)}

\subsubsection{The Griffith Crack}

Griffith offered the first analytical approach to fracture mechanics in 1920's (Griffith, 1921, 1924), and the concept of his approach is shown on Figure 10.

The Griffith crack can be expressed with an equation below:

$$
\frac{\delta U}{\delta a}=\frac{2 \pi \sigma^{2} a}{E}
$$

where $U$ - elastic energy or energy, used to produce elastic stress on the material

$a$ - characteristic fracture length

$\sigma-$ far field stress (stress away influenced by the fracture)

$E-$ Young's modulus 
In other words, the above equation, which describes the concept of Griffith crack, shows the amount of additional energy $(\delta U)$, needed to cause the fracture growth from length $a$ to length $a+\delta a$.

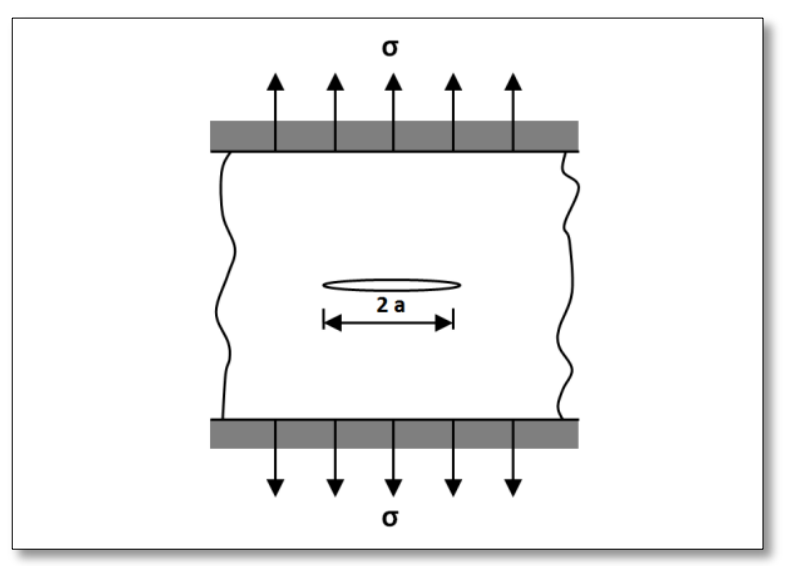

Figure 10 - The Griffith Crack

By replacing $\delta U / \delta a$ with $2 G$, we are getting another expression of the Griffith crack concept, where $G$ is described as the "elastic energy release rate" or "crack driving force":

$$
G=\frac{\pi \sigma^{2} a}{E}
$$

However, Griffith does not account for a fracture tip in his studies. He assumes that energy is either used to deform elastically the material or to rupture the material. Therefore, his model is applicable only for materials, experiencing elastic deformation followed by brittle fracture with no deformation at the fracture tip.

Moving towards a model accounting for plastic deformation, the total elastic-plastic strain energy has been introduced in the following equation, and it has a definition of the strain energy release rate $G_{I .}$ It can be also described as energy per unit crack surface area existing for an infinitesimal crack extension.

$$
G_{I}=2\left(\gamma_{s}+\gamma_{p}\right)
$$

where $\gamma_{s}$ - specific surface energy

$\gamma_{p}$ - plastic deformation energy or plastic strain work

According to the above expression, Irwin and Orowan upscaled Griffith's elastic surface energy equation by including a plastic deformation energy, also known as plastic strain work, $\gamma_{p}$, in the fracture process for accounting plastic deformation. In case of high plasticity at the crack tip the crack blunts and its radius of curvature increases. The temperature and microstructure also influence this plastic deformation process. Although crack tip plasticity has been applied in fracture mechanics, it has never been used in hydraulic fracturing. 


\subsubsection{Griffith Failure Criterion}

Assuming that material has uniform properties and $\delta U / \delta a$ stays constant, Griffith offers a critical value of stress $\sigma_{c}$, at which material experiences catastrophic failure:

$$
\sigma_{c}=\sqrt{\frac{E G_{I c}}{\pi a}}
$$

where $G_{I C}-$ critical energy release rate for failure mode I or "opening mode", which is defined experimentally and it is a material property. It is a function of temperature and the geometry of test specimen. In case when stress is given, the above equation allows determining a critical fracture length, above which the material will fail.

\subsubsection{Stress Intensity Factor}

The stresses in the principal directions at any point away from the fracture tip can be expressed through the following equations (Broek, 1986; Hudson and Harrison, 1997, Economides and Nolte, 1987):

$$
\begin{aligned}
\sigma_{x x} & =\frac{K}{\sqrt{2 \pi r}} \cos \frac{\theta}{2}\left(1-\sin \frac{\theta}{2} \sin \frac{3 \theta}{2}\right) \\
\sigma_{y y} & =\frac{K}{\sqrt{2 \pi r}} \cos \frac{\theta}{2}\left(1+\sin \frac{\theta}{2} \sin \frac{3 \theta}{2}\right)
\end{aligned}
$$

where $K-$ stress intensity factor. Figure 11 illustrates the coordinate system for stress intensity factor, $K$. Further modifications can be made, by considering the plain strain case (Figure 12):

$$
\sigma_{y y}=\frac{K}{\sqrt{2 \pi r}}
$$

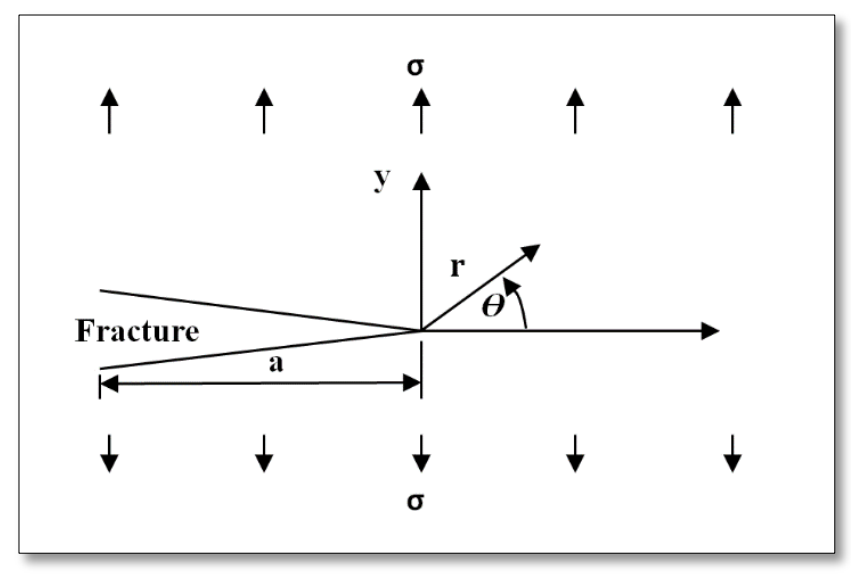

Figure 11 - Coordinate System for Stress Intensity Factor, K 


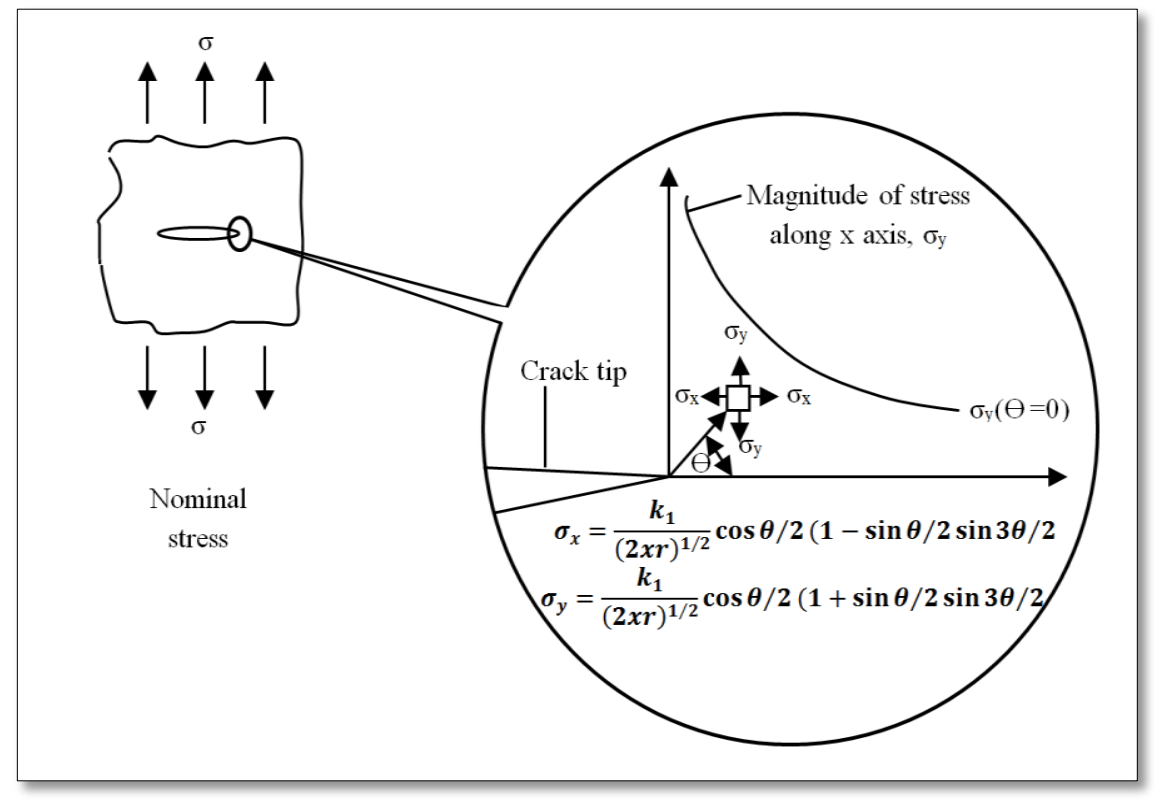

Figure 12 - Distribution of stresses near the tip of a through-thickness crack in a plate

The plain strain case with a negligible z-axis was assumed as follows:

$$
\left\{\begin{array}{c}
\varepsilon_{z z}=0 \\
\theta=0 \\
a \gg r
\end{array}\right.
$$

In this case, thickness is large enough to neglect strain on the z-axis, and, since $\cong 0$, then $\sigma_{y y} \cong \infty$, such a modeling will fail close to the fracture tip, and will not provide accurate results. In this approach, $K$ is the only factor (material property) that influences the magnitude of stress at a given distance from the fracture tip. $K$ relationship with length and stress is given below, and it can be solved either analytically or numerically:

$$
K=\mathrm{Y} \sigma \sqrt{a}
$$

where $\mathrm{Y}$ - elastic geometry factor, which value is known for most common applications.

A critical stress intensity factor $K_{I c}$, known as fracture toughness, helps to describe critical stress $\sigma_{c}$ at material failure. This following equation is known as the fundamental equation of LEFM:

$$
\sigma_{c}=\frac{K_{I c}}{\beta \sqrt{\pi a}}
$$

where $\beta$ - geometrical factor, equal to $\mathrm{Y} / \sqrt{\pi}$

Stress intensity factor is involved in the calculation of the amount of energy that material can absorb before it starts fracturing:

$$
G_{I C}=\left(1-v^{2}\right) \frac{K_{I c}^{2}}{E}
$$


where $v-$ Poisson's ratio.

The stress intensity factor solutions are given as equations or look-up tables, which can be selected along with the background information and relevant references. There is also an option of deriving stress intensity factor solutions from alternative approaches, such as finite element analysis or weight function method.

The weight function procedure, developed by Buckner, offers a calculation of the stress intensity factor for each given stress distribution and each crack length. The stress intensity factors can be determined by multiplying the weight function by the stress distribution and integrating it along the crack length:

$$
\begin{aligned}
K_{I} & =\int_{0}^{a} \sigma(x) h_{I}(x, a) d x \\
K_{I I} & =\int_{0}^{a} \tau(x) h_{I I}(x, a) d x
\end{aligned}
$$

The weight function $h(x, a)$ depends only on the geometry of the component, and it is not affected by the stress distribution.

\subsubsection{Modeling Based on LEFM}

Nowadays LEFM is extensively used for modeling of fracture propagation. Initially, LEFM concepts were applied in 2-D models, such as PKN (Perkins, 1961) or KGD (Zheltov and Khristianovitc, 1955). Today most of fracture propagation simulators in oil and gas industry are based on one of two approaches: Cleary et al. or Meyer et al.

The user of any hydraulic fracturing software based on the Cleary et al. approach (i.e. FracPro and FracproPT) can observe that changes to input fracture toughness have a very little or zero influence on fracture geometry. Therefore, the Cleary et al. approach is not based on the concept of fracture toughness. The theory states that confining stress has much more effect than fracture toughness (Cleary, 1991).

Moreover, fracture toughness can be neglected if the following condition is satisfied:

$$
\sigma \sqrt{\pi R} \gg K_{I C}
$$

where $R$ - radius of the fracture, equal to the LEFM characteristic fracture length.

The above equation shows that "fracture toughness is more significant in shallow formations, such as during skin bypass fracturing” (Rae, Martin and Sinanan, 1999).

The injection fluid doesn't penetrate to the end of the fracture (Pater et al., 1994; Greetsma, 1994). It indicates that there is a noticeable change in net pressure at a distance $w$ from the fracture tip (Figure 13). 


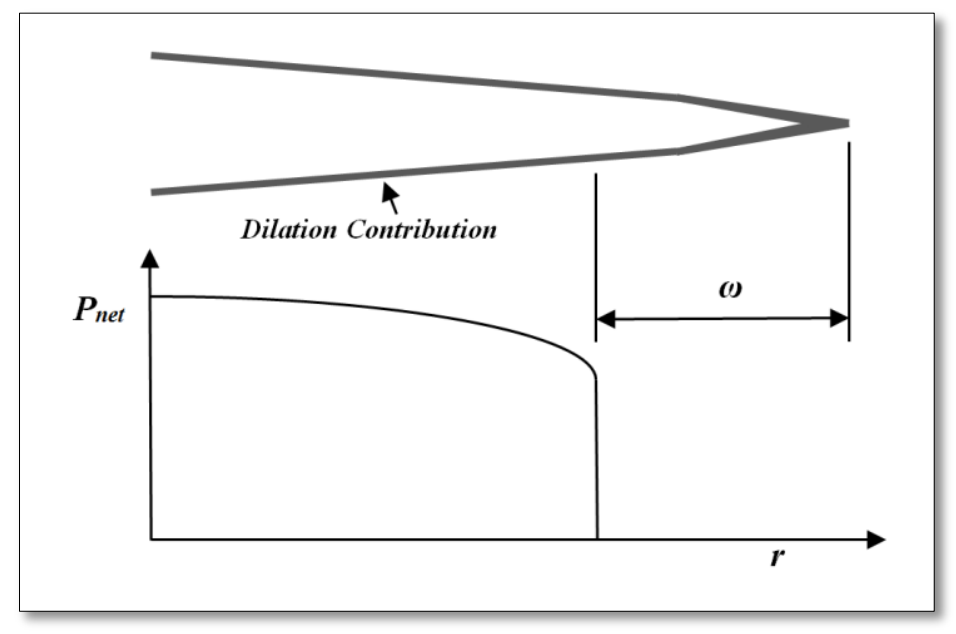

Figure 13 - The Clearly et al approach

If Equation 44 is satisfied, $w$ can be determined as:

$$
w \approx \frac{R}{2}\left(\frac{P_{n e t}}{P_{n e t}+\sigma_{c}}\right)^{2}
$$

Since the injection fluid does not penetrate into fracture tip, energy is lost as the tip of the fracture deforms. This non-linear or dilatant deformation reduces the size of the fracture for $P_{\text {net }}$ due to energy loss.

Meyer et al. approach is applied in MFrac software, and its methodology is very close to the original LEFM approach. The main concept of the Meyer et al approach is also a basic LEFM criterion which states that, stress intensity factor $K$ has to overcome fracture toughness $K_{I C}$ in order for a fracture to start propagating. At material failure, the critical stress $\sigma_{c}$ is described through characteristic length $H_{\xi}$ and a geometry factor $\gamma$ (mentioned as $\beta$ in Equation 40) in the conventional LEFM equation:

$$
\sigma_{c}=\frac{K_{I c}}{\sqrt{\gamma H_{\xi}}}
$$

The geometry factor $\gamma$ depends on the type of fracture model: PKN, KGD, Ellipsoidal or 3-D model. The characteristic length for a 3-D model is found from a set of partial differential equations. They relate mass conservation, mass continuity, momentum conservation, and vertical and lateral propagation rates (Meyer, 1989).

MFrac does not account for a "tip over-pressure"; it is one of the software that handles this issue by applying "over-pressure factor", which can be defined empirically, or by using large values of fracture toughness.

Comparison studies between Clearly et al. and Meyer et al. methods have been performed (Warpinski et al., 1993; Cleary, 1994) and illustrated a significant difference in fracture geometries while having an identical set of input data. Cleary et al. model predicts fractures with smaller length and greater height 
than the Meyers et al model. FracPro and FracproPT (based on the Cleary et al approach) and MFrac (based on the Meyer et al approach) are the three main simulation models used in the industry today, and it is believed, that Meyer model works best in "soft" formations, while Cleary model is best for "hard" formations. However, it is just an assumption due to the origins of the models (Martin, 2000).

It has been shown that making an accurate modeling of fracture height with mentioned above approaches can be unachievable due to the missing fracture tip effect (Cramer, 1995, 1996). There were several attempts at introducing other approaches of modeling fracture propagation by getting away from LEFM. So far, none of these approaches have been accepted by the industry for a myriad of reasons. Some of the models are listed below:

1. Propagation by Tensile Failure (Barree, 1983)

2. Continuum Damage Mechanics (Valko, Economides, 1993)

3. Apparent Fracture Toughness (Shlyapobersky, 1988; Shlyapobersky, Chudnovsky, 1994)

4. Crack Layer Concept (Chudnovsky, 1984; Chudnovsky et al., 1996)

5. Process Zone Theory (Chudnovsky, et al., 1996)

\subsubsection{Crack Tip Plasticity (CTP)}

The vast majority of hydraulic fracturing simulators are based on LEFM. The tip over-pressure still cannot be accurately accounted for due to several mechanisms working at the same time. Various formations have different amounts of these mechanisms. However, none of the models account for a significant plastic zone at the tip of the fracture (Martin, 2000).

Even though the plastic deformation has been neglected in hydraulic fracturing so far, it may be not significant for some formations. Nevertheless, the mechanical response of some formations above and under the reservoir formation can significantly affect the fracture geometry. Amongst them are softer carbonate formations (chalks), high-temperature formations, "plastic" formations (such as halites or other evaporites), highly over-stressed formations, and coal seams (Martin, 2000).

Unlike LEFM, where stresses are rising asymptotically to infinity at a fracture tip singularity, the CTP method states that the stresses rise until the yield point of the rock is reached $\left(\sigma_{y}\right)$. The rock starts deforming plastically at this point, and the plastic flow absorbs energy, which prevents the stresses from rising to infinity (Figure 14).

The plastic zone reduces the stress of the solid formation, and the rock deforms away from the region of high stress at the fracture tip. It causes the formation of blunt fracture tip with plastic region around the fracture tip of radius $r_{p}$ (Figure 15).

The crack tip opening diameter $d$, can be found by using the Dugdale approach (Dugdale, 1990), and it can be described with a following equation:

$$
d=\frac{K_{I}^{2}\left(1-v^{2}\right)}{E \sigma_{y}}
$$




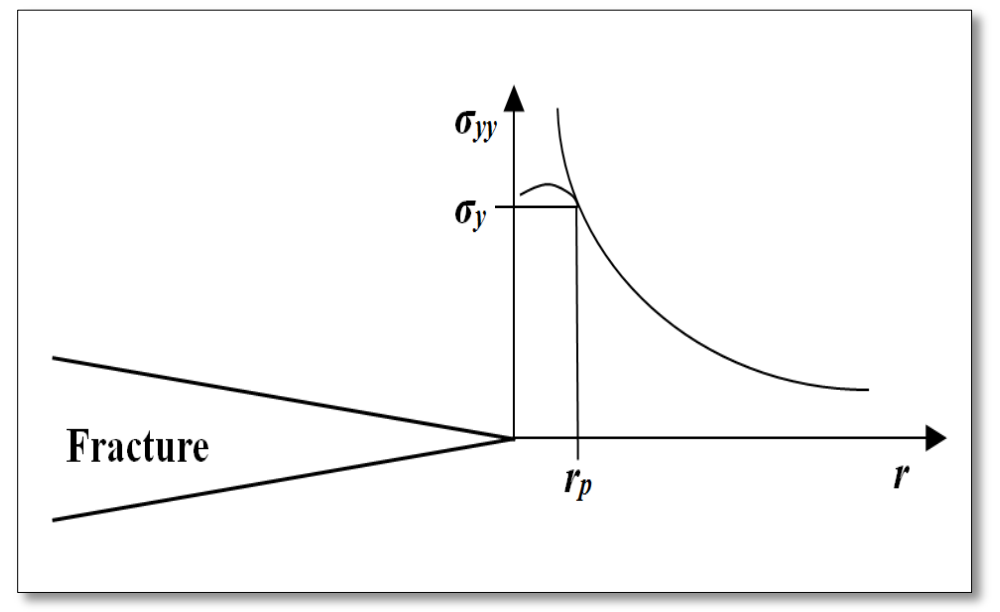

Figure 14 - The Effect of Plastic Yielding on the Stress at a Distance $r$ from the fracture tip

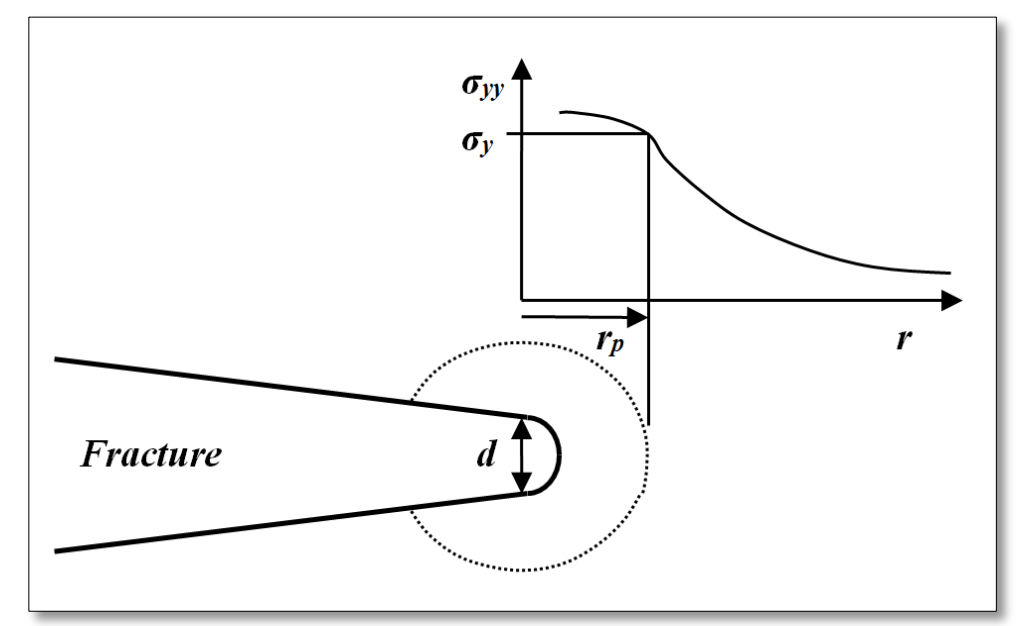

Figure 15 - Crack Tip Opening Diameter, $d$ and plastic radius, $\mathbf{r}_{p}$

The crack tip opening diameter is a significant material property in elastic-plastic fracture mechanics, which helps us define fracture toughness at material failure with the above equation (when $K_{I}$ becomes $\left.K_{I c}\right)$.

In order to compare experimental and theoretical results for plain stress and plain strain conditions, it is required to know the shape and size of the plastic zone. The plastic zone size can be calculated with the following equation:

$$
r=\frac{a}{2}\left(\frac{\sigma}{\sigma_{y s}}\right)^{2}
$$

where $\sigma$-applied Stress (MPa)

$\sigma_{y s}-$ yield Strength (MPa)

$a$ - crack Length (m) 
The plastic zone size reaches its maximum magnitude when $\sigma_{y}=\sigma_{u}$ (internal tensile stress is equal to ultimate tensile stress). Consequently the effective crack size $a_{e}=a+r$ turns into an actual size, which extends through the plastic zone as a result of coalescence and voids.

Irwin has further extended this study, and he offered to add artificially the distance $r_{l}$ to the crack, this effect is known as Irwin's plastic zone correction. It was implied to include the effect of the plastic zone. Dugdale has been working in the same area as Irwin did, and he has shown a strip yield model for the plastic zone under plane stress conditions, illustrated in Figure 16. The following comparison between Irwin's and Dugdale's approximation schemes allows us to summarize further the differences between them:

$$
r[\text { Irwin }]=\frac{8}{\pi^{2}} r[\text { Dugdale }]=0.81 r[\text { Dugdale }]
$$

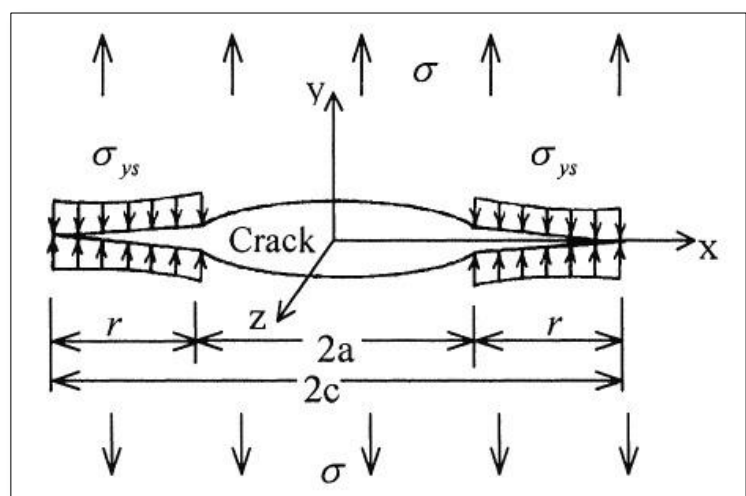

a)

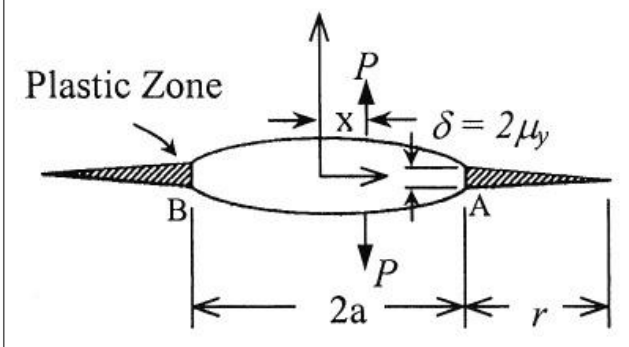

b)

Figure 16 - Dugdale Plastic Zone Strip Model for non-strain hardening solids under plane

Both methods have considerable differences in normalized stress intensity factor at large stress ratios, and should be applied carefully.

In nonlinear fracture mechanics, the crack tip opening displacement is described through models, such as the crack tip opening displacement and J-integral (J-model). The crack tip opening displacement $\left(\delta_{\mathrm{t}}=C T O D\right)$ is a measure of fracture toughness of solid materials going through a ductile-to-brittle transition and elastic-plastic or plastic behavior in large structures. 
CTOD is defined as twice the crack tip displacement in the y-direction.

$$
\begin{gathered}
\delta t=\frac{4 a \sigma^{2}}{E \sigma_{y s}}=\frac{4 K_{I}^{2}}{\pi \lambda E \sigma_{y s}} \text { (Irwin) } \\
\delta t=\frac{2 \pi a \sigma^{2}}{E \sigma_{y s}}=\frac{2 K_{I}^{2}}{E \sigma_{y s}} \text { (Dugdale) }
\end{gathered}
$$

The Von Mises criterion and Tresca Yielding Criterion can be used for deriving expressions for the plastic zone size, which consequently describes the plastic zone shape.

The Von Mises yielding criterion comes from the Maximum Distortion Energy Theory, which defines the state of stress as the key stress direction:

$$
\frac{K_{I}^{2}}{2 \pi r}\left[\frac{3}{2} \sin ^{2} \theta+h(1+\cos \theta)\right]=2 \sigma_{y s}^{2}
$$

Tresca yielding criterion is deducted from the Maximum Shear Stress Theory which states that the maximum shear stress reaches half value of the yield stress in a uniaxial tension test.

$$
\tau_{\max }=\frac{1}{2} \sigma_{y s}
$$

Both Von Mises and Tresca plastic zone shapes are different though the plain strain shape is smaller than the plane stress shape in both cases (Perez, 2004).

\subsection{D, Pseudo-3D, Planar 3D and Fully 3D Hydraulic Fracturing Simulators}

Numerical simulations are used for evaluation and prediction of the location, direction, and extent of hydraulic fractures. Depending on the degree of the wellbore and fracture geometry complexity, the necessity of results accuracy and available simulators, simulations can range from two to fully threedimensional. Most of 2D, pseudo-3D, and planar 3D hydraulic fracturing simulators perform well when the geometry of the fracture is easy to define, and it is limited to a single plane modeling. However, when a more accurate fully 3D modeling is necessary to be performed, the majority of commercial software is not capable of providing the desired results. The common mistake in the prediction of fracture propagation comes from a miscalculation of fracture parameters from deviated wellbores with arbitrary crack shapes. Most simulators simply ignore the near-wellbore effects of deviated wells and assume a single plane crack growing beyond the deviated wellbore area. The fully $3 \mathrm{D}$ simulation and accurate modeling of near-wellbore geometry are still missing and required for hydraulic fracturing of inclined wells, where cracks change their direction as they grow.

\subsubsection{D Models}

At the early stage of hydraulic fracturing modeling, two kinds of 2D models have been offered, that are still employed in nowadays simulators: Geertsma-de Klerk (KGD) and Perkins-Kern-Nordgren (PKN) models (Khristianovich and Zheltov, 1955). They both could predict the geometry of hydraulic fracture based on the formation and fluid properties, in situ stresses and pumping parameters. Both models have a 
constant height while the difference between them is that the KGD model also has a constant width through the height while the PKN model has an elliptical vertical cross-section (Carter et al., 2000).

Today, there are still only two choices available to select from, KGD $(\mathrm{L} / \mathrm{H}<1)$ and PKN $(\mathrm{L} / \mathrm{H}>1)$ 2-D models. Less often, the software has both options available, and it chooses the most applicable one for a particular simulation case. MFrac includes both PKN and KGD 2-D models, and it also offers a Penny shape 3-D model (Sneddon Model), which assumes a total fracture length to be equal to fracture height. Figure 17 shows a fracture geometry of all mentioned above models. Our fully coupled simulator HFWVU - 2D, further developed and applied in this research, is based on PKN model only. This model has been selected because in the majority of low permeability unconventional reservoirs the fracture length is designed to be greater than fracture height, thus, creating larger fracture conductivity and reservoir contact area. Therefore, PKN model is a more practical choice to be applied for shale formation treatment designs.

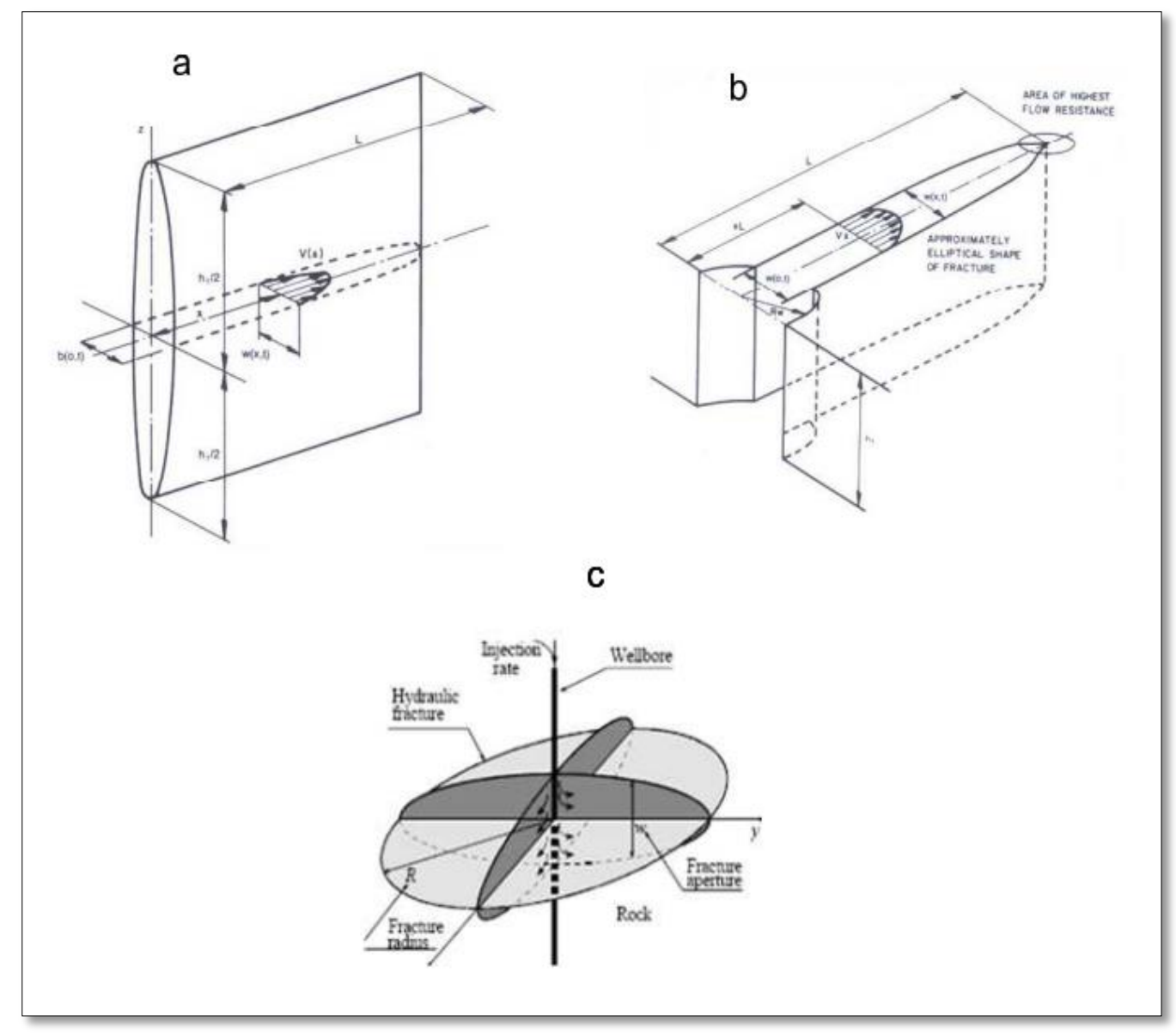

Figure 17 - Fracture geometry for a) PKN, b) GDK and c) Radial Models

(Michael J. Economides, Kenneth G. Nolte, 2000)

PKN and KGD 2D models have a few major assumptions:

1. The fracture is planar, and it propagates perpendicular to the minimum stress in a certain direction.

2. Fluid flow is one-dimensional in fracture length direction. 
3. Leak-off behavior is governed by an equation derived from filtration theory.

4. The fracture is confined, and in-situ stresses do not change horizontally.

5. Stresses occurring due to changes in reservoir pressure, temperature, and rock anisotropy are neglected.

\subsubsection{Pseudo-3D Models}

MFrac software also studied in this work, is not a fully 3-D model. It can be described "between a pseudo-3D and a fully 3-D type model", which has its half-length to a half-height ratio greater than 1/3. The 2D models were not able to simulate both horizontal and vertical crack propagation, and a pseudo-3D model was implemented by eliminating the assumption of a constant height. The pseudo-3D model calculates the height as the function of position along the fracture and time. In other words, 3D models are called 'pseudo' because they do not consider various fracture geometry in three-dimensional hydraulic fractures. Instead, they modify the 2-D PKN model by adding various height values along the fracture length and their effect on fracture width. The height can be either linear or parabolic. The major difference between the pseudo-3D and 2D models is an "additional vertical fluid flow component" and the major assumption is that "the fracture length is much greater than fracture height."

There are two main types of pseudo-3D models: the lumped and the cell-based models. The first lumped pseudo-3D model was introduced in 1986 by Settari and Cleary, and it assumes an elliptical geometry along the fracture length, and the fracture is symmetrical around the borehole. The cell-based model assumes fracture as a number of independently acting connected cells. Both pseudo-3D model types are limited to one-dimensional fluid flow assumption.

\subsubsection{Fully 3D Hydraulic Fracturing Simulators}

An accurate numerical simulation of fully 3D hydraulic fracturing involves solving two of the following problems:

1. An option to model the complex wellbore and fracture geometries. It includes geometrical and topological modeling tools with remeshing capabilities.

2. Solving a non-linear coupling between fluid flow equations and fracture propagation with deviation.

The second component includes stress analysis procedure, fluid flow simulation, and coupling the fracture propagation with fluid flow. B.J.Carter and J.Desroches (2010) offered a fully coupled 3D non-planar hydraulic fracture propagation code, called HYFRANC3D. The basis of the simulator was a coupled elasticity and lubrication theory, finite element solution based on this theory, and an iterative solution method. However, HYFRANC3D still treats hydraulic fracturing as a quasi-static process, where the solution is presented as series of "snapshots in time of the fracture geometry, fluid pressure, and crack opening displacements".

A fully 3D model is required to be coupled with full two-dimensional fluid flow. These models are based on LEFM studies coupling with the effects of complex fluid flow configurations along the fractures length (Hossain, 2001). The complex finite/boundary element methods are applied for fracture growth simulation using a mixed-mode fracture propagation criterion (for local stress-displacement field around the crack tip). The fracture can propagate laterally and vertically, and it might change the plane according 
to natural fractures/flaws direction, induced perforation, well inclination, local stress distribution and rock properties (Economides, 1994; Hossain, 2001). To the best of my knowledge, the code HYFRANC3D, being developed by the Cornell Fracture Group at Cornell University since 1987 (www.cfg.cornell.edu) (Martha, 1989; Wawrzynek, 1991; Potyondy, 1993), is the only hydraulic fracture analysis code that processes the mentioned above features. The code was written in Python, and it is designed for modeling arbitrary, non-planar, 3D cracks in complex structures with pre- and post-processing capabilities for both finite and boundary elements (Carter et al., 2000). While being more accurate, fully 3D modeling requires obtaining additional information on formation properties at the increased cost (Gidley, 1989) and it involves a high computational cost. In some cases, running one simulation with HYFRANC3D took over one month, and the fracture propagated up to $6 \mathrm{~cm}$ when using a high power computer (Hossain, 2001; Rahman, 2002). These simulations are time-consuming and not practical to be used, particularly for large repetitive calculations. However, these fully $3 \mathrm{D}$ models have a great value in academic research, where they serve as an important tool to understand all major aspects of hydraulic fracture propagation and diagnose some existing problems in hydraulic fracturing happening on the field.

The majority of simulators referred to as fully 3D models are still constrained to planar fracture surface. Planar-3D simulators, referred to in the literature as fully 3D simulators, have been developed by Clifton and Abou-Sayed (1979), Barree (1983), Touboul (1986), Morita (1988), Advani (1990). The first attempts to create fully 3-D non-planar models were made by Lam (1986), Vandamme and Jeffrey (1986), and Sousa (1993). However, Carter (1994) was the only one who modeled a fully 3-D non-planar hydraulic fracture in the near-wellbore area of a cased, perforated, and deviated wellbore.

\subsubsection{Classification of Commercial Simulators}

A wide range of hydraulic fracturing simulators is used in oil and gas industry nowadays. To be applicable for diverse cases, their models should be able to account for variable rock properties, reservoir properties, in situ stresses, fracturing fluids, and proppant loads. The most advanced fracture simulations include tip effects (MFrac handles it by using "over-pressure factor", which has to be obtained empirically, or by using great values of fracture toughness), wall roughness, complex fracturing, and some aspects of height growths (provided by Minifrac).

The SPE Forum compared hydraulic fracture models designed by twelve most common simulators, and they resulted with a difference up to 50\% while having the same input data (Warpinski et al., 1993). These models were categorized in the order of decreasing complexity as follows:

1) Planar three-dimensional (3D) models:

- TerraFrac of TerraTek, Inc. run by ARCO

- HYFRAC3D by Dr. Advani of Lehigh University

2) Unique Finite Difference Simulator GOHFER of Marathon Oil Co

3) Planar Pseudo-three-dimensional models

A. "Cell" Approach:

- STIMPLAN of NSI, Inc.

- ENERFRAC of Shell

- TRIFRAC of Holditch \& Assoc. 


\section{B. Overall Fracture Geometry Parameterization:}

- FRACPRO of RES, Inc

- MFRAC of Meyer and Assoc.

4) Classic PKN and GDK Models:

- PROP of Halliburton

- Chevron 2-D model

- Conoco 2-D model

- Shell 2-D model

- Pseudo-3-D models run in constant-height mode

The three main fracture simulation models used in the industry today are FracPro, FracproPT, and MFrac. FracPro and FracproPT are almost the same models, and they are based on either Cleary et al. approach or Meyer et al. approach (described Chapter 2, Section 2.3.3.). There is a hypothesis that FracPro and FracproPT models are more applicable for low permeability "hard" formations while MFrac models are more used for high permeability "soft" formations. The other simulators, such as StimPlan, Gopher and the simulators produced by Schlumberger, Halliburton, Shell and other companies are mostly used by engineers within these companies.

\subsubsection{Summary}

Linear Elastic Fracture Mechanics (LEFM) is a basis of the majority hydraulic fracturing simulators in oil and gas industry. It assumes elastic deformation followed by brittle fracture, with no energy utilized by non-linear deformation at the fracture tip. There were a few attempts to create models, different from the LEFM concept. However, they failed to receive widespread recognition for various reasons. It was discovered that for some formations the plastic deformation in the region around the fracture tip is not very significant. Besides, the formations above and under reservoir rock layer also have to be considered for the crack tip plasticity, since they can affect the fracture growth. Formations like soft carbonates, plastic shale, evaporate sand and any formation with a low yield stress and high fracture toughness are most likely to exhibit a significant level of plastic deformation. Plastic deformation may cause a difficulty to model accurately net pressure of low yield stress formations due to a considerable amount of fracture fluid energy, absorbed by a fracture tip. There is a hypothesis that MFrac is known in oil and gas industry for fracture modeling of "soft" formations, which are more likely to exhibit a significant level of plastic deformation with non-linear strain than "hard" formations. The software accounts for crack tip plasticity by adding a "tip over-pressure" effect. At the same time, the author of this fracture propagation model acknowledges that a "tip over-pressure" cannot be calculated. Therefore, this issue is handled by applying an "over-pressure factor", which has to be obtained empirically. However, the more detailed discussion in the above chapter shows, that an "over-pressure factor" is selected randomly by the user, until the simulation starts working properly. For this reason, there is still a big chance of achieving inaccurate results due to the effect of crack tip plasticity. 


\section{Chapter 3 - HFWVU-2D and MFrac Comparison and Limitations}

In this chapter, our in-house developed hydraulic fracturing simulator HFWVU-2D is compared with commercially available software MFrac, while both advantages and limitations of their numerical modeling and performance have been discussed and compared. MFrac was selected as an example of commercial hydraulic fracturing software, commonly used in the oil and gas industry for stimulation treatment design purposes. The provided below analysis gives an insight into numerical modeling of hydraulic fracturing, and it also serves as a tool for further understanding of fundamental fluid and solid mechanics governing the process of hydraulic fracturing.

\subsection{HFWVU - 2D Model}

HFWVU-2D model is a finite element based coupled method for numerical simulation of hydraulic fracturing propagation regimes, including two finite element systems as a part of its numerical model:

1. The first system was developed to find the relationship between the net pressure in the fracture and the fracture width.

2. The second system simulates the fluid flow in the fracture, which is non-linearly dependent on fracture width and linearly dependent on pressure gradient.

Simulation results were achieved by solving coupled equations from two mentioned above systems using Newton-Raphson iteration algorithm with accountancy for the energy dissipation regime and the storage regime. The energy dissipation regime ranges from toughness dominance to viscosity dominance while the storage regime ranges from zero leak-off to large leak-off. Both regimes depend on the material parameters of solid and fluid, and also on the fracture configuration.

Hydraulic fracturing regimes are controlled by a time-independent parameter $K_{m}$ (applied to distinguish the energy dissipation regime), and a time-dependent parameter $C_{m}$ under the assumption of LEFM for fracture propagation, lubrication theory for fluid flow, Carter's leak-off model, zero fluid lag between the fluid front and the fracture tip, constant injection rate, and large confining stress that leads to zero fluid lag (Lecamion, 2007).

HFWVU-2D simulator was developed based on a finite element method with a purpose to overcome limitations inherent to the boundary element method (Lecampion, 2009). Another common numerical method for hydraulic fracturing is known as displacement discontinuity (DD) method, and this boundary element method is used to model arbitrary shapes of fractures. It is based on the concept that one can make a discrete approximation to a continuous distribution of displacement discontinuity along a fracture. The formulation of DD method is based on Green's functions, which represent the relationship between displacement discontinuity and stress tensor. The fracture is divided into a series of $\boldsymbol{N}$ boundary elements, and the displacement discontinuity is considered constant over each one. Knowing the analytical solution for a single, constant elemental displacement discontinuity, the numerical solution of the problem is obtained by summing the effects of all $N$ elements.

A majority of finite element based models contain large size equations, dependant on the number of nodes in the whole model. Therefore, they are usually time-consuming and computationally costly. In contrast, 
HFWVU-2D has a built-in condensation technique for removing the node displacements that have no contribution to fracture width from the coupled equations. This condensation technique is independent of model remeshing techniques; it reduces the size of coupled equations greatly and results in the decrease of computational cost (Bao et al., 2014).

Another advantage of HFWVU-2D model is the opportunity to verify its validity by comparing numerical results with asymptotic results.

\subsubsection{Theoretical Model}

The relationship between fracture width $w$ and net fluid pressure $p$ at any moment $t$ in the half space $(x \geq 0)$ is computed with a following linear elastic fracture mechanics model:

$$
w(x, t)=\frac{\pi}{4 E^{\prime}} \int_{l_{t}} \ln \left|\frac{\sqrt{l_{t}^{2}-x^{2}}+\sqrt{l_{t}^{2}-x_{1}^{2}}}{\sqrt{l_{t}^{2}-x^{2}}-\sqrt{l_{t}^{2}-x_{1}^{2}}}\right| p\left(x_{1}, t\right) d x_{1}
$$

where $w(x, t)$ is fracture width at any point $x$ and time $t$ of fracture propagation, $l(t)$ is half fracture length at time $t$.

The fracture occurs when $K_{I}$ (intensity factor) reaches $K_{I C}$ (fracture toughness) at the fracture tip.

The governing equation for incompressible fluid flow in the fracture arises from the mass conservation of the fluid:

$$
\frac{\partial w}{\partial t}+\nabla \cdot q+g=0
$$

where $(\nabla \cdot)$ is divergence operator, $q$ is fluid flux, and $g$ is leak-off.

According to lubrication theory, the relationship between fluid flux $q$ and fracture width $w$ is given by Poiseuille law's i.e.,

$$
q=-\frac{w^{3}}{12 \mu} \nabla p_{f}
$$

where $\mu$ is fluid viscosity and $(\nabla)$ is gradient operator.

The leak-off is characterized by Carter's model as:

$$
g(x, t)=C_{l}^{\prime} / \sqrt{t-t_{0}(x)}, t>t_{0}(x)
$$

where $t_{0}$ is the fracture tip arrival time. If there is no fluid lag and the confining stress is uniform, the following equation can be applied:

$$
q=-\frac{w^{3}}{12 \mu} \nabla p
$$


The boundary conditions for fluid flow are the following:

$$
q\left(x=0^{+}, t\right)=\frac{Q_{o}}{2}, q(x=l(t), t)=0
$$

The first boundary condition in Equation (59) defines the symmetry of the fracture. The zero flux in the second boundary condition from Equation (59) is equivalent to zero width at the tip, also evident from Equation (58).

\subsubsection{Energy Dissipation Regimes}

Different hydraulic fracturing regimes can be defined based on the information about fluid storage and energy consumption in hydraulic fracturing (discussed in Chapter 3, Section 3.1), and they have been shown as a rectangular phase diagram $M \widetilde{M} K \widetilde{K}$ (Figure 18). The diagram displays two competing energy dissipation mechanisms changing in the vertical direction of the diagram, which are viscosity energy dissipation $(\mu)$ for fluid flow and toughness energy dissipation $(K)$ for fracture propagation. There are also two competing fluid storage mechanisms varying in the horizontal direction, which are fluid storage in the fracture and fluid leak-off $\left(C_{l}\right)$.

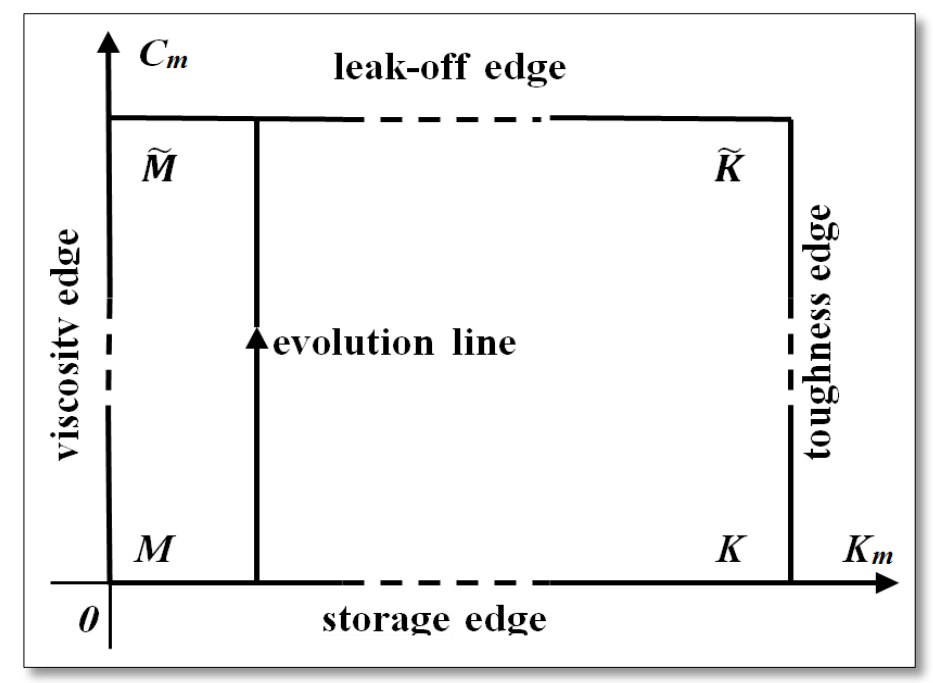

Figure 18 - Hydraulic fracturing regimes in the plane strain model

To quantify the regimes from the phase diagram, the analytical solutions can be characterized using dimensionless parameters $K_{m}$ and $C_{m}$ for a constant injection rate (described in Chapter 3, Section 3.1), which are defined as:

$$
K_{m}=\frac{K^{\prime}}{E^{\prime}}\left(\frac{E^{\prime}}{\mu^{\prime} Q_{0}}\right)^{\frac{1}{4}}, C_{m}=C^{\prime}\left(\frac{E^{\prime} t}{\mu^{\prime} Q_{0}^{3}}\right)^{\frac{1}{6}}
$$

$K_{m}$ characterizes the energy dissipation regimes, and it is time independent, while $C_{m}$ represents fluid storage regimes, and it is a time dependent parameter. The evolution line on the phase diagram shows a direction and a level of the leak-off increase into the formation. The leak-off coefficient is starting from 
zero leak-off at the early stage of fluid injection, when the injection efficiency is still equal to 1.0, and it evolves to infinity as the fluid loss is increasing due to natural fractures network.

Based on the $K_{m}$ value, hydraulic fracturing propagation regimes were classified into the toughnessdominated $\left(K_{m}>4\right)$, the intermediate $\left(1 \leq K_{m} \leq 4\right)$, and the viscosity-dominated ones $\left(K_{m}<1\right)$. They were further classified according to $C_{m}$ and compared with numerical models (Bao et al., 2014).

The phase diagram has four vertices and four edges, and each edge and vertex has different physical meaning. There are four edges, such as viscosity dominated, leak-off dominated, storage dominated and toughness dominated, and the physical processes characterizing each edge are described in Table 3. The physical meaning of each vertex can be understood by combining the meanings of intersecting edges. For example, $\widetilde{K}$ vertex in the phase diagram shows that all energy has been used for fracture propagation (toughness-dominated), and all the fracturing fluid is leaking into the formation.

Table 3 - Physical processes of the phase diagram

\begin{tabular}{|c|c|c|}
\hline Phase Diagram Edge & Meaning & Physical Process \\
\hline$M K$ & storage edge & Fracture propagation through an impermeable formation with zero leak-off \\
leak-off edge & High leakage of fracturing fluid into the formation \\
$M \widetilde{M}$ & viscosity edge & The fracture propagates along a pre-existing discontinuity \\
$K \widetilde{K}$ & toughness edge & The injected fluid has zero fluid viscosity, and all energy is used for \\
& & \begin{tabular}{c} 
fracture propagation \\
\hline
\end{tabular}
\end{tabular}

HFWVU simulator can be used to simulate hydraulic fracturing in all different regimes from the phase diagram, and simulations results have been compared with semi-analytical solutions in various fracturing regimes (Bao et al., 2014). The following section of this chapter gives more insight into the procedure of coupling solid medium response with fluid flow governing equations in the model.

\subsubsection{Finite Element Method}

\section{Elastic response}

A discretized linear relationship between net pressure increment and fracture width increment at every step is described as follows:

$$
\Delta w_{i}=\sum_{j} T_{i j}\left(\Delta p_{j}\right)
$$

where $\left(\Delta w_{i}\right)$ is the fracture width increment at nodes $i^{+}$and $i^{-}, \Delta p_{j}$ is the increment of net pressure applied on nodes $j^{+}$and $j^{-}$, and $T_{i j}$ is explained as the fracture width at point $i$ that is induced by unit pressure applied on nodes $j^{+}$and $j^{-}$. The matrix form of this equation is:

$$
\Delta w=T \Delta p
$$

where $(\Delta w)$ is the vector form of fracture width increment, $(\Delta p)$ is the vector form of net fluid pressure increment, and $T$ is the matrix form of $T_{i j}$ and is named as pressure influence coefficient matrix. 
Calculation of stress intensity factor:

$$
K_{I}=\frac{E^{\prime}}{2} \int_{A}\left[\sigma_{i j} \frac{\partial u_{i}^{a}}{\partial x_{1}}+\sigma_{i j}^{a} \frac{\partial u_{i}}{\partial x_{1}}-\sigma_{m n}^{a} \varepsilon_{m n} \delta_{1 j}\right] \frac{\partial x}{\partial x_{j}} d S-\int_{L} X P \frac{\partial u_{i}^{a}}{\partial x_{1}} d L
$$

where domain $A$ is a set of elements around the fracture tip, $L$ is a set of edges of finite elements in the set $A$ and these edges coincide with the fracture surface, $\sigma_{i j}\left(\sigma_{m n}\right)$ is a stress field, $u_{i}$ is a displacement field, $x_{j}(j=1,2)$ is a local coordinate, $\delta$ is the Kronecker operator, $X$ is a scalar field, and $\sigma_{i j}^{a}$ and $u_{i}^{a}$ are an auxiliary stress and displacement field, respectively.

\section{Fluid flow in the fracture}

According to the principle of variation and the divergence theorem, Equation (55) leads to the following form:

$$
\int_{l_{t}}\left[-\nabla(\delta p) \cdot q+(\delta p) \frac{\partial w}{\partial t}+(\delta p) g\right] d l+\left.\delta p\left(q \cdot n_{1}\right)\right|_{s}=0
$$

where $S$ is the collection of boundary conditions.

After combining the equations, fluid flow in the fracture is shown with the following equation:

$$
F(w, \dot{w}, p)=0
$$

Temporal integration of this equation between $\left[t_{n}, t_{n+1}\right]$ at the $n+1$ step leads to:

$$
\int_{t_{n}}^{t_{n+1}} F(w, \dot{w}, p) d t=0
$$

Backward Euler scheme for time difference is used in this paper, and Equation (66) yields:

$$
G\left(\Delta w, \Delta p ; p_{n}, w_{n}\right)=0
$$

where $w_{n}$ and $p_{n}$ are the unknown fracture width and fluid pressure at the $n-t h$ step.

The Newton-Raphson iteration algorithm is used to solve the non-linear coupling equations, where we have two unknown variables $w_{n}$ and $p_{n}$ :

$$
\begin{gathered}
\Delta w=T \Delta p \\
G\left(\Delta w, \Delta p ; p_{n}, w_{n}\right)=0
\end{gathered}
$$

The flow chart in Figure 19 shows a summary of the model workflow, displaying how solid medium and fluid flow equations are coupled and computed in the program to provide updated values of pressure and fracture width for each time step. The model description starts with boundary conditions (Equation 59) from the symmetry of biwing fracture and the zero fracture width at the fracture tip. The fluid flow in the 
fracture is modeled with using lubrication theory, and the relationship between fluid flux $q$ and fracture width $w$ is given by Poiseuille law's (Equation 56). Leak-off is characterized with time and geometry dependent Carter's model (Equation 57). The governing equation for incompressible fluid flow in the fracture arises from the mass conservation of the fluid (Equation 55), and it includes fracture width as a function of time, fluid flux as a function of solid medium deformation, and the leak-off of fracturing fluid into the formation. Then, based on the LEFM model, fracture width is computed as a function of pressure and time (Equation 54), and the calculations are performed though the M-integral. The model is discretized with finite element method, and each element has its latest solution, with fracture width and pressure values at time step $(\mathrm{n}+1)$ available after the Newton-Raphson Iteration Method.

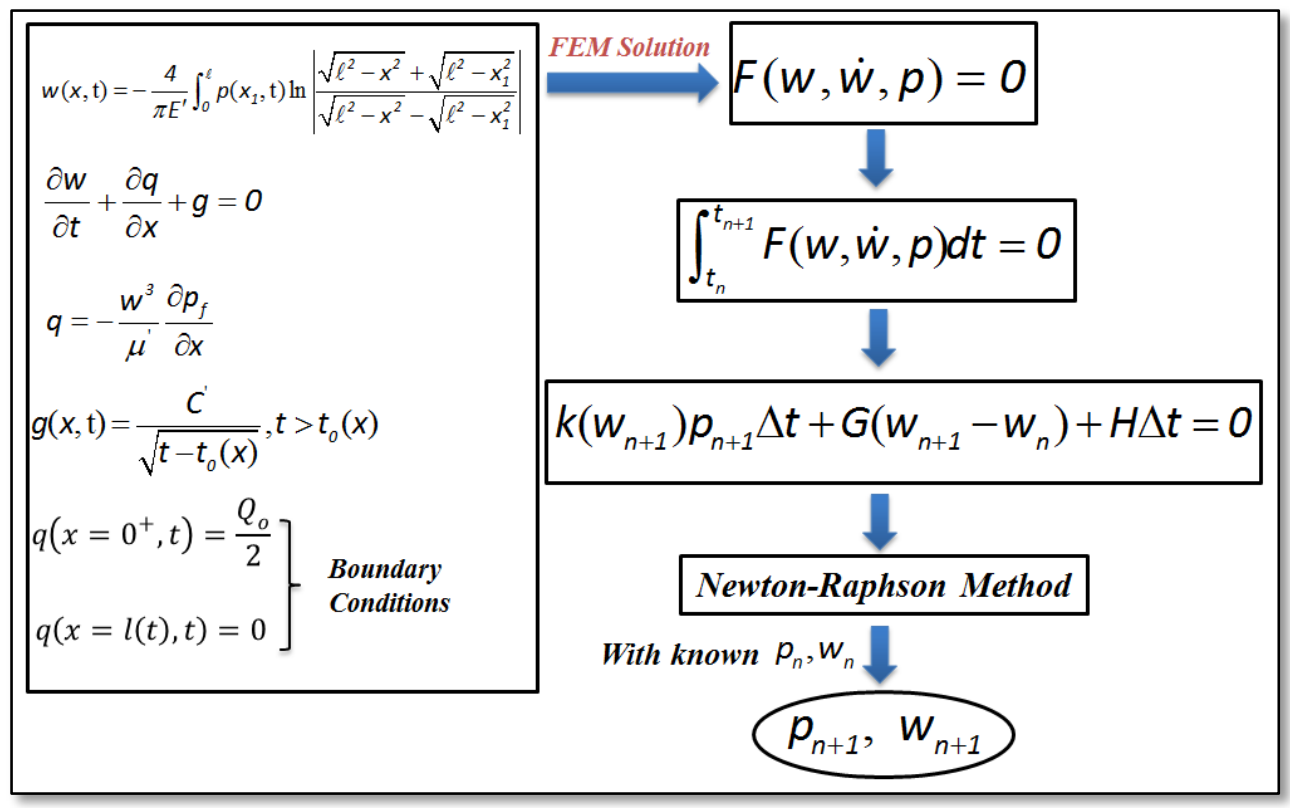

Figure 19 - Flow chart of HFWVU-2D workflow

\subsubsection{HFWVU-2D Results}

The numerical results of HFWVU-2D model were compared to some of the asymptotic solutions. Three cases covering the toughness-dominated, the intermediate, and the viscosity-dominated regimes with zero leak-off and with non-zero leak-off were reviewed. The results show that leak-off has limited effect on changing the tip behavior when the energy dissipation regime is toughness-dominated while it affects the tip behavior when the energy dissipation regime is viscosity-dominated (Bao et al., 2014).

\subsection{MFrac}

This section of the chapter offers an overview of the commercial software MFrac, which was selected as commonly applied in oil and industry representative of the simulators for hydraulic fracturing treatment design. This software is introduced as a candidate to compare our in-house model with. The comparison gives an insight into the differences in numerical modeling between our in-house HFWVU-2D simulator and a majority of commercial simulators, which often do not offer fully coupled fluid and solid mechanics governing equations. The comparison was made with a purpose to distinguish commercially available 
simulators, capable of providing a realistic representation of stress interference without discounting complex model features, necessary for this research study, or any other studies associated with the dynamic assessment of induced stresses and in-situ stress reorientation during multi-stage hydraulic fracturing in unconventional reservoirs.

\subsubsection{MFrac Modeling}

MFrac is a design and evaluation commercial simulator developed by Meyer, which offers a myriad of options for three-dimensional fracture geometry with coupled parameters influencing fracture propagation (including proppant transport and heat transfer routines). The software is a real-time and replay fracture multi-stage and multi-cluster simulation engine, used for predicting a complex fracture behavior in unconventional resources. MFrac takes into consideration all key parameters and provides an optimized multistage and multi-cluster method of hydraulic fracture treatments in horizontal wellbores. However, MFrac is not a fully 3-D model. Rather, it can be classified between a pseudo-3D and a full 3-D type model, which has its half-length to a half-height ratio greater than 1/3(Meyer \& Associates, 2015).

The premise of mechanical fracture interaction is that there are a number of parallel or dendritic fractures, which may or may not interact. MFrac assumes that the fracture system consists of $N$ similar fractures. The interaction functions and level of interaction are expressed in governing equations by applying the interaction factors $\psi$ and $\Phi$. MFrac models fractures separately for each multilayer fracture, then it illustrates the interaction between fractures by applying the interaction factors for flow rate, elastic stiffness and fluid flow. The independent interaction functions and levels of interactions are presented in the equations below:

\section{Flow Rate:}

$$
Q_{i}=\psi_{q} Q_{T}
$$

where

$$
\psi_{q}=\frac{1}{N}
$$

Stiffness:

$$
E_{i}=\psi_{E} E_{o}
$$

where

$$
\psi_{E}=(N-1) \Phi_{E}+1
$$

Fluid Loss:

$$
V_{i}=\frac{V_{T}}{N}
$$

where 


$$
\begin{gathered}
V_{T}=\psi_{l} V_{o} \\
\psi_{l}=(1-N) \Phi_{l}+N
\end{gathered}
$$

The user inputs the value of $\Phi$. The parameters with a subscript $i$ are individual fracture properties. The subscript $T$ gives the total value for $N$ fractures.

In case if fractures are not interacting, the values of $\Phi$ for stiffness and fluid are zero, and the value $\Phi$ is equal to unity in case of full interaction.

The following effects are not modeled in fracture pressure analysis, but they are considered by the simulator: multilayer leak-off, time-dependent fluid rheology, temperature influence on fluid rheology, variable formation properties, variable injection rates, fracture tip effects (dilatancy and fracture toughness), height growth in multi-stress zones, coupled fracture propagation, heat transfer and proppant transport, consistency in the fracture pressure analysis and treatment design, wall roughness and waviness (Meyer \& Associates, 2015).

\subsubsection{Governing Equations}

MFrac offers a solution method based on the coupled rock and fluid mechanics governing fracture propagation equations. The main parameters considered in the equations are: (1) multilayer unsymmetrical confining stress contrast, (2) multilayer leakoff, (3) fracture toughness and dilatancy (tip effects), (4) variable injection rate and time-dependent fluid rheology, (5) vertical and lateral rock deformation, (6) wall roughness and (7) coupled proppant transport, heat transfer, and fracture propagation.

The non-linear partial differential equations are solved with integral methods, and their description is provided further in this section.

\section{$\underline{\text { Mass Conservation }}$}

The fundamentals of mass conservation are based on overall mass balance for fluid flow into the casing and fracture system:

$$
\frac{\partial \rho V}{\partial t}=P Q_{\text {in }}-\sum_{i=1}^{n} P_{i} Q_{i}
$$

The above equation could have the following interpretation: the rate of mass accumulation in the casing (below the reference point) is equal to the rate of mass injected into the system minus the sum of the rates of mass out of the control volume into the individual fracture intervals. $Q i$ is into the fracture for positive flow and out of the fracture for negative flow during flow-back.

Fluid density in the control volume is assumed to be constant, where there is a same density in the casing and at the entrance to each fracture layer. Hence, the above equation can be simplified as follows, and it can be used for compressible and changing density slurries with time. 


$$
Q=\sum_{i=1}^{n} Q_{i}
$$

For consideration of wellbore storage and recirculation:

$$
Q_{t}=Q_{i n}-\frac{\partial V}{\partial t}
$$

\section{Continuity}

The mass continuity equation in terms of the flow rate per unit length $q=v * W$ is:

$$
\nabla * q+2 q_{L}+\frac{\partial W}{\partial t}=0
$$

where $\nabla * q=\frac{\partial q_{x}}{\partial x}+\frac{\partial q_{z}}{\partial z}$ and $q_{L}$ is the leak-off rate per unit leak-off area (for example, leak-off velocity), and $W$ is fracture width.

\section{$\underline{\text { Momentum Conservation }}$}

The equation of motion or momentum conservation equation for steady flow is:

where

$$
\nabla P=-\frac{1}{2} f \rho q^{-2} / w^{3}
$$

$f$ is the Darcy friction factor, Re is the Reynolds Number and $\varepsilon$ is the relative wall roughness.

\section{Width-Opening Pressure Elasticity Condition}

The crack-opening and opening pressure relationship is:

$$
W(x, z, t)=\Gamma_{W}(x, y, z, t) \frac{2(1-v)}{G} H_{\xi} \Delta P(x, 0, t)
$$

where $x$ - lateral coordinate along fracture length

$y$ - coordinate perpendicular to the frac face

$\mathrm{z}$ - coordinate parallel to the frac face

$\Gamma_{W}$ - a generalized influence function

$H_{\xi}$ - a characteristic half-height

$\Delta P$ - the net fracture pressure $P-\sigma$ (minimum horizontal stress) 


\section{Fracture Propagation Criteria}

The fracture criterion $K_{I C}$ states that crack propagation happens when $K_{I}=K_{I C}$. The stress intensity factor $K_{I}$ leads to crack growth, and it is a measure of fracture toughness (material property), which is the resistance force to crack extension.

$$
K_{I}=\sigma \sqrt{\pi} a
$$

where $\sigma$-elastic stress

$a$ - crack length

The critical value of $K_{I}$ (fracture toughness) can be calculated at the fracture stress when the crack length reaches a critical or maximum value before the rapid crack growth occurs. In MFrac model, the stress intensity factor equals the fracture toughness $K_{I}=K_{I C}$ or critical stress intensity of the rock $\sigma_{I}=\sigma_{I C}$. The simulator applies the greater of two values (Meyer \& Associates, 2015).

\subsection{MFrac versus HFWVU-2D}

\subsubsection{Simulators Characteristics}

\section{Model}

Hydraulic fracturing designs are usually performed by using in-house or commercial software, that is based on either KGD $(\mathrm{L} / \mathrm{H}<1)$ or PKN $(\mathrm{L} / \mathrm{H}>1)$ 2-D model. Less often, the software has both options available, and it chooses the most applicable one for a particular simulation. MFrac includes both PKN and GDK 2-D models, and there is also Penny shape 3-D model (Sneddon Model) included. However, HFWVU-2D simulator automatically chooses PKN model for all simulations. HFWVU-2D was simplified by eliminating the choices of 2-D models based on geometry because in hydraulic fracturing of unconventional reservoirs the fracture length is always greater than the fracture height. In contrast, KGD model is catering to higher permeability reservoirs.

\section{$\underline{\text { Regimes }}$}

Both MFrac and HFWVU-2D simulators consist of a range of hydraulic fracturing propagation regimes, obtained numerically for viscosity-dominated and toughness-dominated cases.

\section{Fluid Lag}

HFWVU-2D simulator allows modeling zero fluid lag, constant fluid lag, and dynamic fluid lag. To the best of my knowledge, all MFrac sources dismiss any type of discussion on fluid lag existence.

\section{Fracture Tip Effect}

HFWVU-2D model doesn't include the fracture tip behavior since its numerical method is irrelevant to the energy dissipation regime and fluid storage regime. In contrast, MFrac software includes a fracture tip effect that is described as "dilatancy" by the researchers. The term could be referred either to rock dilatancy or to fluid dilatancy. Rock dilatancy defines volumetric expansion of a material approaching the micro-cracking process. Fluid dilatancy describes shear-thickening fluid. Net pressure increase can be 
reached due to stress-dependent rock properties (could be related to rock dilatancy) or it can be achieved as a result of viscosity effects (fluid dilatancy). This process is described as a non-linear elastic deformation, and Figure 20 below illustrates its possibility.

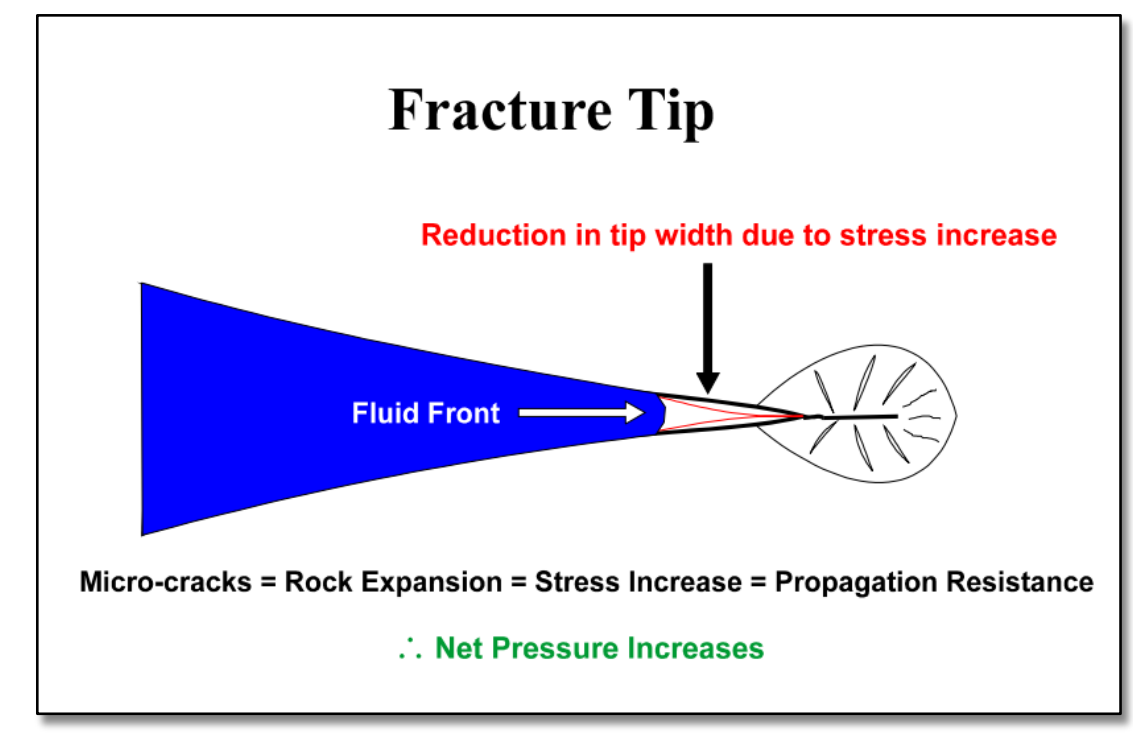

Figure 20 - Fracture tip width reduction due to non-linear elastic effects (Meyer \& Associates, 2015)

MFrac uses the term "tip over-pressure" that accounts for additional pressure required for fracture propagation. This pressure is necessary due to extra resistance at the tip. This parameter calculates additional pressure losses that occur in the fracture and have to be applied at the surface or BHTP. Tip behavior discussion still faces controversy with different explanations for these effects. Some researchers state that tip friction happens due to flow resistance and rock properties effects while others believe in it as a consequence of fracture geometry. Regardless which version is correct, tip friction still has the same effect on pressure (resistance or resilience). However, over-pressure is a function of injection rate and time and thus differs from other resilience, such as fracture toughness.

MFrac applies the range of the over-pressure factor between 0 and 1.0. The default value of zero is used in case if this option is disabled. The Tip Effect option is considered when measured injection pressures have a large discrepancy with theoretical values predicted by LEFM or other classic model.

After putting in the values for wall roughness, friction factor multiplier, toughness and other formation properties, a value between 0.1 and 0.4 can be corrected. When getting a large value of the over-pressure factor, the net pressure has to be increased accordingly. MiniFrac gives the option to decrease automatically the over-pressure factor to select the best-fit value. After matching the closest value, the net pressure value from minifrac analysis can be used for a further simulation.

\section{Time Dependent Fluid Rheology}

In contrast to HFWVU-2D simulator, MFrac includes non-steady injection rates, time-dependent fluid rheology, fracture toughness and spurt loss. First, the fracture propagation solution is achieved by a numerical method with meeting the requirements for mass conservation, continuity, momentum, elasticity relationship, and the fracture propagation criteria. After differential equations for fracture propagation are 
differentiated with respect to time, software applies the parameter $\boldsymbol{\alpha}_{\text {term }}$. It is described in MFrac user's guide as a parameter which accounts for the time dependent gamma parameters, various injection rates and fluid rheology, spurt loss, fracture toughness, etc. However, no supportive information as well as references displaying the calculations of $\boldsymbol{\alpha}_{\text {term }}$ and its effect on mentioned above parameters was provided.

\subsubsection{Governing Equations}

\section{Fracture Propagation Criteria}

The fracture criterion $K_{I C}$ states that crack propagation occurs when the condition $K_{I}=K_{I C}$ is met. The stress intensity factor $K_{l}$ leads to crack growth, and it is a measure of fracture toughness (material property), which is the resistance force to crack extension, Equation 86. The critical value of $K_{I}$ (fracture toughness) can be calculated at the fracture stress, when the crack length reaches a critical or maximum value before the rapid crack growth occurs. This is a definition of the mode-I dominated crack propagation (Figure 21) during hydraulic fracturing, applied in both simulators, besides the fact that in MFrac design the stress intensity factor equals the fracture toughness $K_{I}=K_{I C}$ or critical stress intensity of the rock $\sigma_{I}=\sigma_{I C}$. The simulator applies the greater of two values.

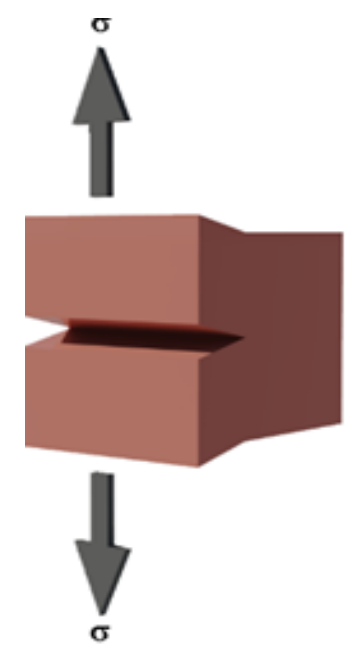

Figure 21 - Mode-I dominated crack propagation

\section{Mass Conservation}

The governing mass conservation equation for 'incompressible' slurry in the fracture is described as the total volume of slurry injected minus the volume of slurry in the fracture $\left(V_{f}\right)$ and the volume of fluid loss to the formation by leak-off $\left(V_{l}\right)$ and spurt loss $\left(V_{s p}\right)$ is equal to zero:

$$
\int_{0}^{t} q(\tau) d \tau-V_{f}(t)-V_{l}(t)-V_{s p}(t)=0
$$

where 


$$
\begin{gathered}
V_{f}(t)=r_{V}(t) W_{W}(t) H_{W} L(t) ; G D K \\
V_{f}(t)=r_{V}(t) W_{W}(t) H_{W} L(t) ; P K N \\
V_{f}(t)=2 r_{V}(t) W_{W}(t) R(t)^{2} ; \text { Penny } \\
V_{l}(t)=2 \int_{0}^{t} \int_{0}^{A} \frac{C(a, t)}{\sqrt{t}-\tau(A)} d A d t \\
V_{s p}(t)=2 S_{p} A(t) \\
\tau(A)=t\left[\frac{A}{A(t)}\right]^{\alpha_{a}}
\end{gathered}
$$

The above equations are solved numerically in MFrac.

In HFWVU-2D model the governing equation for incompressible fluid flow in the fracture is the following:

$$
\frac{\partial W}{\partial t}+\frac{\partial q}{\partial x}+g=0
$$

where $\nabla$ - divergence operator

$q$ - fluid flux

$g$ - the leak-off

Unlike MFrac, HFWVU-2D does not consider spurt loss $V_{s p}(t)$ in mass conservation governing equation. Spurt loss is a continuous volume loss of fluid per unit area of fracture face happening before a filter cake is formed, and usually occurring only in the pad volume. In case when the spurt time constant is of the same order as the pump time, we might have issues in modeling the total fluid loss and receiving incorrect data, resulted from modeling spurt loss as instantaneous mechanism with a linear leak-off. Due to uncertainties in spurt loss value and its possible effect on obtaining an incorrect total fluid loss, HFWVU-2D has eliminated spurt loss parameter from the mass conservation equation.

\section{$\underline{\text { Momentum Conservation }}$}

In MFrac software, the equation of motion or momentum conservation equation for steady flow is the following:

$$
\nabla P=-\frac{1}{2} f \rho q^{-2} / w^{3}
$$

where

$$
f=\frac{24}{R e} \text { for laminar flow }
$$




$$
f=f(R e, \varepsilon) \text { for turbulent flow }
$$

$f$ is the Darcy friction factor, Re is the Reynolds Number and $\varepsilon$ is the relative wall roughness.

The fluid flux arises from the above equation:

$$
q^{-2}=-\frac{2 \nabla P w^{3}}{f \rho}
$$

The definition of Reynold's Number is as follows:

$$
R_{e}=\frac{\rho v L}{\mu}
$$

Then, the Darcy friction factor is:

$$
f=\frac{24 \mu}{\rho v L}
$$

After simple calculations, the fluid flux is as follows:

$$
q^{-2}=-\frac{\nabla P w^{3} v L}{12 \mu}
$$

According to the HFWVU-2D model basics and lubrication theory, the relationship between the fluid

$$
q=-\frac{\nabla P w^{3}}{12 \mu}
$$

flux $q$ and fracture width $w$ is given by Poiseuille's law:

The momentum conservation equations should be the same in both simulators; however, there is a visible difference between them.

\section{Continuity}

MFrac is based on the mass continuity equation in terms of the flow rate per unit length $q=v * W$ :

$$
\nabla * q+2 q_{L}+\frac{\partial W}{\partial t}=0
$$

Where $\nabla * q=\frac{\partial q_{x}}{\partial x}+\frac{\partial q_{z}}{\partial z}, q_{L}$ is the leak-off rate per unit leak-off area (for example, leak-off velocity), and $W$ is the fracture width.

Likewise, in HFWVU-2D model the continuity equation, Equation 94, is presented as the governing equation for incompressible fluid flow in the fracture, which is absolutely identical with MFrac mass continuity governing equation. 


\section{Width-Opening Pressure Elasticity Condition}

Both MFrac and HFWVU-2D simulators describe the crack-opening and opening pressure relationship with an LEFM, Equations $54 \& 85$. Also, MFrac includes a generalized influence function $\Gamma_{W}$ in the model, Equation 85. MFrac refers to Perkins et al., 1961 for details about $\Gamma_{W}$ and gamma coefficients for 3-D fracture models. However, this source doesn't include mentioned above explanations. Therefore, it is not clear where the values originally come from.

\section{$\underline{\text { Boundary Conditions }}$}

According to the theory, each model should allow either flow rate or pressure boundary conditions. In HFWVU-2D model the initial injection rate is assumed to be equal zero. HFWVU-2D determines injection flow rate boundary conditions for fluid flow and displacement boundary conditions for the solid part with fully coupled equations. Contrary, MFrac assumes initial bottomhole pressure to be equal zero for calculating boundary conditions.

\section{HFWVU - 2D Model}

The boundary conditions for fluid flow:

$$
q\left(x=0^{+}, t\right)=\frac{Q_{0}}{2}, q\left(x=l_{t}, t\right)=0
$$

where $q$ is the fluid flux and $Q_{0}$ is the injection rate.

The first boundary condition is from the symmetry of the fracture. The second boundary condition of zero flux at the fracture tip comes from the governing equation for incompressible fluid flow:

$$
\frac{\partial w}{\partial t}+g=\nabla \cdot q
$$

where $(\nabla \cdot)$ is the divergence operator and $g$ is the leak-off. This equation shows that some of the injected fluid accommodates in the fracture and increases the fracture width, while some fluid leaks into the formation.

The second boundary condition of zero flux at the fracture tip is better seen in lubrication theory, where Poiseuille's law shows the relationship between the fluid flux $q$ and fracture width $w$, Equation 56.

The leak-off into the formation is described by Carter's, Equation 57.

The boundary conditions for solid part (Figure 22):

$$
\begin{gathered}
\sigma \cdot n=p_{f}(x, t), \quad x \in S_{f} \\
u_{x}=0 \text { at } x=0 \\
\sigma=\sigma_{0},|y|=\text { const }
\end{gathered}
$$


where $n$-the unit normal of the fracture

$u$ - displacement

$\sigma_{0}$ - confining stress

$S_{f}-$ fracture surface

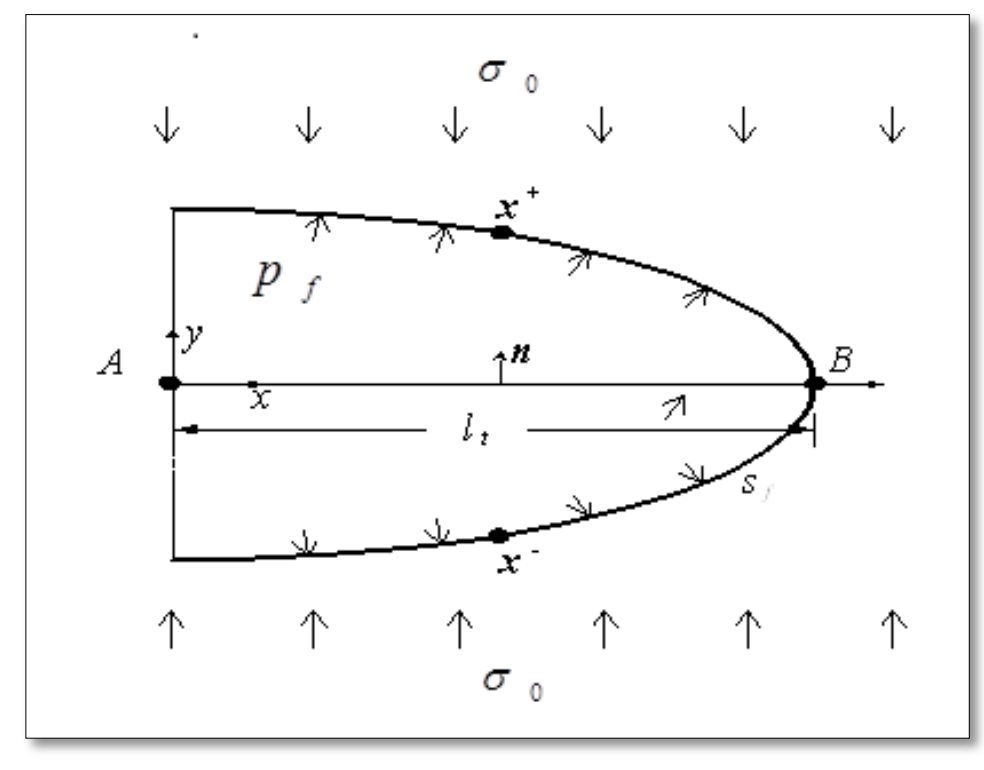

Figure 22 - Fracture profile

\section{MFrac}

The Data Options screen of MFrac has General Options dialogue, which allows the user to select the type of analysis to be performed. Reservoir coupling from this dialogue gives an option of control and flexibility for the fluid loss mechanism. MFrac offers a choice of two reservoir couplings: linear (conventional) and ellipsoidal (Koning).

Linear (Conventional) Reservoir Coupling is a basic type of fluid loss mechanism, where the rate of fluid loss to the solid medium is governed by a total leak-off coefficient $C$. It is also known as diffusion controlled fluid loss mechanism. This option can be used if the leak-off distance perpendicular to the fracture is much less than the fracture length. The characteristic leak-off distance at transition $\delta(t) \rightarrow \delta$ can be approximated from mass conservation of the particles when a large fraction of the particles are deposited in the internal cake.

Ellipsoidal (Koning) Reservoir Coupling is used for long periods of produced water reinjection or waterflood injection. This model is based on the work of Koning and his 2-D ellipsoidal fluid loss model. There are three boundary conditions for ellipsoidal fluid loss:

1) Closed System - no flow boundaries

2) Constant pressure boundary condition (B.C.) at the initial reservoir pressure

3) Pseudo-Steady State - the average reservoir pressure is equal to initial reservoir pressure. Pseudosteady behavior of reservoir means that the production rate is equal to the injection rate 
For After-Closure Analysis, MFrac offers linear solution, assuming one-dimensional fluid loss and the following boundary conditions:

1. Constant Velocity Boundary Condition - the boundary and initial conditions, assuming the reservoir to be infinite with a constant leak-off velocity $v_{0}$ :

$$
\begin{gathered}
p(y, 0)=p_{i} \text { (initial condition) } \\
v(0, t)=v_{0}=-\left.\frac{k}{\mu}\left(\frac{\partial p}{\partial y}\right)\right|_{y=0} \\
\text { for } t>0 \text { (constant leak }- \text { off velocity) }
\end{gathered}
$$

2. Constant Pressure Boundary Condition - the boundary and initial conditions, assuming the reservoir to be infinite with a constant pressure in the fracture between the fracture and the reservoir:

$$
\begin{gathered}
p(y, 0)=p_{i}(\text { initial condition }) \\
p(0, t)=p_{f} \\
\text { for } t>0(\text { pressure at inj.point is constant over } t)
\end{gathered}
$$

3. Time Dependent Velocity Boundary Condition - Nolte (1997) first presented a closed form solution for "the changed-boundary condition and an infinite-length fracture, or equivalently for linear flow from a fixed length fracture, the constant-pressure condition for pre-closure" followed by a zeroflux condition. Nolte offered a solution that was based on the study of Carslaw and Jaeger, and it's final form is presented in the following equation:

$$
\Delta p_{R}=v_{c} \sqrt{t_{c}} \sqrt{\frac{\pi \mu}{k c \Phi}}\left\{\frac{2}{\pi} \arcsin \theta^{-1 / 2}\right\}
$$

where $\Delta p_{R}=p_{R}-p_{i}$ is the pressure differential carried by the reservoir

$$
\begin{aligned}
& v_{c}=C_{T} / \sqrt{t_{c}}-\text { leak-off velocity at closure } \\
& F(\theta)=\frac{2}{\pi} \arcsin \theta^{-1 / 2}-\text { dimensionless linear time function }
\end{aligned}
$$

After-Closure Analysis - Linear Solution Summary. From more detailed Nolte's formulation of linear functions, the governing equation for the pressure solution for multiple injection rates was finalized as follows:

$$
p-p_{i}=m_{L} F_{L}\left(t, t_{c}\right)
$$

where $m_{L}$ is the pressure at closure when $(\theta=1)$, also equal to $\left(\mathrm{p}\left(\mathrm{t}_{\mathrm{c}}\right)-\mathrm{p}_{\mathrm{i}}\right)$, and it is described with a following equation:

$$
m_{L}=C_{T} \sqrt{\frac{\pi \mu}{k c_{t} \Phi}}
$$


$F_{L}$ is a dimensionless fluid loss time function, found as:

$$
F_{L}\left(t, t_{c}\right)=\sqrt{1+\left(t-t_{c}\right) / x t_{c}}-\sqrt{\left(t-t_{c}\right) / x t_{c}}
$$

where $x t_{c}$ is an apparent closure time defined by Nolte (1997), where $x=16 / \pi^{2}$

Since $F\left(t, t_{c}\right)$ is the same function from which $x$ was formulated, $F_{L}\left(t, t_{c}\right)$ can be solved as it is equal to $F\left(t, t_{c}\right)$ :

$$
F_{L}\left(t, t_{c}\right) \stackrel{\text { yields }}{\longrightarrow} F\left(t, t_{c}\right)=\frac{2}{\pi} \arcsin \sqrt{t_{c} / t}
$$




\section{Chapter 4 - Methodology}

\subsection{Identifying Transient Stress Behavior}

Stimulation design includes selecting the optimum fracture spacing, injection rate and volume, and proppants design. This study is focused on understanding the developing stress patterns surrounding fracture(s), and characterizing their behavior in order to adjust stimulation treatment plans in real time to mitigate the merging of fracture stages. The merging of fracturing stages is a persistent problem because of changes in the local stress field surrounding the hydraulic fractures in the real time. As a fracture propagates, the initial direction and magnitude of the surrounding stresses may change from their expected or initial values, which cause issues in subsequent fracture development process. These altered stress fields may hinder the growth of fracture development or cause the merging of fracture(s) or fracture stages, which drastically impacts the efficiency of the hydraulic fracture stimulation.

The objective of this research was met by considering different cases of single, two, and three fractures and quantifying stress variation around the induced hydraulic fractures through time by studying the unique behavior of Von Mises and principal stresses. Figure 23 describes the transient behavior of maximum principal and Von Mises stresses in the field. This plot illustrates both maximum principal stress and Von Mises stress depicting similar behavior through time, defining Von Mises stress as an applicable culmination of stresses for analyzing their transient patterns. Figure 23 shows four distinct stress behaviors, seen through the period of injection. At early time, the magnitude of the principal and Von Mises stresses increases rapidly as the injected fluid is starting breaking through the formation and there is a large stress accumulation around the initiated fracture. After analyzing various simulations, it was also observed that at the earlier time of fracture propagation there is a rapid deformation of the solid medium, and the model offers a larger number of solutions due to intensive fracture width and pressure updates values for each time step. Then, the increment between time steps is increasing, and the stress magnitude slows down as the fracture tip is moving away from injection point till it reaches its maximum value at later time, when the stress value at the fracture tip is still very high, and stress profiles at the fracture head are reaching their maximum values while the stress profiles at the fracture tail are also still high (Figure 24). At a later time, there is a decline in stress magnitude due to the injection fluid energy loss and a significant decay in stress values at the fracture tip with stress magnitude finally reaching a constant value at later injection time. Therefore, to avoid stress interference during sequential hydraulic fracturing, it important to monitor the transient principal and Von Mises stress behavior in the reservoir, and avoid initiating sequential fracture propagation until the stress magnitude in the reservoir, influenced by the previous fracture, reaches a steady state.

Figure 24 shows the maximum Von Mises stress in different cross sections of the 2D model perpendicular to the fracture plane as a function of the distance from the fracture tip. The plot displays consistent stress behavior with time both for early and later stages of fracture propagation. Figure 24 (a) and (b) show the early and late time responses where different colors represent different time steps. The maximum Von Mises stress always happens at the fracture tip. At early time, the maximum Von Mises stress is also influenced by injection point, i.e. located at different locations with respect to fracture tip at different time steps and presented with negative distance value, which leads to having the maximum Von Mises stress values higher at the fracture tail than distance ahead of the fracture tip. At a later time, the impact of injection point diminishes, and the tail and head of the fracture tip behave much alike. 


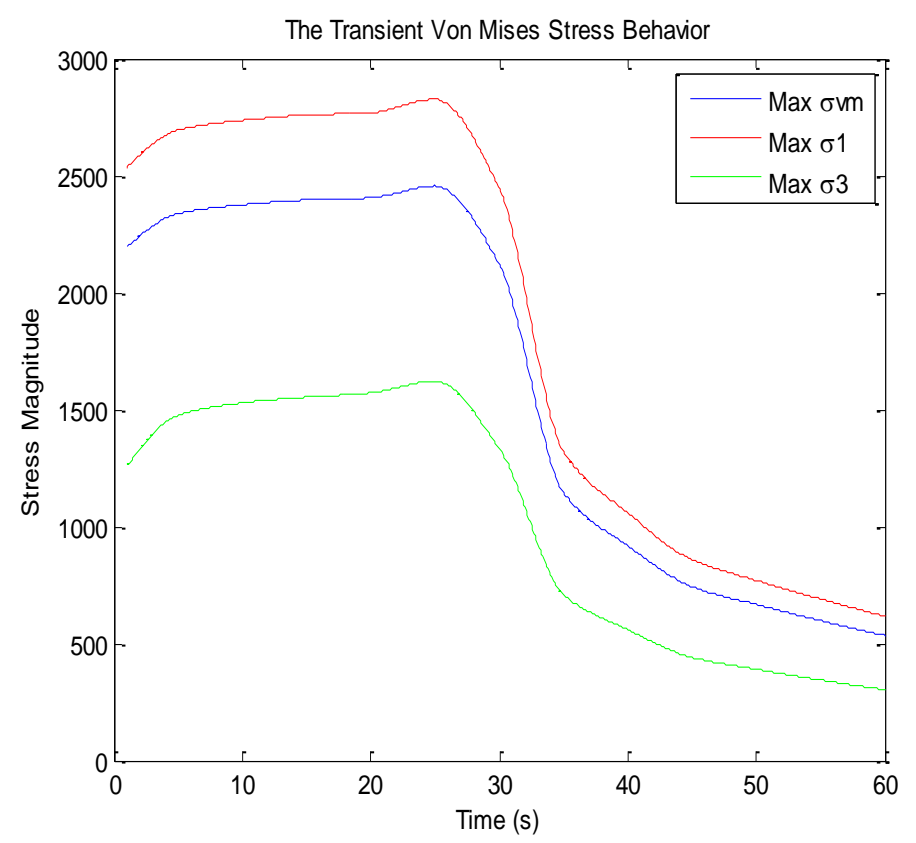

Figure 23 - The transient behavior of maximum principal and Von Mises stress variations surrounding a single fracture, $\mathrm{t}=\mathbf{0}$ to 60s
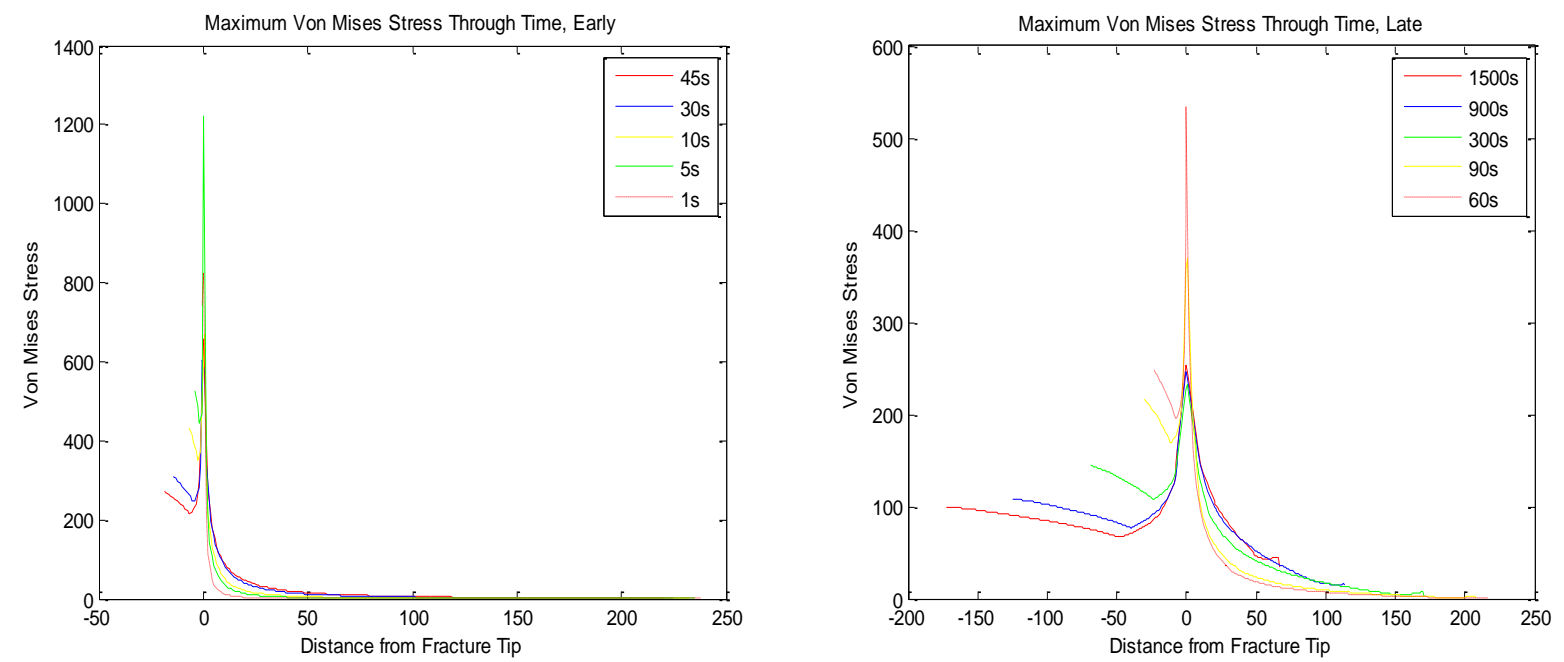

Figure 24 - Maximum Von Mises Stress with relation to the fracture length at distance from the fracture tip and (a) at early simulation time; (b) at late simulation time

\subsection{Critical Radius}

To quantify the critical radius, where a significant change in the state of field stress along propagating fracture occurs, the theory of critical distance (TCD) (Taylor, 2007) was applied and further extended in this study. The critical distances (TCD) found application in predicting fatigue and failure in a wide range of materials and components (Taylor, 2007; Yin et al., 2014; Louks et al., 2014). The TCD was also applied towards predicting fracture of rocks and minerals (Lajtai, 1971; Ito et al., 1991). 
The fracture toughness value for Marcellus shale formation, used in as an input parameter of my simulations $\left(1.77 \mathrm{MPa} \cdot \mathrm{m}^{1 / 2}\right)$, is matching to the value of quasi-brittle material from experimental studies in the literature $\left(1.7 \mathrm{MPa} \cdot \mathrm{m}^{1 / 2}\right.$ ) (Louks, et al., 2014). Based on the TCD, the following engineering value for the critical distance was applied for computing the distance with maximum probability of failure:

$$
D=\frac{1}{2 \pi}\left(\frac{K_{I C}}{\sigma_{V M}}\right)^{2}
$$

In above Equation, $(D)$ is distance to the point of investigation with the highest probability of failure, and $\left(\sigma_{V M}\right)$ is the maximum principal stress at the specific point of investigation. Rewriting the above Equation based on maximum Von Mises stress at the cross-section perpendicular to the fracture plain allows us to calculate the critical radius $(R)$ with high probability of failure when Von Mises stress reaches its maximum value:

$$
R=\frac{1}{\pi}\left(\frac{K_{I C}}{\sigma_{V M}}\right)^{2}
$$

In above equation, $\mathrm{K}_{\mathrm{IC}}$ is the fracture toughness that is a rock property and remains constant through time, leaving the critical radius to be dependent only on the dynamics of Von Mises stress:

$$
R=f\left(G_{V M}\right)
$$

Based on the summarized above observations, a new theory for quantifying the magnitude of stress variation in real time is developed and examined. An earlier identified stress pattern (Figure 24) was used in this study to provide a tool to investigate the transient behavior of stress at a different distance from the injection point. Four investigation points were primarily selected (Figure 25) to conduct this study and quantify the critical radius through time. Point 1 is an injection point, which has high-stress magnitude and the probability of formation failure. Point 2 is where the fracture tip effect starts dominating the injection point effect, and, therefore, can be used as the basis for a state of stress where an important change in stress magnitude and orientation occurs. Point 3 is a fracture tip with the highest probability of failure in surrounding area and point 4 is located at the head of the fracture where the change in stress is assumed to be not significant.

As presented in Equations 119 and 120, the critical distance has an inverse relation with principal or Von Mises stress. As the distance to fracture tip increases the magnitude of the stress decreases. Therefore, the critical radius at any point away from the fracture tip increases. However, there are different probabilities of failure associated with the critical radius calculated at different locations, where the highest probability of failure assigned at the fracture tip with smaller critical radius. To have a consistent measure of critical radius, the magnitude of stress at point 2 is picked, presented in Figure 26 at the end of the hydraulic fracturing as the point where we consider a significant change in magnitude and orientation of stress occurs. 
This point also had a unique characteristic showing the local minimum Von Mises stress throughout the simulation time and also in different hydraulic fracturing flow regimes, i.e. toughness dominated, intermediate and viscosity-dominated regimes. Using this technique, the probability of 1 was assigned to the critical radius calculated at point 2 at the end of hydraulic fracturing simulation, i.e., before model boundaries being influenced by a change in field stress, and the critical radii at different locations were scaled based on that. Table 5, covered in the Results Chapter, shows the details of stress and critical radius calculations at point 2 and fracture tip "point 3".

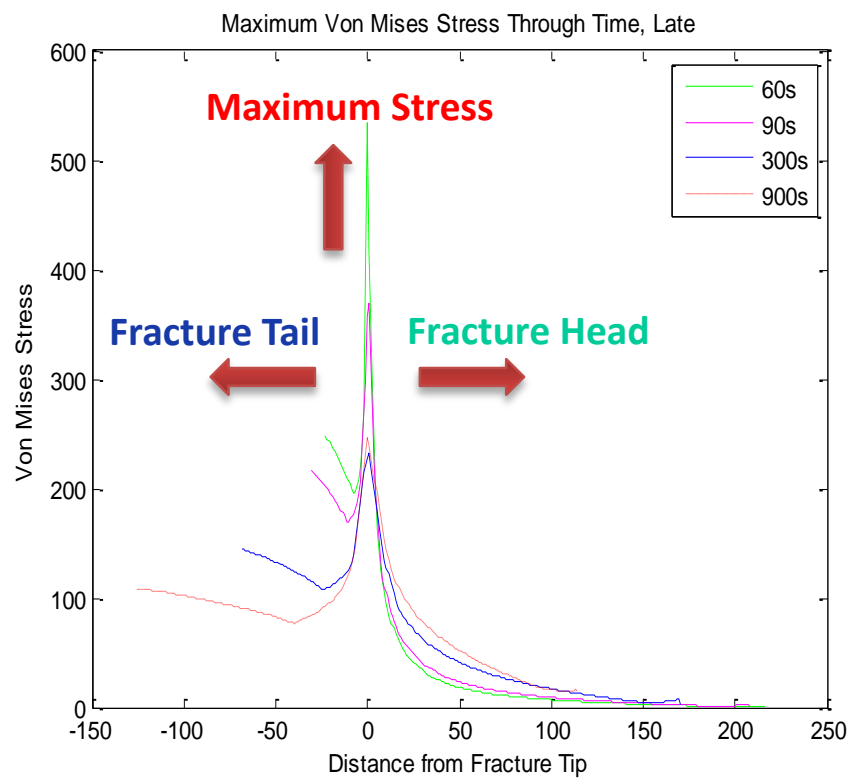

Figure 25 - Maximum Von Mises Stress with relation to the fracture length at distance from the fracture tip and at late simulation time

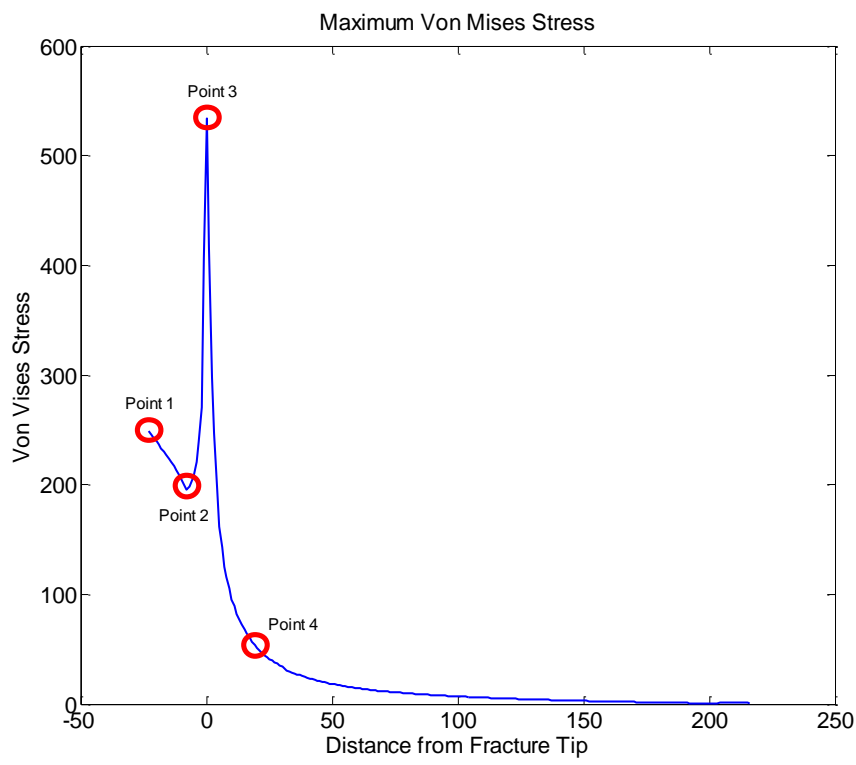

Figure 26 - Points of investigation for static Von Mises Stress Distribution 


\subsection{Flow Regimes}

Different hydraulic fracturing flow regimes show the different impact on magnitude and orientation of stress around hydraulic fractures. The major influence upon the stress profile is throughout the length of the fracture. Prior to the fracture tip, the flow regime controls the maximum stress and ratio of stress between the injection point and the fracture tip. Figure 27 (a) shows the viscosity-dominated regime with fracture toughness value of $0.25 \mathrm{MPa} \cdot \mathrm{m}^{1 / 2}$, that is causing a significant stress at the injection point, greater than that of the fracture tip stress. This injection point stress progressively declines as the tip stress raises at different flow regimes (large viscosity-intermediate and intermediate-large toughness). In the case of toughness-dominated flow regime with fracture toughness value of $4.00 \mathrm{MPa} \cdot \mathrm{m}^{1 / 2}$, the injection point shows the minimum impact. An intermediate flow regime with fracture toughness value of 1.77 $\mathrm{MPa} \cdot \mathrm{m}^{1 / 2}$ depicts a transitional stress behavior between the viscosity-dominated and the toughness-dominated regimes. Table 4 displays the characteristics of each flow regime described in the simulation results on Figure 27.

Table 4 - Flow Regime Cases

\begin{tabular}{|ll|}
\hline Flow Regime & $\mathbf{K}_{\mathbf{I C}}$ Value \\
\hline Viscosity-Dominated & $0.25 \mathrm{MPa} \cdot \mathrm{m}^{1 / 2}$ \\
Intermediate & $1.77 \mathrm{MPa} \cdot \mathrm{m}^{1 / 2}$ \\
Toughness-Dominated & $4.00 \mathrm{MPa} \cdot \mathrm{m}^{1 / 2}$ \\
\hline
\end{tabular}
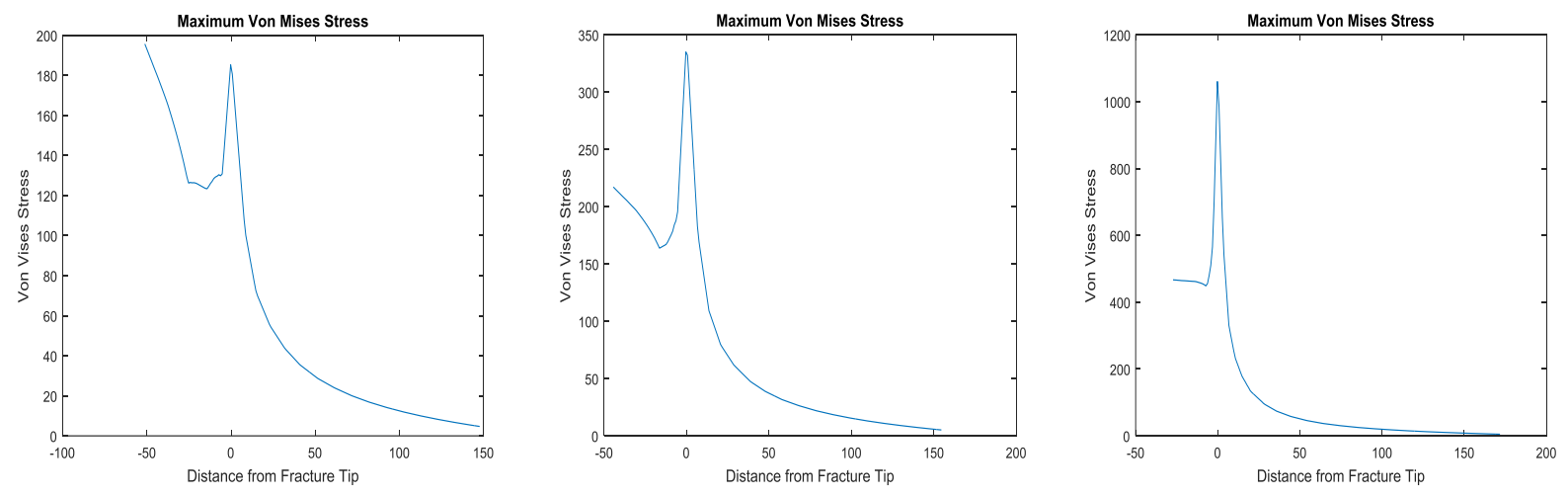

Figure 27 - Von Mises stress along the fracture half-length at equal snapshots in time, $t=60 \mathrm{~s}$ (a) large viscosity case, (b) intermediate case, (c) large toughness case 


\section{Chapter 5 - Results}

\subsection{Critical Radii for Single, Two and Three Fractures}

The critical radius at the fracture tip at the end of simulation is obtained to be $4.77 \mathrm{~m}$ using Equation 119 . The calculated critical distance is confirmed in Figure 28 considering the $169.3 \mathrm{KPa}$ as a basis for comparison of the significant change in the state of the stress at 90 seconds (Table 5). As shown in Table 5, the critical radius has the largest value at earlier time steps where the value of Von Mises stress is higher and shrinks as Von Mises stress declines. This is in contrary to Equation 119, because as stated earlier here the critical radius at different time steps has the same probability of failure unlike the original TCD, i.e., Equation 119 and 120, where at different time steps different probability of failure were assigned to the critical radius.

Table 5 displays an example of sequential fracturing, where the second fracture is initiated in the 90 s after the first fracture has started propagating. In this case, critical radii at point 3 (fracture tip), where the maximum Von Mises Stress occurs, is normalized by the value of maximum Von Mises Stress at point 2 at the $90 \mathrm{~s}(169.3 \mathrm{KPa})$.

Table 5 - Radii of failure for a single fracture case

\begin{tabular}{|c|c|c|c|c|}
\hline \multirow[b]{2}{*}{ Time, $\mathrm{s}$} & \multicolumn{2}{|c|}{ Point 2} & \multicolumn{2}{|c|}{ Point 3} \\
\hline & $\begin{array}{c}\text { Comparative } \\
\text { Probability } \\
\text { of Failure, } P\end{array}$ & Von Mises Stress, $\sigma_{\mathrm{VM}}(\mathrm{KPa})$ & Radius, R & Von Mises Stress, $\sigma_{\mathrm{VM}}(\mathrm{KPa})$ \\
\hline 10 & 4.25 & 349.2 & 15.60 & 668.7 \\
\hline 30 & 2.15 & 248.2 & 12.64 & 602 \\
\hline 45 & 1.62 & 215.8 & 15.00 & 655.7 \\
\hline 60 & 1.33 & 195.4 & 9.95 & 534 \\
\hline 90 & 1.00 & 169.3 & 4.77 & 369.8 \\
\hline
\end{tabular}

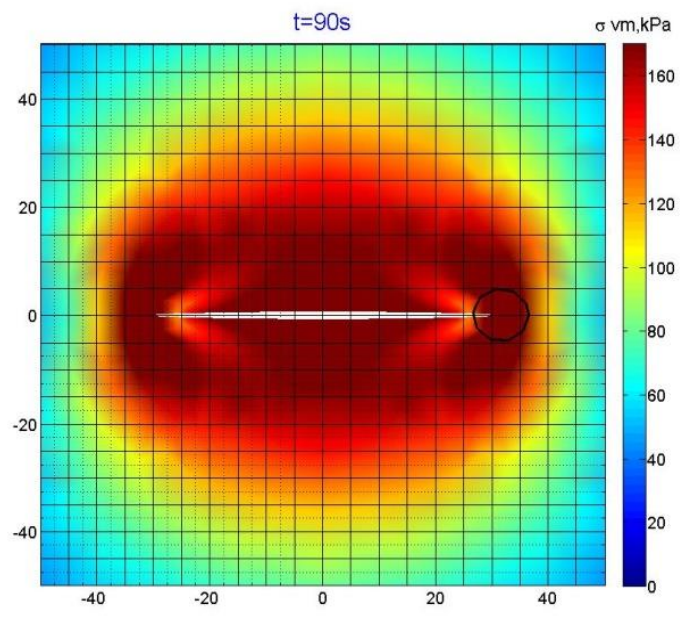

Figure 28 - Critical radius at the fracture tip after 90s of injection 
Because of minor fluctuations in critical radii values at the early time of fracture propagation, caused by injection point effect, I selected the first and the second largest critical radii, equal to $15.60 \mathrm{~m}$ and $12.64 \mathrm{~m}$, and corresponding optimum fracture spacing were approximately $25 \mathrm{~m}$ and $30 \mathrm{~m}$. However, for a higher level of confidence, considering the radius at the earliest time of fracture propagation should be sufficient.

Figure 29 confirms the critical distance, obtained from Table 5 after 10 seconds of simulation for single, two and three fractures cases. The given plots display the stress accumulation around 2 and 3 simultaneously propagating fractures at their earlier stage, when we usually observe the highest stress interaction. The plot with a 2 -fracture case is showing the scenario with $12.64 \mathrm{~m}$ critical radius and approximately $25 \mathrm{~m}$ distance between fractures. The interaction between fractures is still visible. However, when I considered the highest value of critical radius $(15 \mathrm{~m})$, and corresponding optimum fracture spacing of $30 \mathrm{~m}$, as it has been displayed on the plot with 3 fractures, there is no more meaningful interaction. Therefore, optimum fracture spacing has been predicted correctly, and propped fractures would produce successfully without merging.
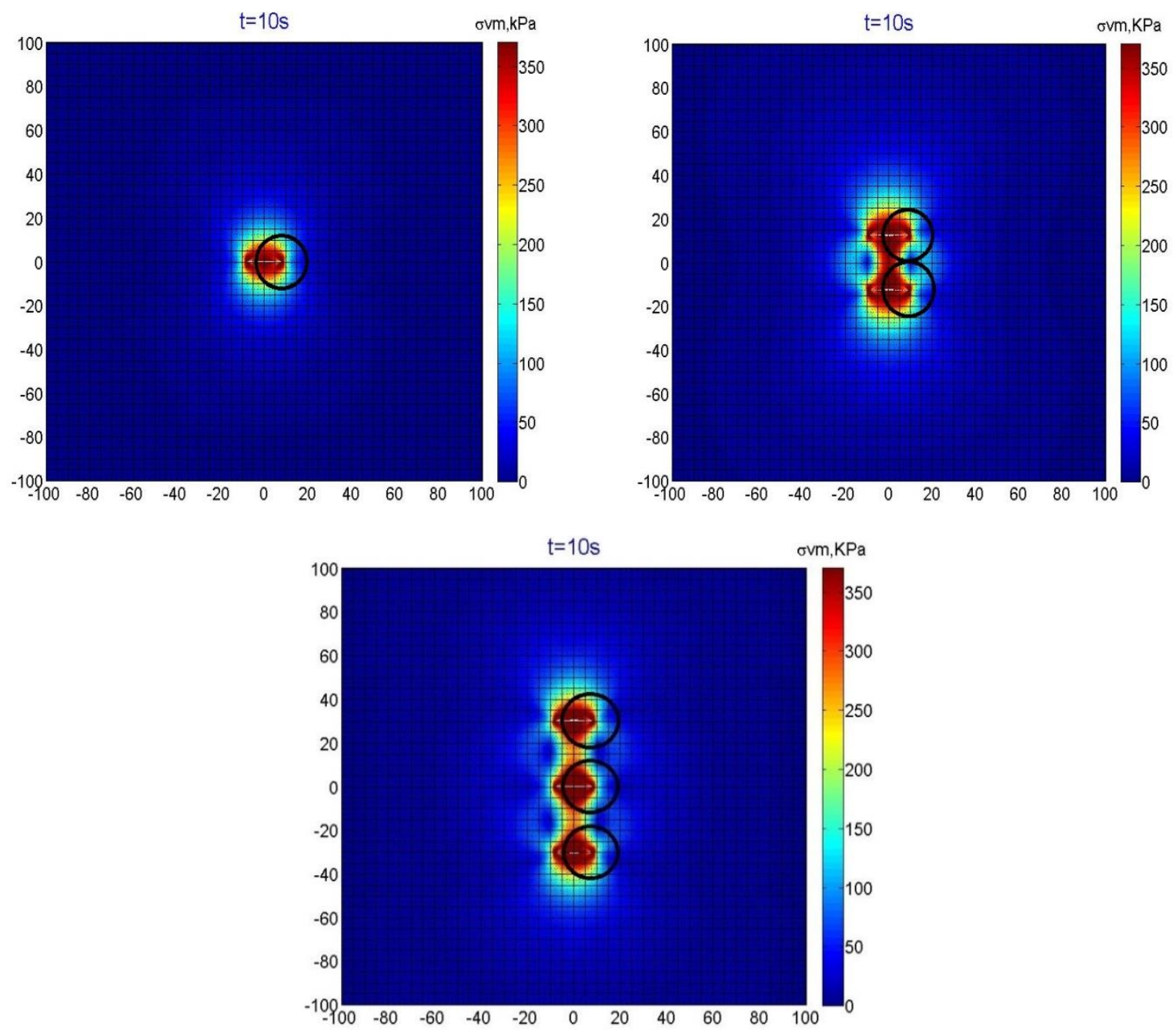

Figure 29 - Critical Radius at the fracture tip of single, two and three fractures after 10s of injection 


\subsection{Mechanical Interactions}

Stress field alterations due to mechanical influence and interaction surrounding fracture play an important role in the development of efficient hydraulic fracturing stages. These interactions control the behavior of current and subsequent fracture development. In a typical hydraulic fracturing study, the stress interactions are often assumed static, aiding in the ease of developing a solution. These assumptions are often too vague and neglecting the time-dependent behavior of fracture propagation, and stress field development can severely hinder the conclusions of the simulation results. Figure 30 illustrates a transient depiction of maximum and minimum principal stresses for three sequential fractures, showing the dynamics of stress behaviors, important in studying mechanical interactions in multi-stage hydraulic fracturing.

In comparison to previous studies of hydraulic fracture stress developments, such as those published by (Cheng, 2009), the static model assumptions indicate a strong weighting towards early time behavior. The early time values depict an agreement in possible conclusions, however, they quickly dissipate throughout the field once the fracture begins to propagate. This provides a fundamental flaw to the static assumptions that are often used throughout the study of stress relating to hydraulic fracture.
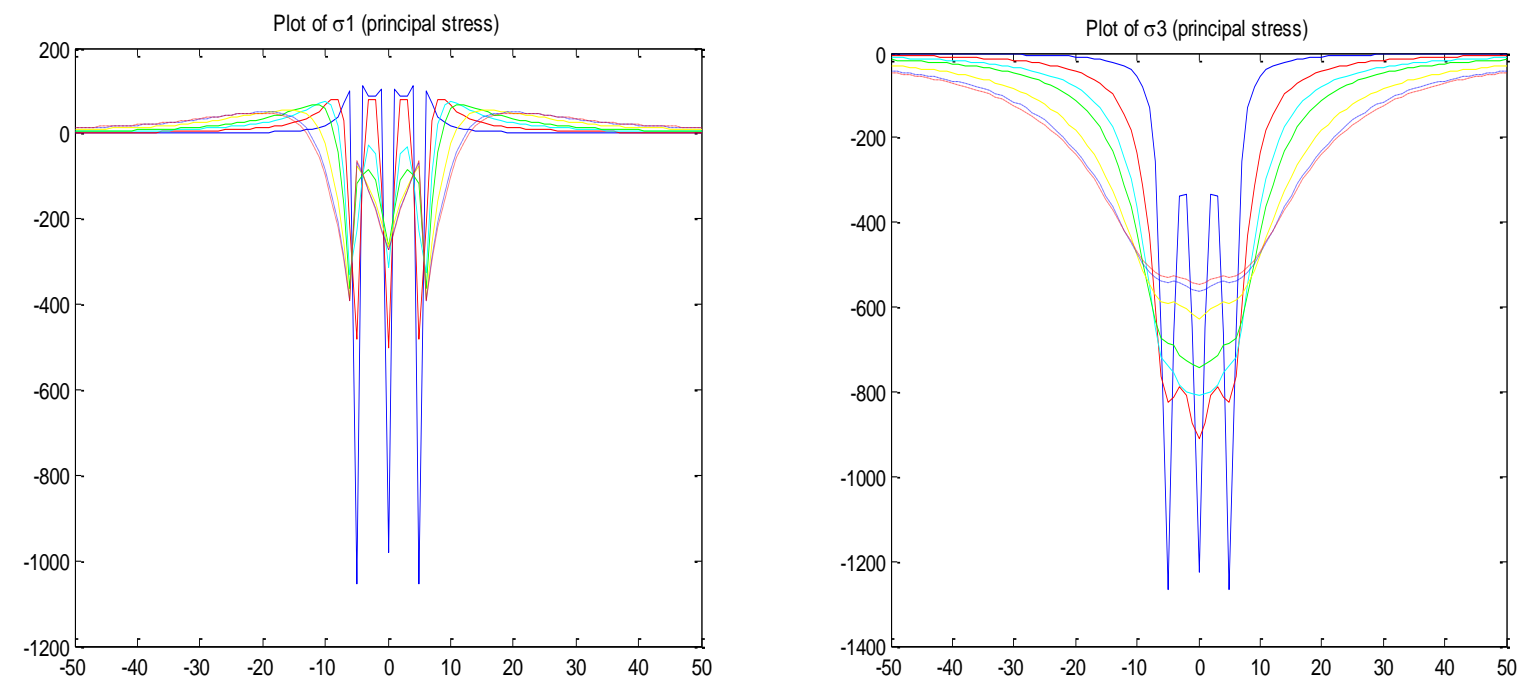

Figure 30 - Development of (a) maximum and (b) minimum principal stresses for three sequential fractures with time

\subsection{Uniform and Non-Uniform Sequential Fracturing}

The uniform injection rate is commonly used to simplify the comparison between fractures and stress fields, i.e., all fractures are equal in both injection rate and volume (Figure 31). Figure 31 shows a change in principal stresses after 40 seconds of simulation. However, creating non-symmetry in the injection rate and observing the interaction of these stresses is important in the optimization of design with sequenced fracture propagation. In Figure 32 the first fracture is receiving the injection rate, which is $40 \%$ of the total specified while the second fracture receives $60 \%$ of the specified mass rate of injection. The application of different injection rate gives us a picture of stress fields surrounding each fracture individually and developing as one whole field. The plot illustrates that stress along the Y-direction 
reducing upon the smaller fracture. The $\mathrm{X}$-direction stresses are displayed as dominant, and they impact the larger fracture stresses.
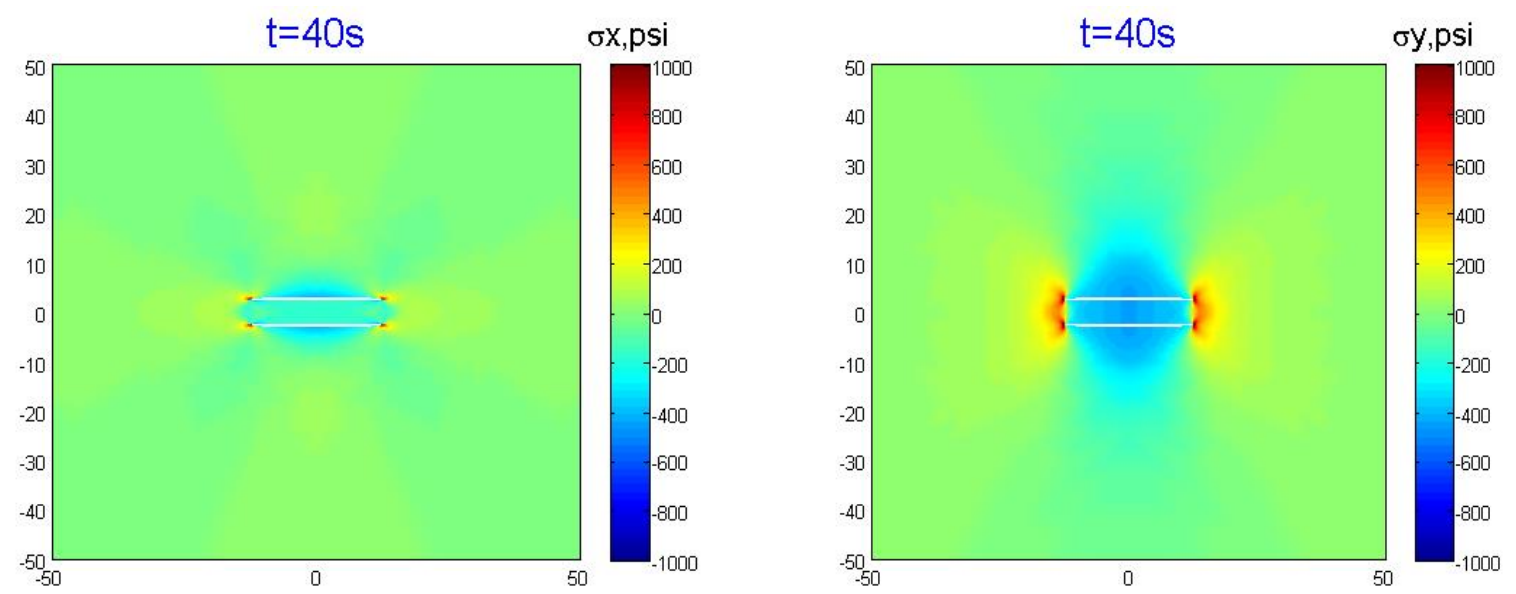

Figure 31 - Sequential fracture propagation under uniform injection rate and a 5-meter spacing
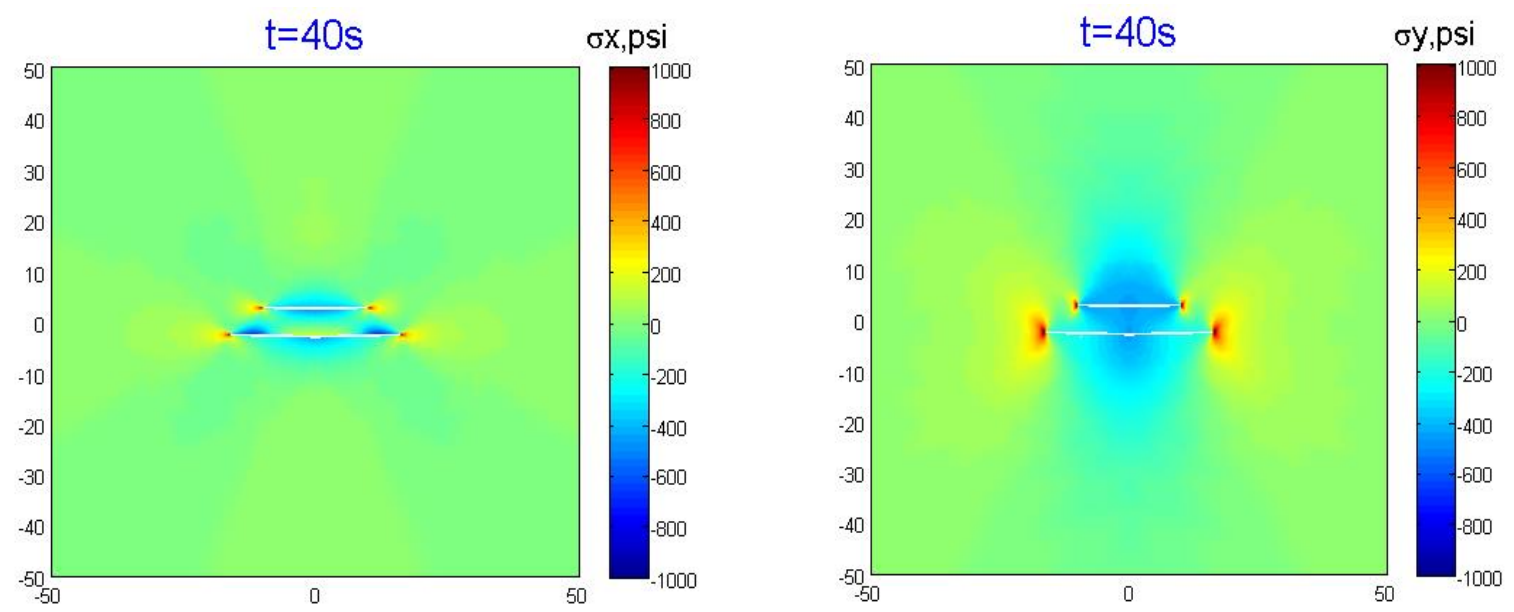

Figure 32 - Sequential fracture propagation under non-uniform injection rate (40/60) and a 5-meter spacing

The evidence of mechanical interactions can be noticed from the change in fracture geometry, as the stresses from one or more fractures alter their length and width. To visualize the interactions of stress fields and their impact on fracture geometry, three sequential fractures under equal injection volume and rate at $5 \mathrm{~m}$ spacing are illustrated in Figure 33. Figure 34 also shows the corresponding Von Mises stress distribution and clear interaction that leads to change in geometry of fractures in Figure 33. To clearly see the interactions of these behaviors, the fracture displacements have been magnified 1000 times. Figure 33 shows three stages: (a) initial fracture propagation stage, when the central fracture is smaller and longer, and it is compressed by the high magnitude of stresses exchanging with the onset of the injection; (b) at later fracture propagation time stresses begin to interact; (c) the impact of the stresses from the central fracture become evident and they cause the expansion of fracture width; (d) at 60 seconds of injection the stress interactions are increased, and the central fracture stays symmetrical while the offset fractures become oblique and the outer edge begins to point inwards. 


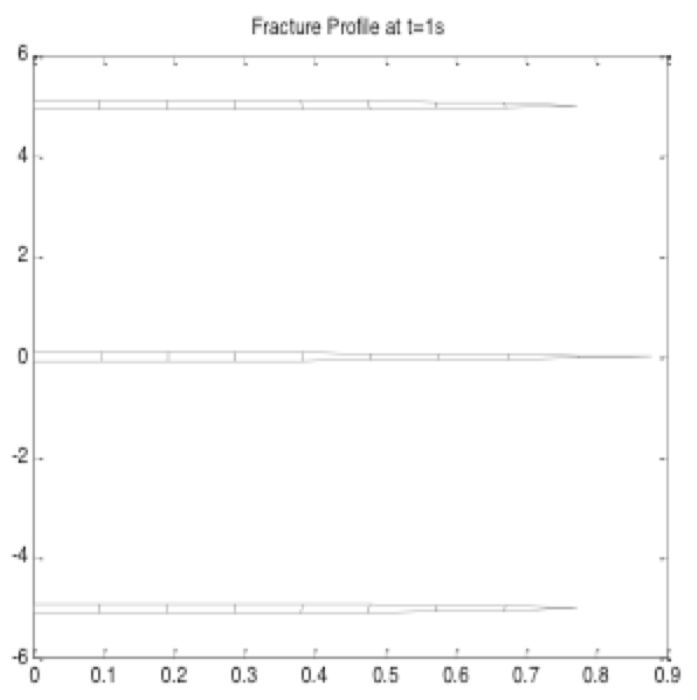

(a)

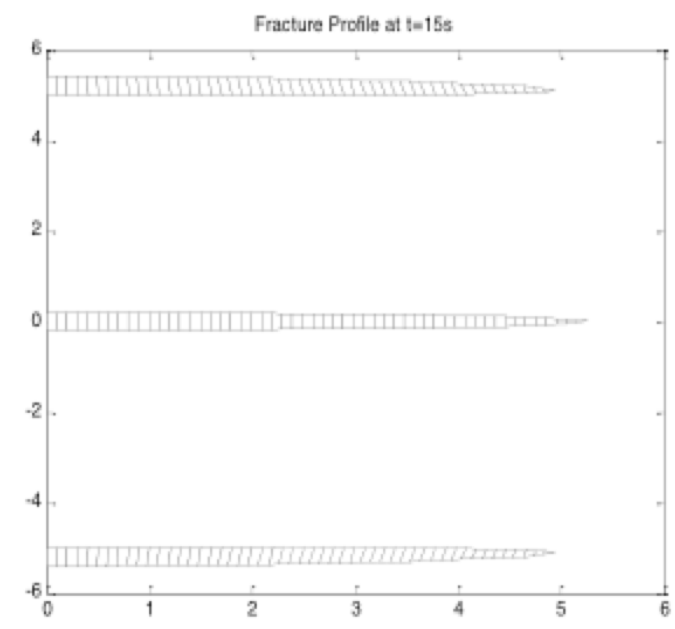

(c)

Figure 33 - Development of fracture half-length geometry of three concurrent fractures at a 5-meter spacing

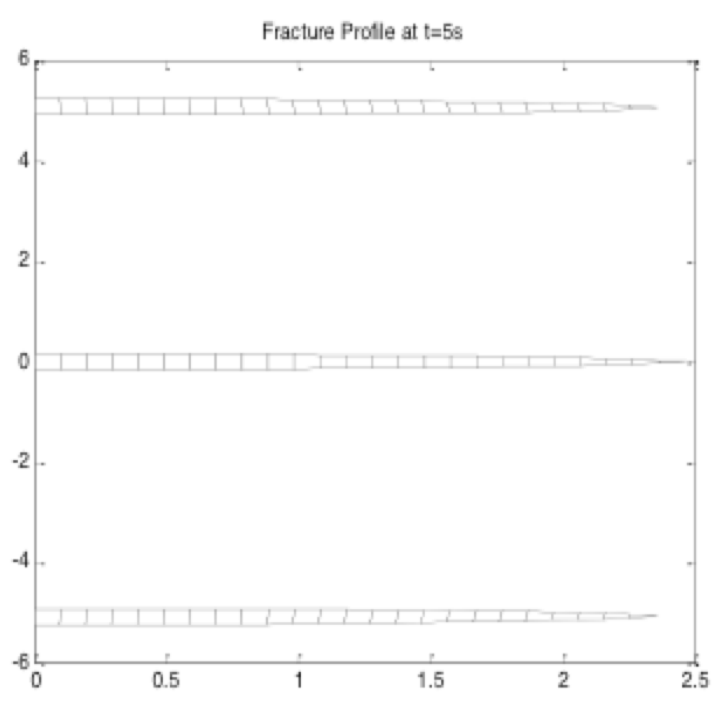

(b)

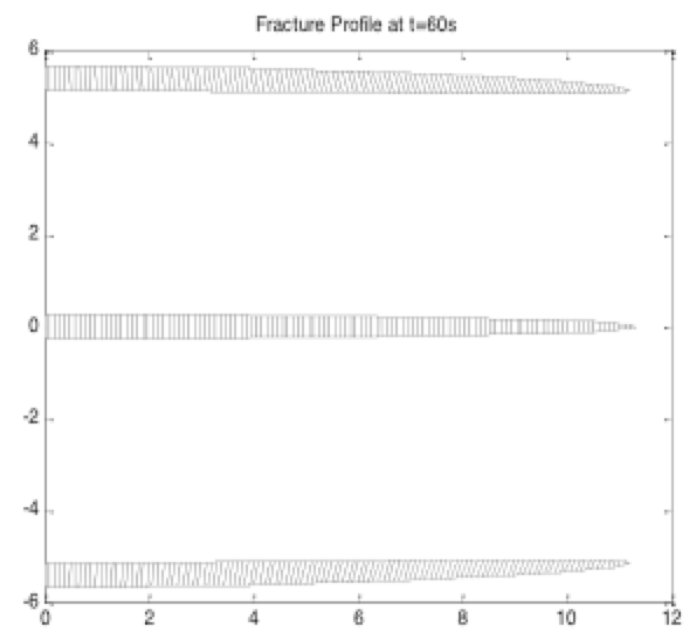

(d)

The concentration of stress plays a significant role in the redistribution of the stress field. Figure 35 displays the comparison of mechanical interaction with respect to a single fracture case. It is apparent that as the spacing between fractures decreases, the fracture geometry deviates from the "ideal" case of what is to be expected in the case of a single fracture. As the fracture spacing is increased - a reduction in the likelihood of interaction is also increased. This process can be inferred through the large differential in the 5 and 10-meter fracture spacing cases while the 25 and 30 - meter cases begin to mirror that of the single fracture. In the case of multi-fracture injection, the mass injection rate is specified as a constant value that is distributed evenly over each fracture. In order to depict the effect of mechanical interactions upon fracture geometry, a single fracture case is used as a baseline. Ensuring each fracture receives an equal amount of energy allows for a comparison of what is known not to be interacting, i.e. single fracture 
case. The single fracture is run using one-third of the injection as that of an otherwise equal three-fracture case, allowing an equivalent comparison between each of the cases to see the influence of the outlying fractures, as both compared fracture cases will be receiving equal amounts of injection. Figure 35 validates the first two best case scenarios of critical radii values $(15.60 \mathrm{~m}$ and $12.64 \mathrm{~m})$, computed with a theory of critical distance (Table 5), and confirms that an optimum fracture spacing has been predicted correctly, and fracture geometry has not been impacted by stress interference and mechanical interactions

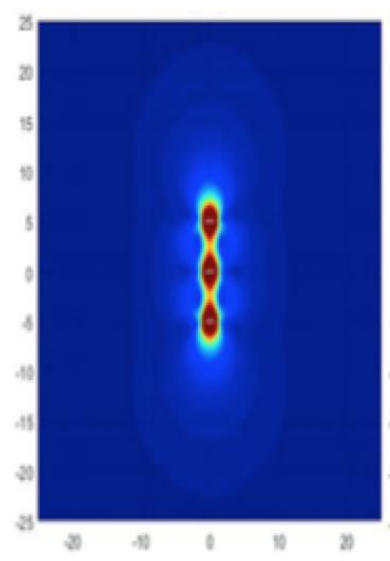

(a)

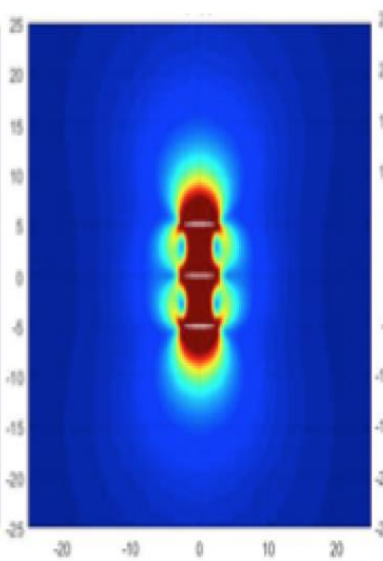

(b)

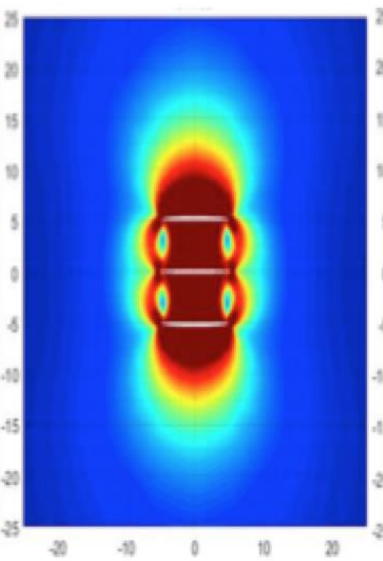

(c)

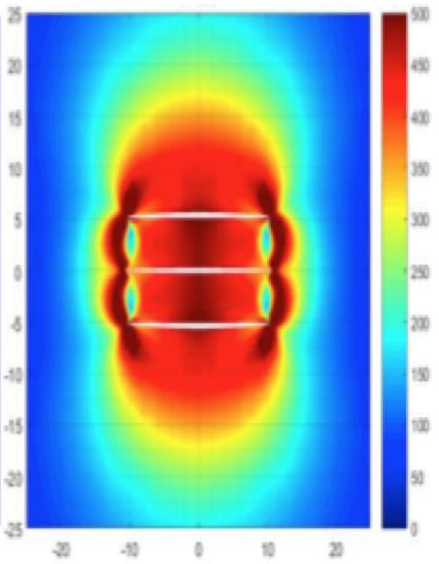

(d)

Figure 34 - Development of the Von Mises stress surrounding three concurrent fractures at a 5-meter spacing

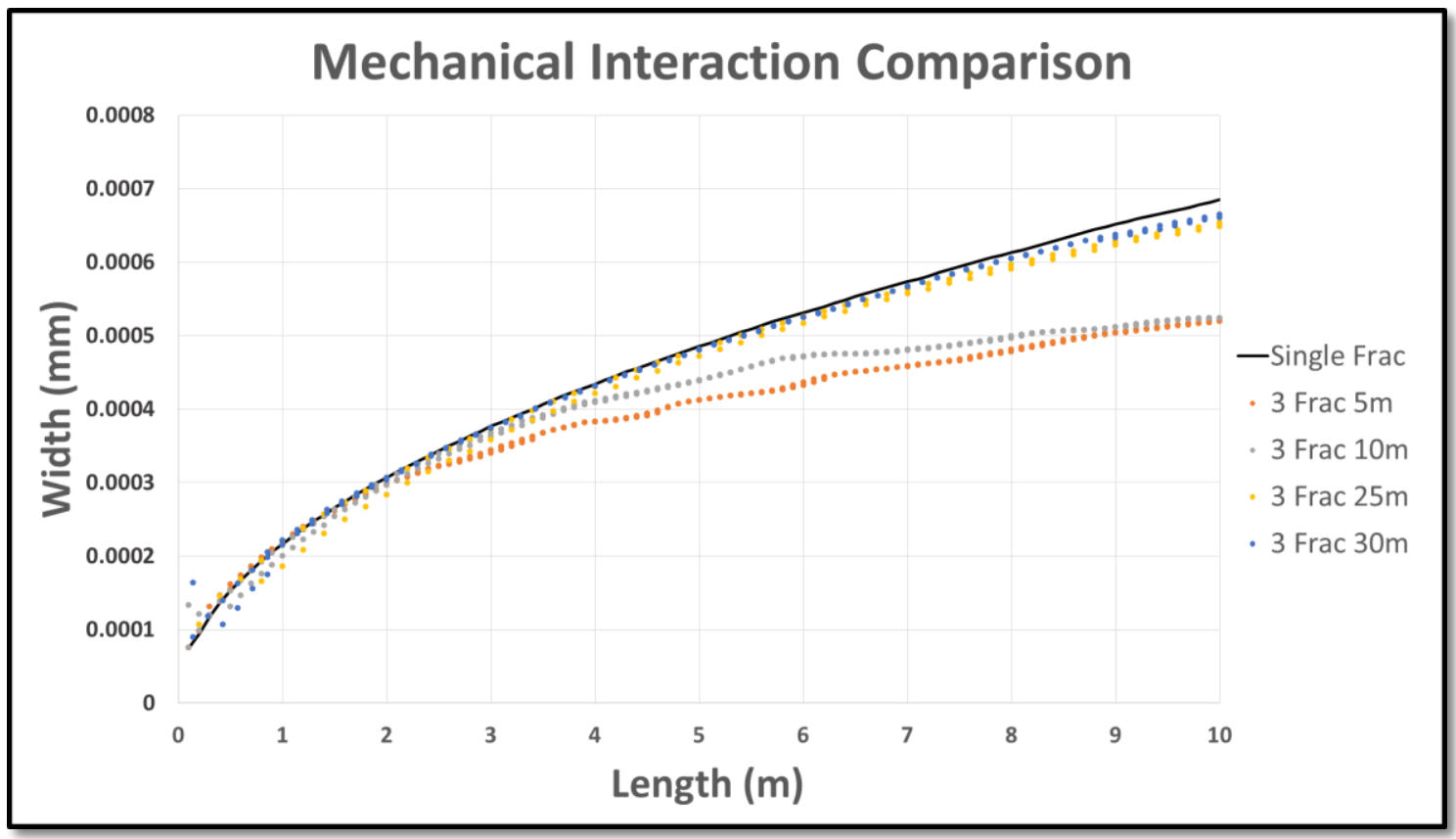

Figure 35 - Display of fracture interaction based upon central fracture 


\subsection{Hydraulic Fracturing in Composite Models}

A composite model was developed in order to compare the stress behavior when hydraulic fracture is crossing the boundary of two layers with different mechanical properties (Figure 36). The domain is comprised of two materials of different modulus of elasticity and Poisson's ratio. The boundary of the second material lies at 10 meters from the point of injection. This will ensure that there is an ample time to see the development of stress as the fracture approaches reaches and passes beyond the layer interface.
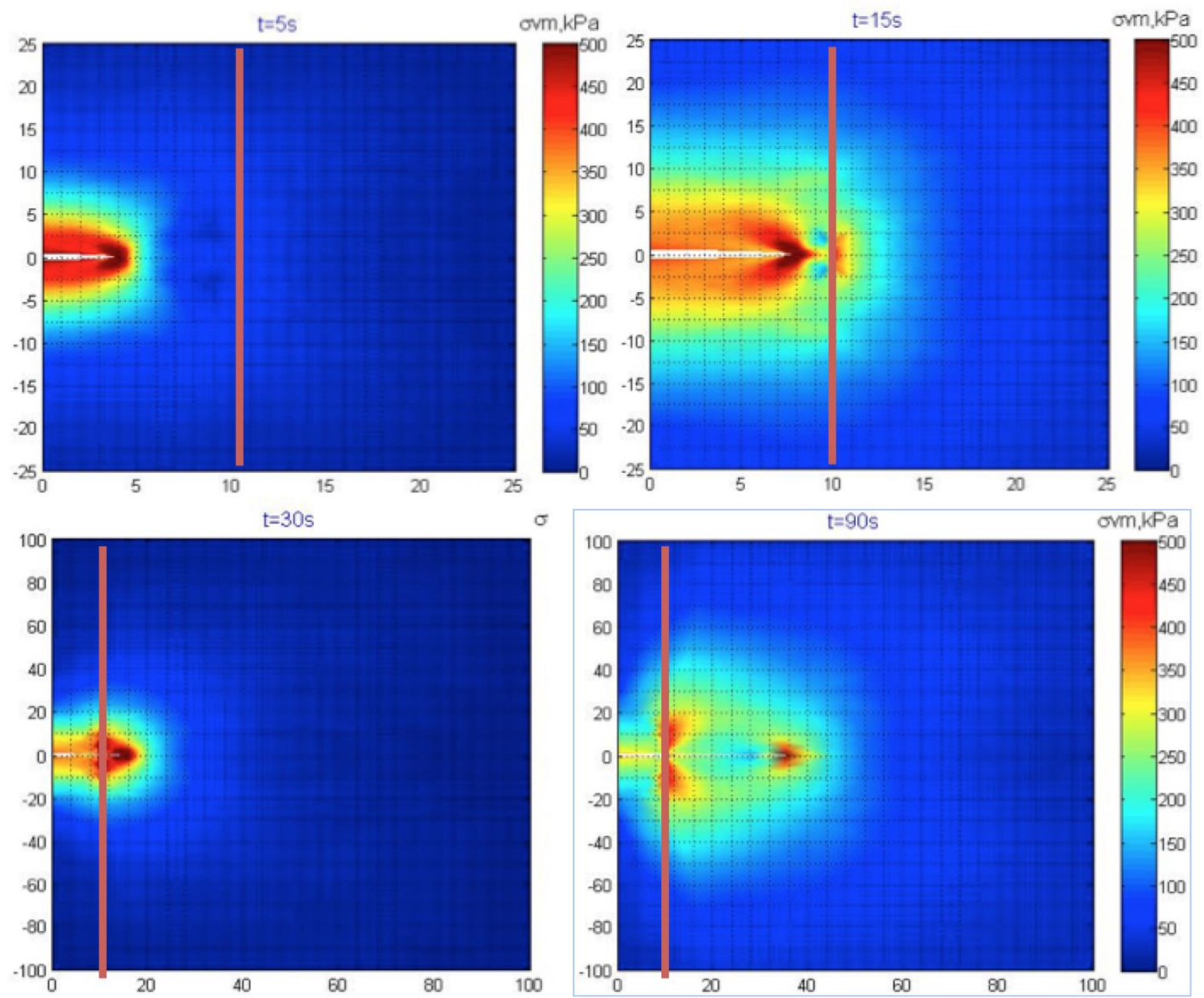

Figure 36 - Von Mises stress distribution throughout fracture propagation in composite model

Table 6 shows details of mechanical properties of each layer for three different cases.

Table 6 - Summary of composite case simulation models

\begin{tabular}{|cll|}
\hline Composite Cases & Modulus of Elasticity & Poisson's Ratio \\
\hline Case 1 & 4.00 to $1.00 \mathrm{GPa}$ & 0.3 to 0.2 \\
Case 2 & $1.77 \mathrm{GPa}$ & 0.2 \\
Case 3 & 1.00 to $4.00 \mathrm{GPa}$ & 0.2 to 0.3 \\
\hline
\end{tabular}


Figure 37 shows the dynamics of Von Mises stress development as single hydraulic fractures getting close to the boundary between two layers reaches the boundary and passing the boundary. As fracture reaches the boundary and passes the boundary both magnitudes of Von Mises stress and fracture geometry significantly being influenced. Figure 37 below depicts the fracture width versus half-length to eliminate any rate dependent comparison between three different cases. The material interface is clearly present at $10 \mathrm{~m}$ where the two component cases display a change in geometry. The first case illustrates a fracture propagating from a harder formation with Young's modulus of $4.00 \mathrm{GPa}$ and Poisson's ratio of 0.3 into a softer formation with Young's modulus of $1.00 \mathrm{GPa}$ and Poisson's ratio of 0.2 . This case displays how a decrease in stiffness occurring across the material interface results in a decrease of energy required for deformation to occur, causing a noticeable jump in fracture width without a change in length over the interface. The third case illustrates the opposite as the fracture is propagating from a softer formation with Young's modulus of $1.00 \mathrm{GPa}$ and Poisson's ratio of 0.2 into a harder formation with Young's modulus of $4.00 \mathrm{GPa}$ and Poisson's ratio of 0.3 . In this case, the fracture undergoes increasing stiffness across the material boundary, and the fracture can be seen to increase in width as the stiffer material is approached, causing a ballooning effect. Once pressure begins to exceed the tensile strength of the rock, the width can be seen to decrease, as the stored energy to break through the stiffer material is enough to regain the original geometry behavior. The second case is given as a base case comparison with no material boundary interface present.

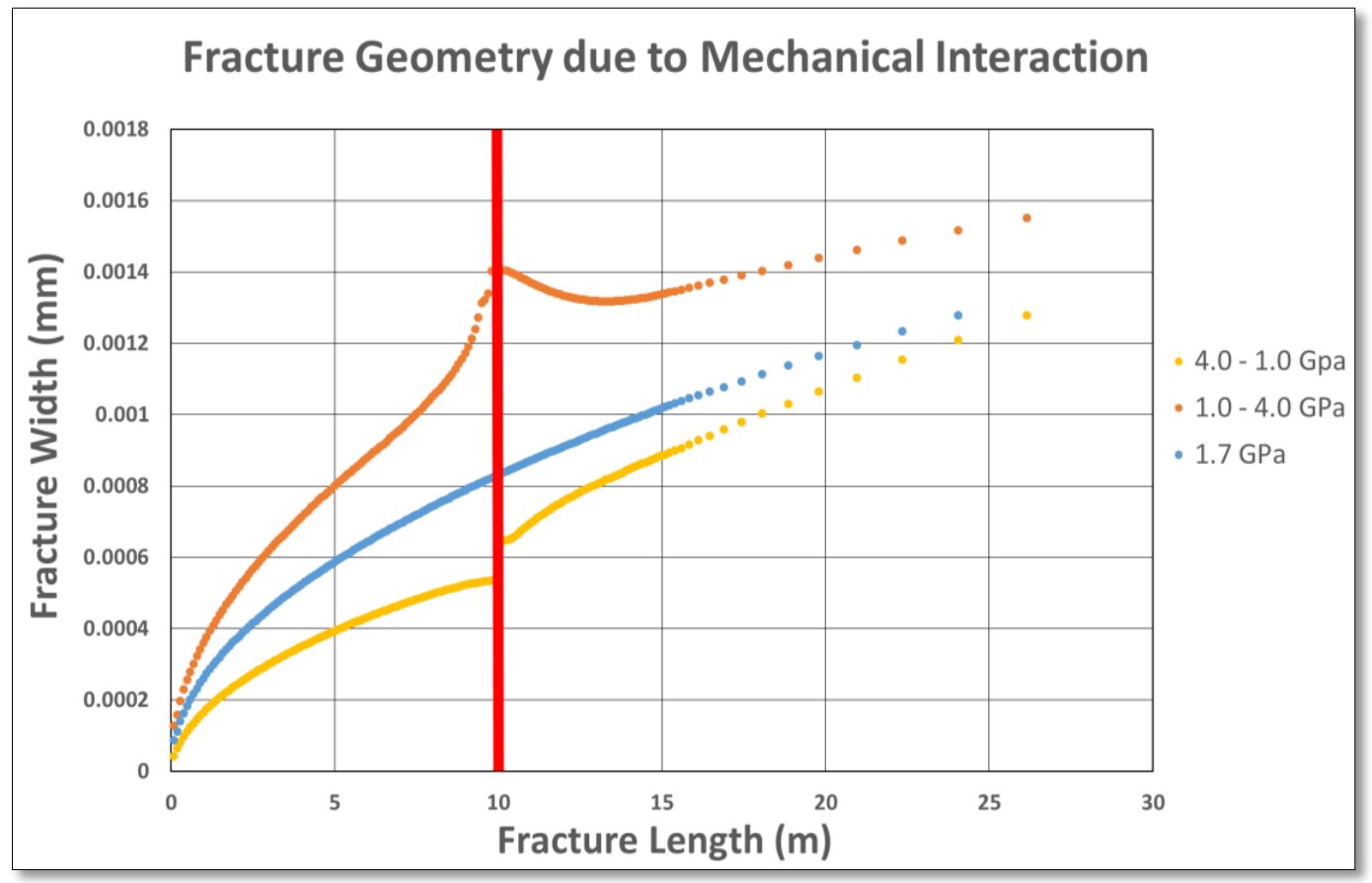

Figure 37 - Fracture geometry due to mechanical interaction in a composite model 


\section{Conclusions}

Modeling fracture network as a function of operation conditions allowed creating dynamic stress profiles during multi-stage hydraulic fracturing and capturing reservoir stress behavior in a real-time. Previous work in this field of study was focused on a static stress behavior. However, there is a visible change in local stress as the fracture propagates and fracture tip becomes distant from the injection point, leaving the reservoir field with significantly dissipating stress values at a later time of fracture propagation. Therefore, models with an assumption of stress remaining static during fracture propagation, don't account for a change in local stress, and they are the main reason of stress interference and fracture merging.

Transient stress profiles, generated from output files of our fully coupled hydromechanical finite element hydraulic fracture model (HFWVU2D, developed under RPSEA, Grant/Contract No: \#9122-06), as well as a further extended in this study theory of critical distance, facilitated a possibility to optimize hydraulic fracture treatment in a real time. This research offered a quantitative analysis of induced stresses and insitu stress reorientation as a function of net fluid pressure on the fracture surface for cases with simultaneous and sequential hydraulic fracturing of a horizontal well for a broad range of hydraulic fracture propagation regimes for both homogenous and composite reservoir models.

The analysis of transient stress patterns from various simulation runs helped identifying the common trend of the stress behavior in the reservoir changing with time. The results clearly show that there is an optimum distance between hydraulic fractures below which, the change (variation) in magnitude and orientation of stresses leads to significant change in fracture geometry and propagation rate. This change has been impacted mainly through mechanical interaction that leads to higher compressive stress concentrations between fractures. The mechanical interaction becomes stronger by increasing the number of fractures or altering the fracture spacing. The effects have also been investigated in a composite reservoir model with different mechanical properties (i.e., Young's modulus and Poisson's ratio), and operational conditions, such as injection rate and volume. Mechanical properties of different layers in a composite reservoir model significantly impact the fracture geometry and propagation rate when the fracture intercepts different layer boundaries. The magnitude of change in stresses and stress reorientation have also been quantified in cross sections with respect to the fracture plane as the fracture propagates.

The prediction of fracture behavior is an integral part of developing a successful stimulation plan. Increasing the understanding of the mechanical interaction, which occurs throughout fracturing stages that can be inferred through working parameters from the field or experimental results, is a goal of this research. The application of modified critical distance theory in combination with numerical simulation results discussed throughout this study highlights a potential for the continued effort and research using such a theory to improve, maximize and optimize fracturing stage efficiency. 


\section{Future Work}

The continuation of this research is valuable both for the energy industry and for the environment. A gradually increasing energy demand requires higher hydrocarbon production rates. Meanwhile, the unconventional shale formations of North America have a tremendous potential to be a leading supplier of energy in the nation, providing energy security and independence for the country. Hydraulic fracturing treatment, applied mostly in unconventional reservoirs, gained success with a proven track record of increased wells productivity, achieved through the increase in reservoir contact area. However, the area of energy technology associated with multi-stage hydraulic fracturing still has a high potential for improvement. Therefore, a better understanding of the reservoir geomechanical response to injected fracturing fluid in a real time allows optimizing hydraulic fracturing treatment and making it more efficient, thus, producing more energy resources for the population while reducing the risk associated with producing hydrocarbons.

My recommendation towards the future direction of this work would be an integration of this dynamic geomechanical model with the fluid flow simulation and the proppant distribution model. In addition, a further improvement of the theory of critical distance for optimizing hydraulic fracturing spacing, and also testing various cases in heterogeneous porous media using real-time experimental data will provide an opportunity to increase the reservoir conductivity significantly. Finally, modifying a node-split

technique for non-planar fracture geometry would allow simulating deviating hydraulic fracture propagation and fracture merging. 


\section{Bibliography}

1. Adachi, J. and Detournay E. (2008). Plain Strain Propagation of a Hydraulic Fracture in a Permeable Rock. pp. 4666-4694.

2. Bao, J.Q, Fathi, E., Ameri, S. (2014). A Coupled Finite Element Method for the Numerical Simulation of Hydraulic Fracturing with a Condensation Technique. Engineering Fracture Mechanics, 269-281.

3. Barton C.A., Zoback M.D. et al. (1995). Fluid flow along potentially active faults in crystaline rock.

4. Broek, D. (1986). Elementary engineering fracture mechanics.

5. Byerlee, J. D. (1978). In Friction of rock (pp. 615-626). Pure \& Applied Geophysics.

6. Carter, B.J., Desroches, J., Ingraffea, A.R., and Wawrzynek, P.A. (2000). Simulating Fully 3D Hydraulic Fracturing. In B. D. Carter, Modeling in Geomechanics 200 (pp. 525-557).

7. Cheng, Y. (2009). Boundary Element Analysis of the Stress Distribution around Multiple Fractures: Implications for the Spacing of Perforation Clusters of Hydraulically Fractured Horizontal Wells. Paper SPE 125769 presented at the SPE Eastern Regional Meeting. Charleston, West Virginia, USA, 23-25 September.

8. Dempsey J. P., A. R. (1999). Scale effect on the in-situ tensile strength and failure of first-year sea ice at Resolute, NWR. International Journal of Fracture, special issue on fracture scaling, 919.

9. Fisher, M.K., Heinze, J.R., Harris, C.D., Davidson, B.M., Wright, C.A., and Dunn, K.P. . (2004). Optimizing Horizontal Completion Technologies in the Barnett Shale Using Microsismic Fracture Mapping. Annual Technical Conference and Exhibition. Houston, Texas: SPE 90051.

10. Fu, P., Johnson, S.M. and Carrigan, C.R. (2012). An Explicitly Coupled Hydro-Geomechanical Model for Simulating Hydraulic Fracturing in Arbitrary Discrete Fracture Networks. Int J Numer Anal Meth Geomech, 278-300.

11. Griffith. (1921). Theory of rupture. Proceedings of the First International Congress of Applied Mechanics, (pp. 55-63).

12. Ito, T. and Hayashi, K. (1991). Physical Bacjground to the Breakdown Pressure in Hydraulic Fracturing Tectonic Stress Measurements. International Journal of Rock Mechanics and Mineral Science and Geomechanics, 285-293.

13. Kong, B. and Fathi, E. (2015). Coupled 3-D Numerical Simulation of Proppant Distribution and Hydraulic Fracturing Performance Optimization in Marcellus Shale Reservoirs. International Journal of Coal Geology, 35-45. 
14. Lajtai, E. (1971). Effect of Tensile Stress Gradient on Brittle Fracture Initiation. Rock Mechanics, 569-578.

15. Louks, R., Askes, H., Susmel, H. (2014). Statis Assesment of Brittle/Ductile Notched Materials: An Engineering Approach Based On the Theory of Critical Distances. Fracture and Structural Integrity related Issues, 23-30.

16. Meyer \& Associates, I. (2015). Meyer Fracturing Simulations. Ninthe Edition.

17. Michael J. Economides, Kenneth G. Nolte. (2000). Reservoir Stimulation. Third Edition.

18. Morrill, J.C. and Miskimins, L. (2012). Optimizing Hydraulic Fracture Spacing in Unconventional Shales. Presented at the SPE Hydraulic Fracturing Technology Conference. Woodlands, Texas, 6-8 February: SPE-152595.

19. Mutalik P.N. and Gibson, B. (2008). Case History of Sequential and Simultaneous Fracturing of the Barnett Shale in Parker County. Paper SPE 116124 presented at the SPE Annual Technical Conference and Exhibition, Denver, 21-24 September.

20. Oltean, R., Taranu, N., Cozmanciuc, C., Banu, C., Ionita, O. (2009). Numerical Methods for the Modeling of Interface Delamination In Composites. Intersections, Vol.6, No.4, 90-98.

21. Perez, N. (2004). Fracture mechanics. New York, Boston, Dordrecht, London, Moscow: Kluwer Academic Publishers.

22. Perkins, T.K. and Kern, L.R. (1961). Width of Hydraulic Fractures. J. Pet. Tech.

23. Roussel, N.P. and Sharma M.M. (2010). Role of Stress Reorientation in the Success of Fracture Treatments in Tight Gas Sands. Paper SPE 134491 presented at the SPE Annual Technical Conference and Exhibition. Florence.

24. Roussel, N.P. and Sharma, M.M. (2011). Optimizing Fracture Spacing and Sequencing in Horizontal-Well Fracturing. SPE Production \& Operations, pp. 173-184.

25. Siebrits, E., Elbel, J.L., Detourney-Piette, C., Christianson, M., Robinson, B.M., and Diyashev, I.R. (1998). Parameters Affecting Azimuth and Length of Secondary Fracture During a Refracture Treatment. Paper SPE 48928 presented at the SPE Annual Technical Conference and Exhibition. New Orleans, 27-30 September.

26. Soliman, M.Y. and Adams, D. (2004). Geo-Mechanics Aspects of Multiple Fracturing of Horizontal and Vertical Wells. Paper SPE 86992 presented at the SPE International Thermal Operations and Heavy Oil Symposium and Western Regional Meeting. Bakersfield, California, USA, 16-18 March.

27. Taylor, D. (2007). The theory of critical distances. A new perspective in fracture mechanics. Oxford, UK: Elsevier.

28. Taylor, D., Kasiri, S., Brazel, E. (2009). The theory of critical distances applied to problems in fracture and fatigue of bone. Dublin: Trinity College. 
29. Warpinski, Abou-Sayed, Moschovidis, Parker. (1993). Hydraulic Fracture Model Comparison Study. Chicago, IL: Gas Research Institute Chicago.

30. Whitney, J.M., R.J. Nuismer. (1974). Composite Materials, 8.

31. Wright C.A., Conant R.A., Golich G.M., Bondor P.L., Murer A.S. and Dobie C.A. (1995). Hydraulic Fracture Orientation and Production/Injection Induced Reservoir Stress Changes in Diatomite Waterfloods. SPE 29625.

32. Yin, T., Tyas, A., Plekhov, O., Terekhina, A., Susmel, L. (2014). On the Use of the Theory of Critical Distances to Estimate the Dynamic Strength of Notched 6063-T5 Aluminum Alloy. Fracture and Structural Integrity related Issues, 220-225.

33. Znong, R., Bao, J. and Fathi, E. (2014). Fully Coupled Finite Element Model to Study Fault Reactivation during Multiple Hydraulic Fracturing in Heterogeneous Tight Formations. Presented at the SPE Eastern Regional Meeting. Charleston, WV, USA, 21-23 October. 